\title{
Two component dark matter with inert Higgs doublet: neutrino mass, high scale validity and collider searches
}

\author{
Subhaditya Bhattacharya, ${ }^{a}$ Purusottam Ghosh, ${ }^{a}$ Abhijit Kumar Saha ${ }^{b}$ \\ and Arunansu $\mathrm{Sil}^{a}$ \\ ${ }^{a}$ Department of Physics, Indian Institute of Technology Guwahati, \\ North Guwahati, Assam- 781039, India \\ ${ }^{b}$ Theoretical Physics Division, Physical Research Laboratory, \\ Ahmedabad 380009, India \\ E-mail: subhab@iitg.ac.in, pghoshiitg@gmail.com, aks@prl.res.in, \\ asil@iitg.ac.in
}

ABSTRACT: The idea of this work is to investigate the constraints on the dark matter (DM) allowed parameter space from high scale validity (absolute stability of Higgs vacuum and perturbativity) in presence of multi particle dark sector and heavy right handed neutrinos to address correct neutrino mass. We illustrate a simple two component DM model, consisting of one inert $\mathrm{SU}(2)_{L}$ scalar doublet and a scalar singlet, both stabilised by additional $\mathcal{Z}_{2} \times \mathcal{Z}_{2}^{\prime}$ symmetry, which also aid to vacuum stability. We demonstrate DM-DM interaction helps achieving a large allowed parameter space for both the DM components by evading direct search bound. High scale validity puts further constraints on the model, for example, on the mass splitting between the charged and neutral component of inert doublet, which has important implication to its leptonic signature(s) at the Large Hadron Collider (LHC).

Keywords: Beyond Standard Model, Higgs Physics, Neutrino Physics

ARXiv EPrint: 1905.12583 


\section{Contents}

1 Introduction 1

2 The model 4

3 Theoretical and experimental constraints $\quad 6$

4 Single component DM frameworks involving $\phi$ or $H^{0} \quad 10$

5 Two component DM set-up with $\phi$ and $H^{0} \quad 12$

$\begin{array}{ll}5.1 \text { Coupled Boltzmann equations and direct search } & 12\end{array}$

$\begin{array}{lll}5.2 & \text { Role of DM-DM conversion in relic density } & 15\end{array}$

6 Relic density and direct search allowed parameter space 18

$\begin{array}{lll}7 & \text { Indirect searches } & 24\end{array}$

8 Electroweak vacuum stability and high scale perturbativity in presence $\begin{array}{lr}\text { of RH neutrino and DM } & \mathbf{2 7}\end{array}$

$\begin{array}{ll}8.1 \text { Neutrino Yukawa couplings } & 27\end{array}$

$\begin{array}{ll}8.2 \beta \text { functions and RG running } & 27\end{array}$

8.3 Allowed parameter space of the model from high scale validity 34

9 Collider signature of inert doublet DM at LHC 36

10 Summary and conclusions $\quad 42$

A Tree level unitarity constraints $\quad 45$

B High scale validity of single component DM models $\quad 47$

C Available parameters of the model from high scale validity for different $\begin{array}{ll}\text { choices of RH neutrino mass and Yukawa coupling } & 48\end{array}$

\section{Introduction}

Discovery of the 'Higgs' boson at Large Hadron Collider (LHC) in 2012 [1, 2] strongly validates the Standard Model (SM) of particle physics as the fundamental governing theory of Strong, Weak and Electromagnetic interactions. However many unresolved issues persist. For example, the electroweak (EW) vacuum turns out to be metastable [3-9] with the present measured value of Higgs mass $\left(m_{h} \sim 125.09 \mathrm{GeV}[10]\right)$ and top quark mass $\left(m_{t} \sim\right.$ $173.1 \mathrm{GeV}[10])$. Large uncertainty in the measured value of $m_{t}$ can even make $\mathrm{EW}$ vacuum 
unstable, questioning the existence of our universe. It is well known that inclusion of additional scalars in the theory can help stabilizing EW vacuum by compensating the negative contributions of fermionic couplings in renormalization group (RG) running of Higgs quartic coupling $\lambda_{H}[11,12]$. This motivates us to look for extended scalar sector.

On the other hand, the existence of dark matter (DM) in the Universe is convincingly supported from the observations of various experiments around the globe, for example, WMAP [13] and Planck [14]. Extensions of SM to accommodate DM is therefore inevitable. The simplest of its kind is Weakly Interacting Massive Particle (WIMP) [15] and most economical is the presence of a singlet scalar dark matter [16-24] with Higgs portal interaction with SM. Non observation of DM in direct search experiments like LUX [25], XENON 1T [25-27], Panda X [28] put a stringent bound on this model (with mass below $1 \mathrm{TeV}$ getting disallowed) due to its prediction of large spin independent (SI) direct search cross section. As an alternative, multi-component DM scenarios [29-48] are proposed where DM-DM interactions (see for example, [39, 40]) play an important role to evade direct search bound.Such an attempt was made in [39], where two singlet scalars serve as two DM components, that satisfy required DM constraints. However, the framework is unable to accommodate the low mass region corresponding to both the DM's masses, simultaneously below $500 \mathrm{GeV}$, to satisfy DM direct search bounds.

Singlet DM fields (even in multipartite framework consisting of two singlet DM candidates [39]) also have limited colliders search possibilities, due to small interaction (Higgs portal) with visible sector. The search strategy for such DM is therefore mainly limited to mono- $X$ signature with missing energy, where $X$ can be jet, $W, Z$ or Higgs [49-51]. Such signal arises out of initial state radiation (ISR) and suffers from huge SM background. Therefore, searching such singlet DM candidates at collider turn out to be difficult, even more due to direct search constraint limiting the DM mass to a higher value. Higher multiplet in dark sector, being equipped with charge components have better possibilities of getting unravelled in future collider search experiments, but tighter constraints arise from dark sector. The simplest of its kind is to assume an inert Higgs $\mathrm{SU}(2)_{L}$ doublet (IDM). However, due to gauge coupling, a single component IDM is severely constrained and is not allowed between DM mass within $80-550 \mathrm{GeV}$, referred as the desert region for under abundance. However, it can produce hadronically quiet single and two lepton signatures with missing energy at LHC $[44,52,53]$. Therefore, it is ideal to study a multipartite DM framework, involving an inert doublet to satisfy DM constraints as well as to have interesting phenomenological consequences.

Physics beyond the SM (BSM) is also strongly motivated by the presence of tiny neutrino mass $(\sim \mathrm{eV})$. In order to explain this tiny neutrino masses, type-I seesaw model is proposed where right handed SM singlet fermions are introduced with their Majorana mass terms. One can then have SM gauge invariant dimension five effective operator of the form $\sim \frac{1}{\Lambda} L L H H$, where $L$ represents SM lepton doublet, $H$ represents SM Higgs doublet and $\Lambda$ is the scale representative of $\mathrm{RH}$ neutrino mass scale. Different seesaw models other than type-I are also invented. They all necessarily predict BSM physics with important and interesting phenomenological consequences. Among these, type-I seesaw is the simplest possibility $[54,55]$, which indicates the presence of heavy right handed $(\mathrm{RH})$ 
neutrinos to yield correct light neutrino mass through additional Yukawa coupling with SM Higgs. This in turn may alter the Higgs vacuum stability condition at high scale (larger than the RH neutrino masses) with large neutrino Yukawa coupling [56-68]. Inclusion of an inert Higgs doublet along with right handed neutrinos can also generate the light neutrino mass radiatively [69]. In that case, the usual neutrino Yukawa coupling involving the SM Higgs doublet can be forbidden with the choice of appropriate discrete symmetry, while a new Yukawa interaction involving the inert Higgs doublet is present. However in such a scenario (with radiative light neutrino mass generation), the EW vacuum stability would not be much different from that of the SM as the running of the Higgs quartic coupling remains unaffected by the presence of this new Yukawa interaction.

Our model under scrutiny addresses some of the interesting features in view of above discussion. We address a multipartite dark sector consisting of a $\mathrm{SU}(2)_{L}$ doublet scalar (the IDM) and a scalar singlet, both stabilized by additional $\mathcal{Z}_{2} \times \mathcal{Z}_{2}^{\prime}$ symmetry (for an earlier effort, see [41]) and provide a two component DM set up. The presence of DMDM interactions enlarge the available parameter space significantly (by reviving the below $500 \mathrm{GeV}$ mass region for both the DM candidates), while the inert DM can also produce leptonic collider signature at LHC. We augment the model with heavy RH neutrinos to address the light neutrino masses. Though the set-up with inert Higgs doublet and $\mathrm{RH}$ neutrinos opens up both the possibilities of generating light neutrinos masses, either through type-I seesaw or via radiative generation, we consider here the neutrino mass generation through type-I seesaw and accordingly choose the appropriate discrete charges of the fields so as to forbid the Yukawa interaction involving SM lepton doublet, RH neutrinos and inert Higgs doublet. The primary reason behind such a choice is to involve the study of the stability of the EW vacuum with large neutrino Yukawa couplings (involving SM Higgs doublet) as mentioned before. Then the parameter space obtained from DM phenomenology will be further constrained from vacuum stability criteria. In brief, we want to study a multipartite DM scenario which would be adversely affected by the nonzero light neutrino mass in terms of EW vacuum stability. Note that while the neutrino Yukawa coupling involving RH neutrinos and SM Higgs tends to destabilze the EW vacuum, the presence of the additional scalars in the set-up tends to stabilize it [56-68]. So, we take up an interesting exercise of validating the model from DM constraints, neutrino masses and high scale validity (absolute stability of the Higgs vacuum and perturbativity). This analysis provides some important conclusions, which are phenomenologically viable at LHC.

Let us finally discuss the plan of the paper. In section 2, we discuss the model construct in details. Section 3 presents possible theoretical and experimental constraints on the model parameters. Then in sections 4,5 and 6 subsequently, we discuss DM phenomenology. Indirect search constraints are discussed in 7 . In section 8 , we investigate the high scale validity of the model. Section 9 summarises collider signature(s) in context of the proposed set up. Finally we conclude in section 10. Tree level unitarity condition is elaborated in appendix A. The high scale stability condition on the single component DM frameworks in presence of RH Neutrino is chalked out in appendix B for the sake of comparison. All the constraints together on the model parameter space along with different choices of RH neutrino mass and Yukawa couplings are listed in two tables in appendix C. 


\begin{tabular}{|c|ccccc|}
\hline BSM and SM Higgs Fields & \multicolumn{5}{|c|}{$\mathrm{SU}(3)_{C} \times \mathrm{SU}(2)_{L} \times \mathrm{U}(1)_{Y} \times \mathcal{Z}_{2} \times \mathcal{Z}_{2}^{\prime} \equiv \mathcal{G}$} \\
\hline$\Phi \equiv\left(\begin{array}{c}H^{+} \\
\frac{1}{\sqrt{2}}\left(H^{0}+i A^{0}\right)\end{array}\right)$ & 1 & 2 & +1 & - & + \\
\hline$\phi$ & 1 & 1 & 0 & + & - \\
\hline$N_{i}(i=1,2,3)$ & 1 & 1 & 0 & + & + \\
\hline$H \equiv\left(\begin{array}{c}w^{+} \\
\frac{1}{\sqrt{2}}(h+v+i z)\end{array}\right)$ & 1 & 2 & +1 & + & + \\
\hline
\end{tabular}

Table 1. Charge assignments of the BSM fields assumed in the model under $\mathcal{G}$ as well as that of SM Higgs. The $\mathrm{U}(1)_{Y}$ hypercharge is chosen as $Q=T_{3}+Y / 2$.

\section{The model}

The model is intended to capture the phenomenology of two already established DM frameworks involving that of a singlet scalar and that of an inert scalar doublet together with right handed neutrinos to address neutrino mass under the same umbrella. Therefore, we extend SM by an inert doublet scalar $(\Phi)$ and a real scalar singlet $(\phi)$ and include three RH Majorona neutrinos $N_{i}(i=1,2,3)$ in the set up. The lightest neutral scalar mode of the IDM and $\phi$ are the DM candidates provided an appropriate symmetry in addition to that of SM stabilizes both of them. This is minimally ${ }^{1}$ possible by introducing an additional $\mathcal{Z}_{2} \times \mathcal{Z}_{2}^{\prime}$ discrete symmetry under which all SM fields along with the right handed neutrinos transform trivially and the other additional fields transform non-trivially as tabulated in the table 1. We also note the charges of SM Higgs $(H)$ explicitly in table 1 , as it will be required to form the scalar potential of the model. Note here, that charges of the two DM candidates $(\Phi$ and $\phi)$ are complementary, i.e. odd under either $\mathcal{Z}_{2}$ or $\mathcal{Z}_{2}^{\prime}$ for their stability. We also point out that the $\mathrm{U}(1)_{Y}$ hypercharge assignment of $\Phi$ is identical to SM doublet $H$. Therefore the only $\mathrm{SU}(2)_{L} \times \mathrm{U}(1)_{Y}$ invariant terms are $H^{\dagger} H, \Phi^{\dagger} \Phi, H^{\dagger} \Phi$ and its conjugate.

The scalar Lagrangian reads as:

$$
\begin{aligned}
\mathcal{L}_{\text {scalar }} & =\left|D^{\mu} H\right|^{2}+\left|D^{\mu} \Phi\right|^{2}+\frac{1}{2}\left(\partial^{\mu} \phi\right)^{2}-V(H, \Phi, \phi), \\
\text { where } D^{\mu} & =\partial^{\mu}-i g_{2} \frac{\sigma^{a}}{2} W^{a \mu}-i g_{1} \frac{Y}{2} B^{\mu}
\end{aligned}
$$

and $g_{2}, g_{1}$ denote $\mathrm{SU}(2)_{L}$ and $\mathrm{U}(1)_{Y}$ coupling respectively.

The most relevant renormalizable scalar potential in this case is given by,

$$
V(H, \Phi, \phi)=-\mu_{H}^{2}\left(H^{\dagger} H\right)+\lambda_{H}\left(H^{\dagger} H\right)^{2}+V(H, \Phi)+V(H, \phi)+V(\Phi, \phi)
$$

\footnotetext{
${ }^{1}$ Introduction of a single $\mathcal{Z}_{2}$ could lead to a decay of one of the DM candidates, as seen from an allowed term $\Phi^{\dagger} H \phi$ in that case.
} 


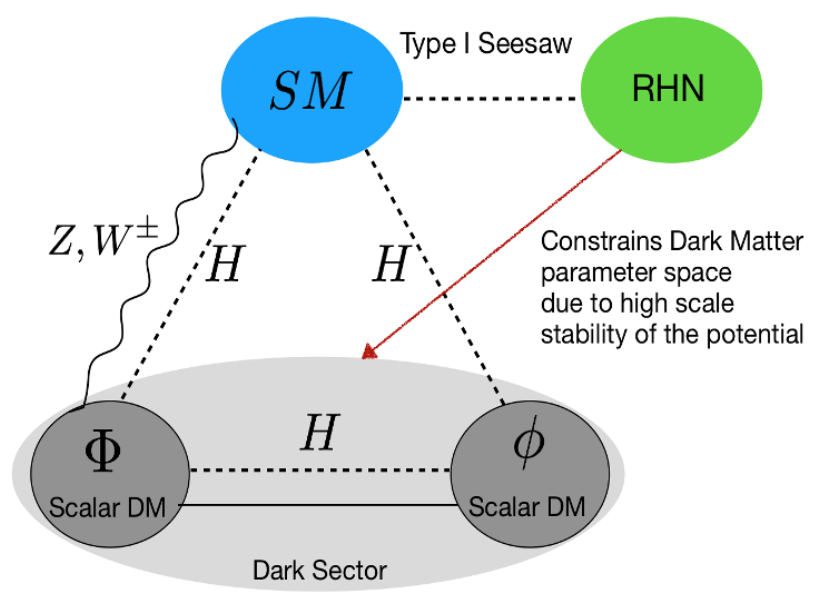

Figure 1. A schematic diagram illustrating the different sectors of the model and their connection to SM. The dotted lines represent Higgs portal coupling, wavy line indicate gauge coupling, while the thin solid line indicates direct DM-DM coupling through $\frac{\lambda_{c}}{2} \phi^{2}\left(\Phi^{\dagger} \Phi\right)$ term.

where,

$$
\begin{aligned}
V(H, \Phi)= & \mu_{\Phi}^{2}\left(\Phi^{\dagger} \Phi\right)+\lambda_{\Phi}\left(\Phi^{\dagger} \Phi\right)^{2}+\lambda_{1}\left(H^{\dagger} H\right)\left(\Phi^{\dagger} \Phi\right) \\
& +\lambda_{2}\left(H^{\dagger} \Phi\right)\left(\Phi^{\dagger} H\right)+\frac{\lambda_{3}}{2}\left[\left(H^{\dagger} \Phi\right)^{2}+\text { h.c. }\right], \\
V(H, \phi)= & \frac{1}{2} \mu_{\phi}^{2} \phi^{2}+\frac{\lambda_{\phi}}{4 !} \phi^{4}+\frac{1}{2} \lambda_{\phi h} \phi^{2}\left(H^{\dagger} H\right) \\
V(\Phi, \phi)= & \frac{\lambda_{c}}{2}\left(\phi^{2}\right)\left(\Phi^{\dagger} \Phi\right) .
\end{aligned}
$$

The Lagrangian involving right handed neutrinos can be written as,

$$
\mathcal{L}^{\nu}=-\left(Y_{\nu}\right)_{i j} \bar{l}_{L_{i}} \widetilde{H} N_{j}-\frac{1}{2} M_{N_{i j}} \overline{N_{i}^{C}} N_{j}
$$

where $\tilde{H}=i \sigma_{2} H^{*}$. We have considered three generations of RH neutrinos with $\{i, j\}=$ $1,2,3$, which can acquire Majorana masses and can possess Yukawa interactions with SM lepton doublet $l_{L}$. Note here, that the charge assignment of the $N$ fields then aid us to obtain neutrino masses through standard Seesaw-I $[54,55]$ mechanism (as detailed later), while it also prohibits the operator like $\overline{l_{L}} \Phi N$ due to $\mathcal{Z}_{2}$ charge assignment, and hence discards the possibility of generating the light neutrino mass radiatively. The ingredients and interactions of the model set up is described in the cartoon as in figure 1.

After spontaneous symmetry breaking, SM Higgs doublet acquires non-zero vacuum expectation value $(\mathrm{VEV})$ as $H=\left(0 \frac{v+h}{\sqrt{2}}\right)^{T}$ with $v=246 \mathrm{GeV}$. Also note that neither of the added scalars acquire VEV to preserve $\mathcal{Z}_{2} \times \mathcal{Z}^{\prime}{ }_{2}$ and act as DM components. After minimizing the potential $V(H, \Phi, \phi)$ along different field directions, one can obtain the 
following relations between the physical masses and the couplings involved:

$$
\begin{aligned}
& \mu_{H}^{2}=\frac{m_{h}^{2}}{2}, \quad \mu_{\Phi}^{2}=m_{H^{0}}^{2}-\lambda_{L} v^{2}, \quad \lambda_{3}=\frac{1}{v^{2}}\left(m_{H^{0}}^{2}-m_{A^{0}}^{2}\right), \\
& \lambda_{2}=\frac{1}{v^{2}}\left(m_{H^{0}}^{2}+m_{A^{0}}^{2}-2 m_{H^{ \pm}}^{2}\right) \quad \text { and } \quad \lambda_{1}=2 \lambda_{L}-\frac{2}{v^{2}}\left(m_{H^{0}}^{2}-m_{H^{ \pm}}^{2}\right),
\end{aligned}
$$

where $\lambda_{L}=\frac{1}{2}\left(\lambda_{1}+\lambda_{2}+\lambda_{3}\right)$ and $m_{h}, m_{H^{0}}, m_{A^{0}}$ are the mass eigenvalues of SM-like neutral scalar found at LHC $\left(m_{h}=125.09 \mathrm{GeV}\right)$, heavy or light additional neutral scalar and the CP-odd neutral scalar respectively. $m_{H^{ \pm}}$denotes the mass of charged scalar eigenstate(s). The mass for $\phi$ DM will be rescaled as $m_{\phi}^{2}=\mu_{\phi}^{2}+\frac{1}{2} \lambda_{\phi h} v^{2}$. The independent parameters of the model, those are used to evaluate the DM, neutrino mass constraints are as follows:

$$
\text { Parameters : } \quad\left\{m_{H^{0}}, m_{A^{0}}, m_{H^{ \pm}}, m_{\phi}, \lambda_{L}, \lambda_{\phi h}, \lambda_{\Phi}, \lambda_{\phi}, Y_{\nu_{i j}}, M_{N_{i j}}\right\} .
$$

\section{Theoretical and experimental constraints}

We would like to address possible theoretical and experimental constraints on model parameters here.

Stability. In oder to get the potential bounded from below, the quartic couplings of the potential $V(H, \Phi, \phi)$ must have to satisfy following co-positivity conditions (CPC) as given by $[70,71]$,

$$
\begin{aligned}
\mathrm{CPC}\{1,2,3\}: & \lambda_{H}(\mu) \geq 0, \quad \lambda_{\Phi}(\mu) \geq 0, \quad \lambda_{\phi}(\mu) \geq 0, \\
\mathrm{CPC}\{4,5\}: & \left(\lambda_{1}(\mu)+\lambda_{2}(\mu) \pm \lambda_{3}(\mu)\right)+\sqrt{\lambda_{H}(\mu) \lambda_{\Phi}(\mu)} \geq 0, \\
\mathrm{CPC}\{6,7\}: & \lambda_{1}(\mu)+2 \sqrt{\lambda_{H}(\mu) \lambda_{\Phi}(\mu)} \geq 0, \quad \lambda_{\phi h}(\mu)+\sqrt{\frac{2}{3} \lambda_{H}(\mu) \lambda_{\Phi}(\mu)} \geq 0, \\
\mathrm{CPC} 8: & \lambda_{c}(\mu)+\sqrt{\frac{2}{3} \lambda_{\Phi}(\mu) \lambda_{\phi}(\mu)} \geq 0,
\end{aligned}
$$

where $\operatorname{CPC}\{i\}$ denotes $i^{\text {th }}$ copositivity condition and $\mu$ is the running scale. The above conditions show that the model offers to choose even negative $\lambda_{1,2,3, \phi h}$ satisfying the above conditions. However, as demonstrated in eq. (2.8), we use $\lambda_{L}$ and physical masses to be the parameters. Therefore, if we choose a specific mass hierarchy as: $m_{H^{ \pm}} \geq m_{A^{0}} \geq m_{H^{0}}$ with positive $\lambda_{L}$, we are actually using $\lambda_{1}$ to be positive while $\lambda_{2,3}$ negative abiding by the above conditions (see eq. (2.7)). The conditions $\operatorname{CPC}\{i\}$ as in eq. (3.1), will be used later in demonstrating stability of the potential in section 8 .

Perturbativity. In oder to maintain perturbativity, the quartic couplings of the scalar potential $V(H, \Phi, \phi)$, gauge couplings $\left(g_{i=1,2,3}\right)$ and neutrino Yukawa coupling $Y_{\nu}$ should obey:

$$
\begin{aligned}
& \left|\lambda_{H}(\mu)\right|<4 \pi, \quad\left|\lambda_{\Phi}(\mu)\right|<4 \pi, \quad\left|\lambda_{\phi}(\mu)\right|<4 \pi, \\
& \left|\lambda_{c}(\mu)\right|<4 \pi, \quad\left|\lambda_{\phi h}(\mu)\right|<4 \pi, \quad\left|\lambda_{1}(\mu)\right|<4 \pi, \\
& \left|\lambda_{2}(\mu)\right|<4 \pi, \quad\left|\lambda_{3}(\mu)\right|<4 \pi, \quad\left|g_{i=1,2,3}\right|<\sqrt{4 \pi} \\
& \text { and } \quad\left|\operatorname{Tr}\left[Y_{\nu}^{\dagger}(\mu) Y_{\nu}(\mu)\right]\right|<4 \pi \text {. }
\end{aligned}
$$



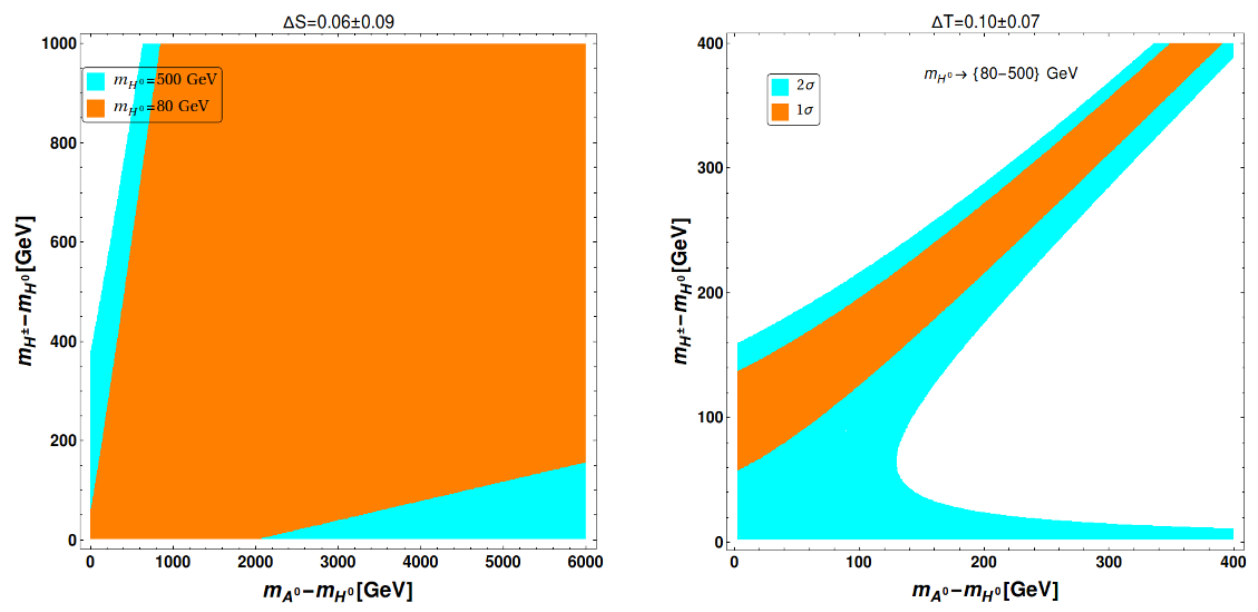

Figure 2. Constraints from $\Delta S$ (left) and $\Delta T$ (right) in $m_{A^{0}}-m_{H^{0}}$ and $m_{H^{ \pm}}-m_{H^{0}}$ plane. For $\Delta S$, we have taken $1 \sigma$ limit for two different choices of $m_{H^{0}}=\{80,500\} \mathrm{GeV}$. For $\Delta T$ scan, we show both $1 \sigma$ and $2 \sigma$ limits for a range of $m_{H^{0}}=\{80-500\} \mathrm{GeV}$.

Tree level unitarity. Next we turn to the constraints imposed by tree level unitarity of the theory, coming from all possible $2 \rightarrow 2$ scattering amplitudes as detailed in appendix A follows as $[72,73]$ :

$$
\begin{array}{rlrl}
\left|\lambda_{H}\right| & <4 \pi, & \left|\lambda_{\Phi}\right| & <4 \pi, \\
\left|\lambda_{c}\right| & <8 \pi, & \left|\lambda_{\phi h}\right| & <8 \pi, \\
\left|\lambda_{1}\right| & <8 \pi, & \left|\lambda_{1}+2\left(\lambda_{2}+\lambda_{3}\right)\right| & <8 \pi \\
\left|\lambda_{1}-\lambda_{2}-\lambda_{3}\right| & <8 \pi, \\
\left|\lambda_{1}+\lambda_{2}+\lambda_{3}\right| & <8 \pi, & \left|\left(\lambda_{\Phi}+\lambda_{H}\right) \pm \sqrt{\left(\lambda_{2}+\lambda_{3}\right)^{2}+\left(\lambda_{H}-\lambda_{\Phi}\right)^{2}}\right| & <8 \pi, \\
\text { and }\left|x_{1,2,3}\right| & <16 \pi,
\end{array}
$$

where $x_{1,2,3}$ be the roots of the cubic equation as detailed in appendix A.

Electroweak precision parameters. There exists an additional $\mathrm{SU}(2)_{L}$ doublet $(\Phi)$ in our model in addition to a gauge singlet scalar $(\phi)$.

As the vev of $\Phi$ is zero, it does not alter the SM predictions of electroweak $\rho$ parameter [74]. However IDM, being an $\mathrm{SU}(2)_{L}$ doublet makes a decent contribution to $S$ and $T$ parameters $[75,76]$ which we will identify as $\Delta S$ and $\Delta T$. The experimental bound from the global electroweak fit results on $\Delta S$ and $\Delta T$ using $\Delta U=0$ are given by:

$$
\left.\Delta S\right|_{\Delta U=0}=0.06 \pm 0.09,\left.\quad \Delta T\right|_{\Delta U=0}=0.1 \pm 0.07,
$$

at $1 \sigma$ level with correlation coefficient 0.91 [77].

We show the constraint from $\Delta S$ and $\Delta T$ on the model parameter space in figure 2 using the standard formula as presented in $[75,76]$. In left plot, we scan $1 \sigma$ fluctuation on $\Delta S$ in $m_{A^{0}}-m_{H^{0}}$ versus $m_{H^{ \pm}}-m_{H^{0}}$ plane for two different values of $m_{H^{0}}=\{80,500\} \mathrm{GeV}$. We see that for smaller $m_{H^{0}}$, the constraint is larger. In right panel, we show $1 \sigma$ and 
$2 \sigma$ limits from $\Delta T$ in $m_{A^{0}}-m_{H^{0}}$ versus $m_{H^{ \pm}}-m_{H^{0}}$ plane for a range of IDM mass $m_{H^{0}}=\{80-500\} \mathrm{GeV}$. We can clearly see, that $\Delta T$ constrains the mass splitting much more than $\Delta S$.

Higgs invisible decay. Whenever the DM particles are lighter than half of the SM Higgs mass, the Higgs can decay to DM and therefore it will contribute to Higgs invisible decay. Therefore, in such circumstances, we have to employ the bound on the invisible decay width of the $125.09 \mathrm{GeV}$ Higgs as [10]:

$$
\begin{aligned}
\operatorname{Br}(h \rightarrow \mathrm{Inv}) & <0.24 \\
\frac{\Gamma(h \rightarrow \mathrm{Inv})}{\Gamma(h \rightarrow \mathrm{SM})+\Gamma(h \rightarrow \mathrm{Inv})} & <0.24
\end{aligned}
$$

where

$$
\Gamma(h \rightarrow \operatorname{Inv})=\Gamma\left(h \rightarrow H^{0} H^{0}\right)+\Gamma(h \rightarrow \phi \phi), \text { when } m_{\phi}, m_{H_{0}}<m_{h} / 2 \sim 62.5 \mathrm{GeV} ;
$$

and $\Gamma(h \rightarrow \mathrm{SM})=4.2 \mathrm{MeV}[10]$. In this analysis, we have mostly focused in the region where $m_{\phi}, m_{H_{0}}>m_{h} / 2$, actually larger than $W$ mass, i.e. $m_{\phi}, m_{H_{0}} \geq m_{W}$, so that the above constraint is not applicable.

Collider search constraints. Experimental searches for additional charged scalars and pseudoscalar in LEP and LHC provide bound on IDM mass parameters and coupling coefficients of IDM with SM particles.

(i) Bounds from LEP. The observed decay widths of $Z$ and $W$ bosons from LEP data restrict the decay of gauge bosons to the additional scalars and therefore provide a bound on IDM mass parameters as $m_{A^{0}}+m_{H^{0}}>m_{Z}, 2 m_{H^{ \pm}}>m_{Z}$ and $m_{H^{ \pm}}+$ $m_{H^{0}, A^{0}}>m_{W}$. In addition, neutralino searches at LEP-II, provides a lower limit on the pseudoscalar Higgs $\left(m_{A^{0}}\right)$ to $100 \mathrm{GeV}$ when $m_{H^{0}}<m_{A^{0}}$ [78]. The chargino search at LEP-II limits indicate a bound on the charged Higgs to $m_{H^{ \pm}}>70 \mathrm{GeV}$ [79].

(ii) Bounds from LHC. Due to the presence of SM Higgs and IDM interaction, charged scalars $H^{ \pm}$take part into the decay of SM Higgs to diphoton. Thus it contributes to Higgs to diphoton signal strength $\mu_{\gamma \gamma}$ which is defined as [80-83]

$$
\mu_{\gamma \gamma}=\frac{\sigma(g g \rightarrow h \rightarrow \gamma \gamma)}{\sigma(g g \rightarrow h \rightarrow \gamma \gamma)_{\mathrm{SM}}} \simeq \frac{\operatorname{Br}(h \rightarrow \gamma \gamma)_{\mathrm{IDM}}}{\operatorname{Br}(h \rightarrow \gamma \gamma)_{\mathrm{SM}}}
$$

Now when IDM particles are heavier than $m_{h} / 2$, one can further write

$$
\frac{\operatorname{Br}(h \rightarrow \gamma \gamma)_{\mathrm{IDM}}}{\operatorname{Br}(h \rightarrow \gamma \gamma)_{\mathrm{SM}}}=\frac{\Gamma(h \rightarrow \gamma \gamma)_{\mathrm{IDM}}}{\Gamma(h \rightarrow \gamma \gamma)_{\mathrm{SM}}}
$$

The analytic expression of $\Gamma(h \rightarrow \gamma \gamma)_{\mathrm{IDM}}$ can be obtained as [80-83]:

$$
\Gamma(h \rightarrow \gamma \gamma)_{\mathrm{IDM}}=\left|\mathcal{A}_{\mathrm{SM}}+\frac{\alpha_{e} m_{h}^{3 / 2}}{16 \pi^{3 / 2}} \frac{\lambda_{1} v}{m_{H^{ \pm}}^{2}} F\left(\frac{m_{h}^{2}}{4 m_{H^{ \pm}}^{2}}\right)\right|^{2},
$$




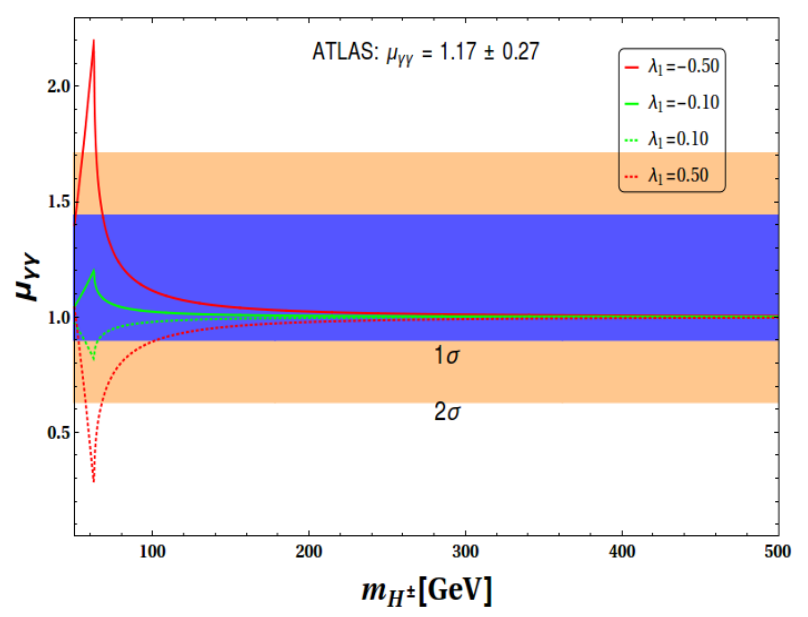

Figure 3. $\mu_{\gamma \gamma}$ as function of $m_{H^{ \pm}}$for different values of $\lambda_{1}$ as defined in the inset. $1 \sigma$ and $2 \sigma$ limits of $\mu_{\gamma \gamma}$ from ATLAS are also shown in blue and orange colours for comparison purpose.

where $\mathcal{A}_{\mathrm{SM}}$ represents pure SM contribution (see [80-83]). And $F(x)=-[x-$ $f(x)] x^{-2}$ where

$$
f(x)= \begin{cases}\left(\sin ^{-1} x\right)^{2}, & x \leq 1 \\ -\frac{1}{4}\left[\ln \frac{1+\sqrt{1-x^{-1}}}{1-\sqrt{1-x^{-1}}}-i \pi\right]^{2} & x>1 .\end{cases}
$$

Therefore it turns out that IDM contribution to $\mu_{\gamma \gamma}$ is function of both mass of the charged Higgs $\left(m_{H^{ \pm}}\right)$and the coefficient of the trilinear coupling $h H^{+} H^{-}$i.e. $\lambda_{1}$. The measured value of $\mu_{\gamma \gamma}$ are given by $\mu_{\gamma \gamma}=1.17 \pm 0.27$ from ATLAS [84] and $\mu_{\gamma \gamma}=1.14_{-0.23}^{+0.26}$ from CMS [85]. In figure 3, we show the variation of $\mu_{\gamma \gamma}$ as function of $m_{H^{ \pm}}$for different values of $\lambda_{1}$. We also present the experimental limits on $\mu_{\gamma \gamma}$ from ATLAS in figure 3. Excepting for the resonance at $m_{h / 2}$, we see that our choice of $\lambda_{1}$ is consistent with experimental bound. The larger is $m_{H^{ \pm}} \mathrm{GeV}, \mu_{\gamma \gamma} \rightarrow 1$ i.e. approches to SM value. Also, we see that $\lambda_{1}>0$ diminishes $\mu_{\gamma \gamma}$, while $\lambda_{1}<0$ tends to enhance it. In this analysis, we mostly consider $m_{H^{ \pm}}>m_{h / 2}$ and positive $\lambda_{1}$ within correct experimental limit.

Relic density of DM. The PLANCK experiment [14] provides the observed amount of relic abundance

$$
0.1166 \leq \Omega_{\mathrm{DM}} h^{2} \leq 0.1206
$$

Furthermore, strong constraints exist from direct DM search experiments. In our analysis we will consider the most recent bound on direct detection cross section provided by XENON 1T [26]. Relic density and direct search allowed parameter space of the model will be evaluated in details.

Neutrino observables. The parameters associated to the neutrino sector should satisfy the bounds provided by different ongoing neutrino experiments. Limit on sum of light 

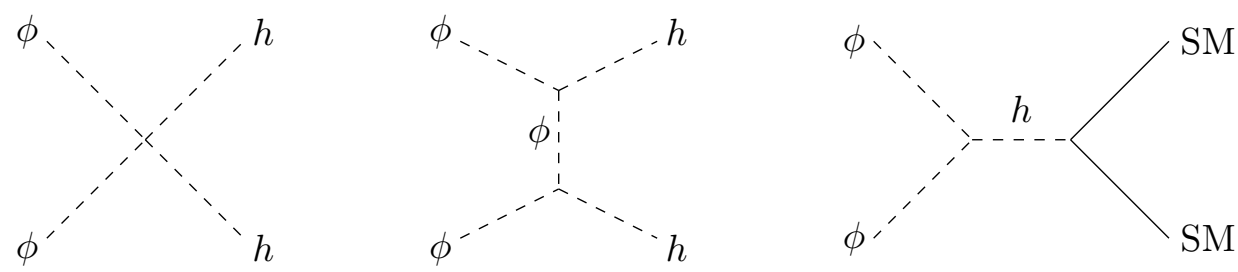

Figure 4. Annihilation processes of real scalar singlet DM $(\phi)$ to SM particles. SM in the last graph stands for $W^{ \pm}, Z, h$ and SM fermions.

neutrino masses $\sum m_{\nu_{i}} \leq 0.12 \mathrm{eV}$ as provided by PLANCK data $[14,86]$ is incorporated. The present values of neutrino mass hierarchies and mixing angle can be found in $[87,88]$. Due to the presence of $\mathrm{RH}$ neutrino in the set up, the constraint from lepton flavor violating decay (LFV) (dominantly from $\mu \rightarrow e \gamma$ ) will be applicable [89-91]. LFV constraint can be successfully evaded for $M_{N} \gtrsim 10^{3.5} \mathrm{GeV}[92,93]$ even for neutrino Yukawa coupling of $\mathcal{O}(1)$. It is important to note that in our model, the IDM does not interact with SM leptons and thus plays no role in LFV.

\section{Single component DM frameworks involving $\phi$ or $H^{0}$}

The model inherits two DM candidates: inert DM $H^{0}$ and singlet scalar DM $\phi$. Both the DM components have been studied extensively in literature as individual candidates to satisfy relic density and direct search bounds. Let us first revisit the single component frameworks for these two cases here. The relic density of scalar singlet $(\phi)$ is obtained via thermal freeze out through annihilation to SM through the Feynman graphs shown in figure 4. The direct search constraint for $\phi$ comes from the $t$ - channel Higgs portal interaction (turning the last graph of figure 4 upside down). The relevant parameters of the model are $[17,20,23]$ :

$$
\phi \text { as single component DM : }\left\{m_{\phi}, \lambda_{\phi h}\right\} .
$$

The allowed parameter space of $\phi$ is depicted in left hand side (l.h.s.) of figure 5 in $m_{\phi}-\lambda_{\phi h}$ plane by the red dots. Direct search allowed parameter space from XENON1T data $[26,27]$ using spin-independent DM-nucleon scattering cross section is shown by the blue dots. We therefore see that the model can only survive either in the Higgs resonance region $\left(\sim m_{h} / 2\right)$ or at a very heavy mass $\gtrsim 900 \mathrm{GeV}$. The under abundant (shown in yellow) and over abundant regions are also indicated.

IDM $\left(H^{0}\right)$ as a single component DM have annihilation and co-annihilation channels for freeze-out due to both gauge and Higgs portal interactions as shown by the Feynman graphs in figures 6 and 7. The parameters, which govern the IDM phenomenology are [94]:

$$
H^{0} \text { as single component DM: }\left\{m_{H^{0}}, m_{A^{0}}, m_{H^{ \pm}}, \lambda_{L}\right\} \text {. }
$$

Relic density allowed parameter space for single component IDM is shown in right hand side (r.h.s.) of figure 5 in $m_{H^{0}}-\lambda_{L}$ plane by red dots. Direct search (XENON1T data) 

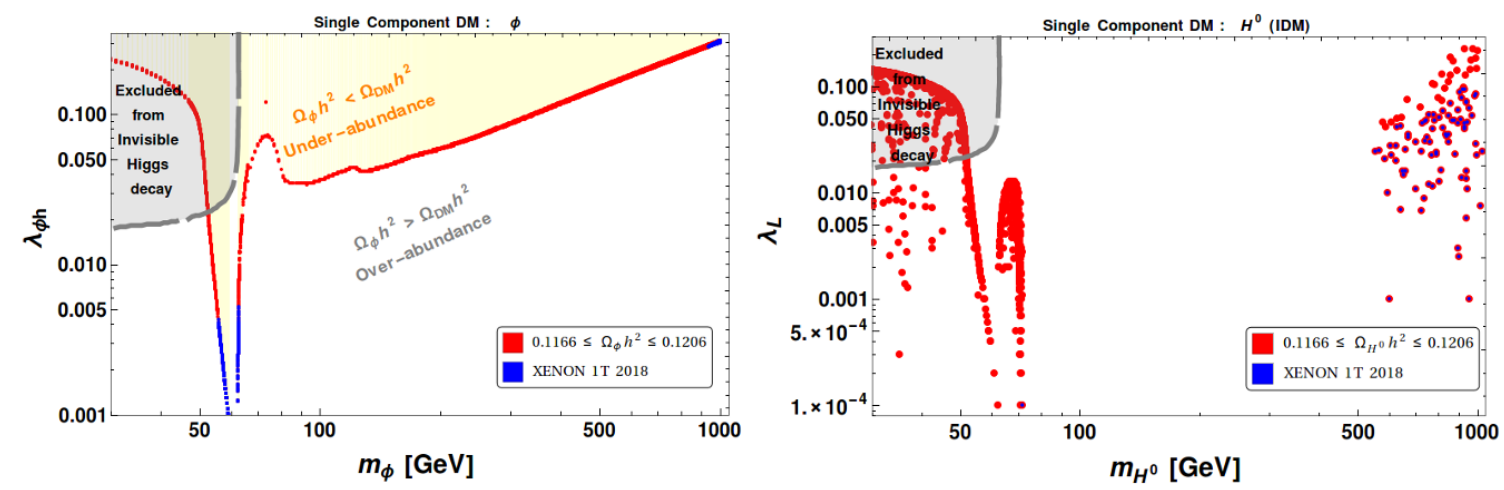

Figure 5. Relic density (red dots) and direct search/XENON1T (blue dots) allowed parameter space of the single component DM; scalar singlet $(\phi)$ on left (in $m_{\phi}-\lambda_{\phi h}$ plane) and IDM $\left(H^{0}\right)$ on right (in $m_{H^{0}}-\lambda_{L}$ plane). For the right hand side plot, we have used: $0 \leq m_{A^{0}}-m_{H^{0}} \leq 200 \mathrm{GeV}$, $1 \leq m_{H^{ \pm}}-m_{H^{0}} \leq 400 \mathrm{GeV}$, and $\lambda_{\Phi}=0.001$.
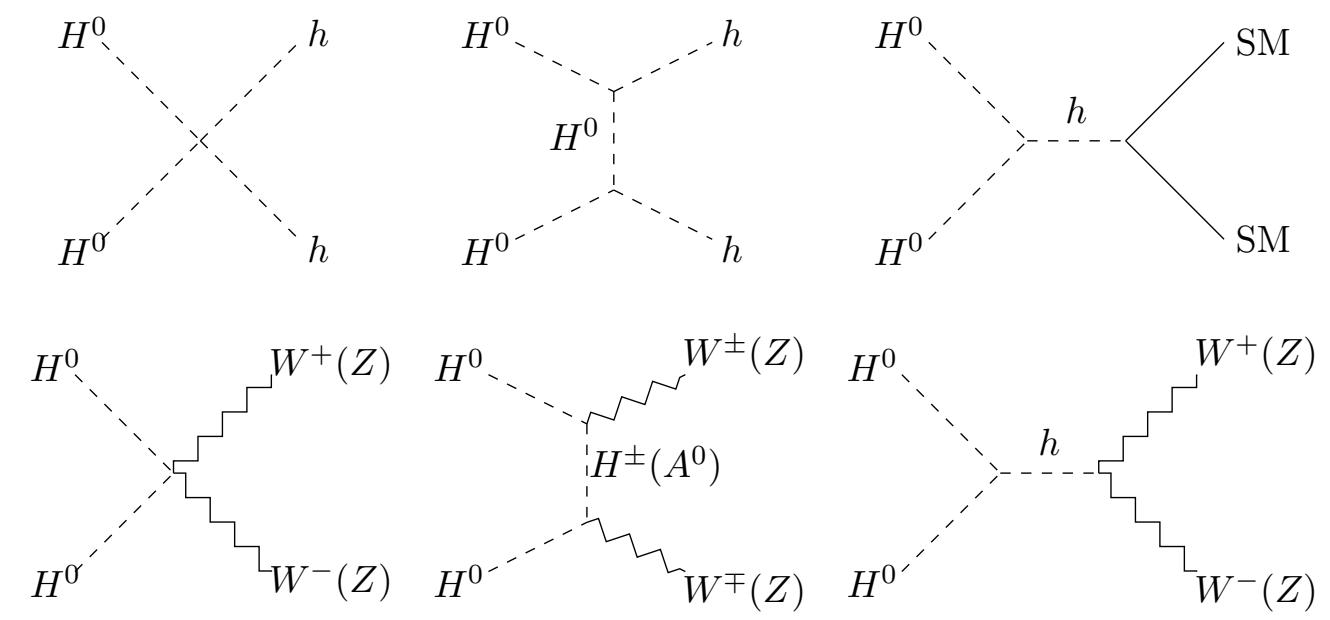

Figure 6. Annihilation processes of IDM $\left(H^{0}\right)$ to SM particles. SM in the top right graph stands for $W^{ \pm}, Z, h$ and SM fermions.

allowed points are shown by blue dots. The scan is obtained using $1 \leq m_{A^{0}}-m_{H^{0}} \leq$ $200 \mathrm{GeV}, 1 \leq m_{H^{ \pm}}-m_{H^{0}} \leq 400 \mathrm{GeV}$ with self coupling $\lambda_{\Phi}=0.001$ kept constant. Here we see again that allowed region from relic density and direct search constraint for IDM lies either in the small mass region $m_{H^{0}} \lesssim m_{W} \sim 80 \mathrm{GeV}$ or in the heavy mass region $m_{H^{0}} \gtrsim$ $550 \mathrm{GeV}$. It is a well known result coming essentially due to too much annihilation and coannihilation of IDM to SM through gauge interactions [94]. The disallowed region $80 \mathrm{GeV}<$ $m_{H^{0}}<550 \mathrm{GeV}$ is often called desert region, which is obviously under abundant. Another important point of single component IDM is that the direct search allowed parameter space beyond resonance $\left(m_{H^{0}} \gtrsim 550 \mathrm{GeV}\right)$ have significant co-annihilation dependence with $m_{H^{ \pm}}-m_{H^{0}} \lesssim 10 \mathrm{GeV}$ and $m_{A^{0}}-m_{H^{0}} \lesssim 10 \mathrm{GeV}$. 

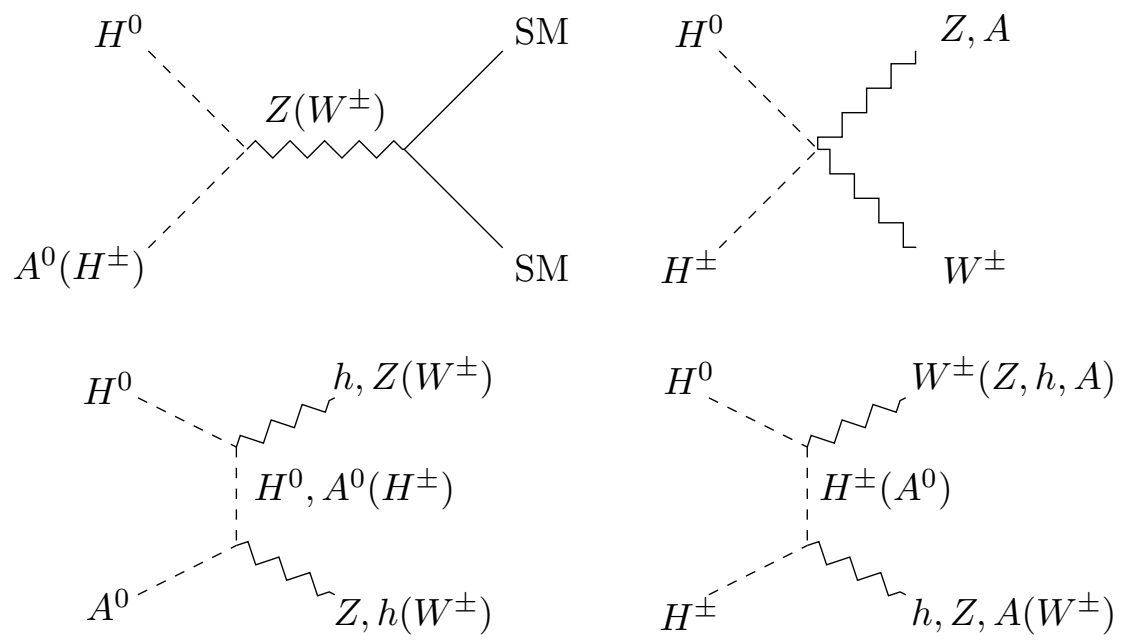

Figure 7. Co-annihilation processes of IDM $\left(H^{0}\right)$ with $A^{0}$ and $H^{ \pm}$to SM particles. SM in the top left graph stands for $W^{ \pm}, Z, h$ and SM fermions in suitable combination.
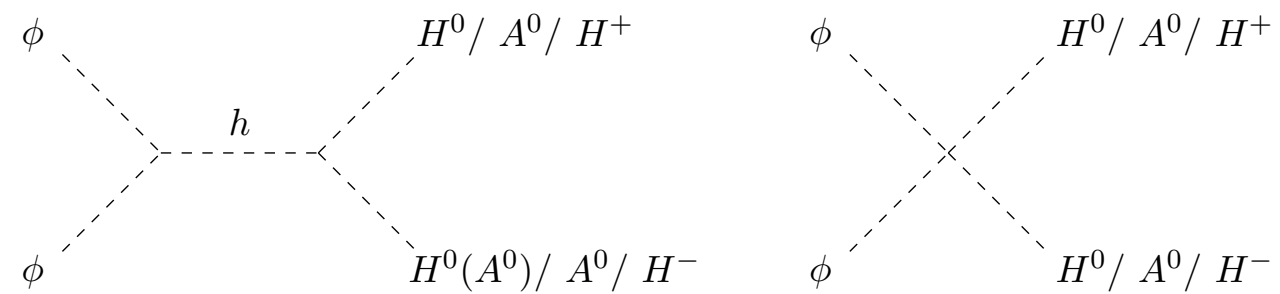

Figure 8. DM $\rightarrow$ DM conversion processes in a model with $\phi$ and $H^{0}$. We have assumed $m_{\phi}>$ $m_{H^{0}}, m_{H^{ \pm}}, m_{A^{0}}$ here. The reverse processes occur with reverse hierarchy.

\section{Two component DM set-up with $\phi$ and $H^{0}$}

\subsection{Coupled Boltzmann equations and direct search}

In presence of two DM components $\left(\phi\right.$ and $\left.H^{0}\right)$ DM-DM conversion plays a crucial role. The heavier DM can annihilate to the lighter component and thus contribute to the freezeout of heavier DM. The conversion processes are shown in figure 8, which shows that they are dictated by four point contact interactions as well as by Higgs portal coupling. It is clear that $H^{ \pm}, A^{0}$ are not really DM, but belongs to dark sector, hence annihilation to them is broadly classified within DM-DM conversion. More importantly none of them contribute to direct search. The two component DM set up therefore requires following parameters for analysis:

$$
\text { Two component DM : }\left\{m_{H^{0}}, m_{A^{0}}, m_{H^{ \pm}}, m_{\phi}, \lambda_{L}, \lambda_{\phi h}\right\} \text {. }
$$

There are two self interacting quartic couplings present in the model; namely $\lambda_{\Phi}$ and $\lambda_{\phi}$, which do not play an important role in DM analysis, but appear in vacuum stability constraint that we discuss later.

When $m_{\phi}>m_{H^{0}}, m_{H^{ \pm}}, m_{A^{0}}$, then $\phi$ can annihilate to all possible IDM components. The dominant $s$ - wave DM-DM conversion cross-sections $(\sigma v)$ of $\phi$ in non-relativistic ap- 
proximation are given by:

$$
\begin{aligned}
(\sigma v)_{\phi \phi \rightarrow H^{0} H^{0}} & =\frac{1}{64 \pi m_{\phi}^{2}}\left[\lambda_{c}+\frac{2 \lambda_{L} \lambda_{\phi h} v^{2}}{\left(4 m_{\phi}^{2}-m_{h}^{2}\right)}\right]^{2} \sqrt{1-\frac{4 m_{H^{0}}^{2}}{4 m_{\phi}^{2}}} \Theta\left(m_{\phi}-m_{H^{0}}\right) \\
(\sigma v)_{\phi \phi \rightarrow A^{0} A^{0}} & =\frac{1}{64 \pi m_{\phi}^{2}}\left[\lambda_{c}+\frac{2 \lambda_{S} \lambda_{\phi h} v^{2}}{\left(4 m_{\phi}^{2}-m_{h}^{2}\right)}\right]^{2} \sqrt{1-\frac{4 m_{A^{0}}^{2}}{4 m_{\phi}^{2}}} \Theta\left(m_{\phi}-m_{A^{0}}\right) \\
(\sigma v)_{\phi \phi \rightarrow H^{+} H^{-}} & =\frac{1}{32 \pi m_{\phi}^{2}}\left[\lambda_{c}+\frac{\lambda_{1} \lambda_{\phi h} v^{2}}{\left(4 m_{\phi}^{2}-m_{h}^{2}\right)}\right]^{2} \sqrt{1-\frac{4 m_{H^{ \pm}}^{2}}{4 m_{\phi}^{2}}} \Theta\left(m_{\phi}-m_{H^{ \pm}}\right),
\end{aligned}
$$

where $\lambda_{S}=\frac{1}{2}\left(\lambda_{1}+\lambda_{2}-\lambda_{3}\right)$. On the other hand, when $m_{\phi}<m_{H^{0}}$, the conversion process will be as $H^{0} H^{0}\left(A^{0}\right) \rightarrow \phi \phi$ or $H^{+} H^{-} \rightarrow \phi \phi$. The corresponding cross-sections can easily be gauged from eq. (5.2).

The evolution of DM number density for both components $\left(\phi\right.$ and $\left.H^{0}\right)$ in early universe as a function of time is obtained by coupled Boltzmann equations (CBEQ) as described in eq. (5.3):

$$
\begin{aligned}
& \frac{d n_{H^{0}}}{d t}+3 H n_{H^{0}}=-\sum_{X}\langle\sigma v\rangle_{H^{0}}{ }_{X \rightarrow \mathrm{SM} \mathrm{SM}}\left(n_{H^{0}} n_{X}-n_{H^{0}}^{e q} n_{X}^{e q}\right) \Theta\left(m_{H^{0}}+m_{X}-2 m_{\mathrm{SM}}\right), \\
& -\sum_{X}\langle\sigma v\rangle_{H^{0}}{ }_{X \rightarrow \phi \phi}\left(n_{H^{0}} n_{X}-\frac{n_{H^{0}}^{e q} n_{X}^{e q}}{n_{\phi}^{e q 2}} n_{\phi}^{2}\right) \Theta\left(m_{H^{0}}+m_{X}-2 m_{\phi}\right), \\
& +\sum_{X, Y}\langle\sigma v\rangle_{\phi \phi \rightarrow X Y}\left(n_{\phi}^{2}-\frac{n_{\phi}^{e q 2}}{n_{X}^{e q} n_{Y}^{e q}} n_{X} n_{Y}\right) \Theta\left(2 m_{\phi}-m_{X}+m_{Y}\right) ; \\
& \frac{d n_{\phi}}{d t}+3 H n_{\phi}=-\langle\sigma v\rangle_{\phi \phi \rightarrow \mathrm{SM} \mathrm{SM}}\left(n_{\phi}^{2}-n_{\phi}^{e q 2}\right) \Theta\left(m_{\phi}-m_{\mathrm{SM}}\right), \\
& -\sum_{X, Y}\langle\sigma v\rangle_{\phi \phi \rightarrow X Y}\left(n_{\phi}{ }^{2}-\frac{n_{\phi}^{e q 2}}{n_{X}^{e q} n_{Y}^{e q}} n_{X} n_{Y}\right) \Theta\left(2 m_{\phi}-m_{X}+m_{Y}\right), \\
& +\sum_{X}\langle\sigma v\rangle_{H^{0}}{ }_{X \rightarrow \phi \phi}\left(n_{H^{0}} n_{X}-\frac{n_{H^{0}}^{e q} n_{X}^{e q}}{n_{\phi}^{e q 2}} n_{\phi}^{2}\right) \Theta\left(m_{H^{0}}+m_{X}-2 m_{\phi}\right)
\end{aligned}
$$

where $\{X, Y\}=\left\{H^{0}, A^{0}, H^{ \pm}\right\}$. We can clearly spot DM-DM conversion contributions in second and third lines of each equation, which actually make the two equations 'coupled'. The freeze-out of two component DM is therefore obtained by numerically solving the above CBEQ and yields relic density (for a detailed discussion see for example [39]). The total relic density $\left(\Omega_{\mathrm{DM}}\right)$ will then have contributions from both DM components as:

$$
\Omega_{\mathrm{DM}} h^{2}=\Omega_{H^{0}} h^{2}+\Omega_{\phi} h^{2} .
$$

Now let us turn to direct search of two component DM set up. Both the DM candidates can be detected through the spin independent (SI) direct detection (DD) processes through $t$-channel Higgs mediation as depicted in figure 9. The SI DD cross section for $H^{0}\left(\sigma_{H^{0}}\right)$ and for $\phi\left(\sigma_{\phi}\right)$ at tree level turn out to be [20,39]:

$$
\sigma_{H^{0}}^{\text {eff, tree }}=\left(\frac{\Omega_{H^{0}} h^{2}}{\Omega_{\mathrm{DM}} h^{2}}\right) \frac{\lambda_{L}^{2} f_{N}^{2}}{\pi} \frac{\mu_{H^{0}, N}^{2} m_{N}^{2}}{m_{h}^{4} m_{H_{0}}^{2}}, \quad \sigma_{\phi}^{\text {eff, tree }}=\left(\frac{\Omega_{\phi} h^{2}}{\Omega_{\mathrm{DM}} h^{2}}\right) \frac{\lambda_{\phi h}^{2} f_{N}^{2}}{4 \pi} \frac{\mu_{\phi, N}^{2} m_{N}^{2}}{m_{h}^{4} m_{\phi}^{2}},
$$



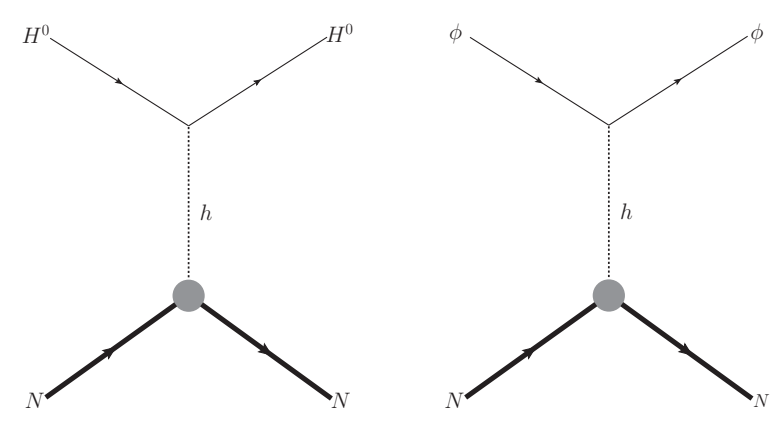

Figure 9. Spin independent direct detection processes for IDM (left) and scalar singlet DM (right).

where $\mu_{\phi, N}=\frac{m_{\phi} m_{N}}{m_{\phi}+m_{N}}$ and $\mu_{H^{0}, N}=\frac{m_{H^{0}} m_{N}}{m_{H^{0}}+m_{N}}$ are the reduced masses with nucleon, $f_{N}=$ 0.2837 represents the form factor of nucleon $[95,96]$ and $m_{N}=0.939 \mathrm{GeV}$ represents nucleon mass. Importantly, the effective direct search cross-section for each individual component is modified by the fraction with which it is present in the universe, ${ }^{2}$ given by $\frac{\Omega_{H^{0}} h^{2}}{\Omega_{\mathrm{DM}} h^{2}}$ for $H^{0}$ and $\frac{\Omega_{\phi} h^{2}}{\Omega_{\mathrm{DM}} h^{2}}$ for $\phi$. It is pertinent to note here that next to leading order (NLO) corrections to SI direct detection cross section can turn dominant for some region of parameter space in case of IDM [98, 99]. The NLO corrections arise mainly from one loop triangular and box diagrams mediated by gauge bosons and also from Higgs vertex corrections. In [99], authors extend the analysis of [98] to include gluon contribution at two loop and also contribution of IDM self coupling. In our case, the self coupling of IDM can be considered to be sufficiently small (as it is not playing any significant role in DM phenomenology) and hence we can ignore the effect of it. As shown in [99], in presence of loop effects, the coupling $\left(\lambda_{L}\right)$ that enters into $\sigma_{H^{0}}^{\text {eff }}$ gets replaced by $\lambda_{L}^{\text {eff }}=\lambda_{L}+\delta \lambda_{L}$ where $\delta \lambda_{L}$ takes into account the loop contributions effectively. Following this, we rewrite $\sigma_{H^{0}}^{\text {eff }}$ as

$$
\sigma_{H^{0}}^{\text {eff, } 1 \text { loop }}=\sigma_{H^{0}}^{\text {eff, tree }}(1+\kappa)
$$

where $\kappa$ is the enhancement factor to SI DD cross section of IDM candidate due to loop correction. It is shown in [98], $\kappa$ can take different values depending on $\lambda_{L}$. For smaller values of $\lambda_{L}, \kappa$ turns bigger; for example, with $0.001 \lesssim \lambda_{L}<0.01$ the correction turns out to be $100 \gtrsim \kappa>10$; for $0.01 \lesssim \lambda_{L}<0.1 \rightarrow 10 \gtrsim \kappa>1$ and $\kappa \lesssim 1$ for $\lambda_{L} \gtrsim 0.1$ for single component IDM relic density allowed points. Here we follow similar scaling and take $\kappa \sim \frac{0.1}{\lambda_{L}}$ to accommodate NLO corrections for IDM direct search in our analysis.

To obtain relic density and tree level DD cross sections of both the DM candidates numerically, we have used MicrOmegas [100]. It is noteworthy, that version 4.3 of MicrOmegas is capable of handling two component DM and we have cross-checked the solution from the code to match very closely to the numerical solution of CBEQ in eq. (5.3). For generating the model files compatible with MicrOmegas, we have implemented the model in LanHEP [101].

\footnotetext{
${ }^{2} \mathrm{~A}$ more comprehensive bound on multipartite DM from direct search can be obtained from the recoil rate of the nucleus [34, 97].
} 

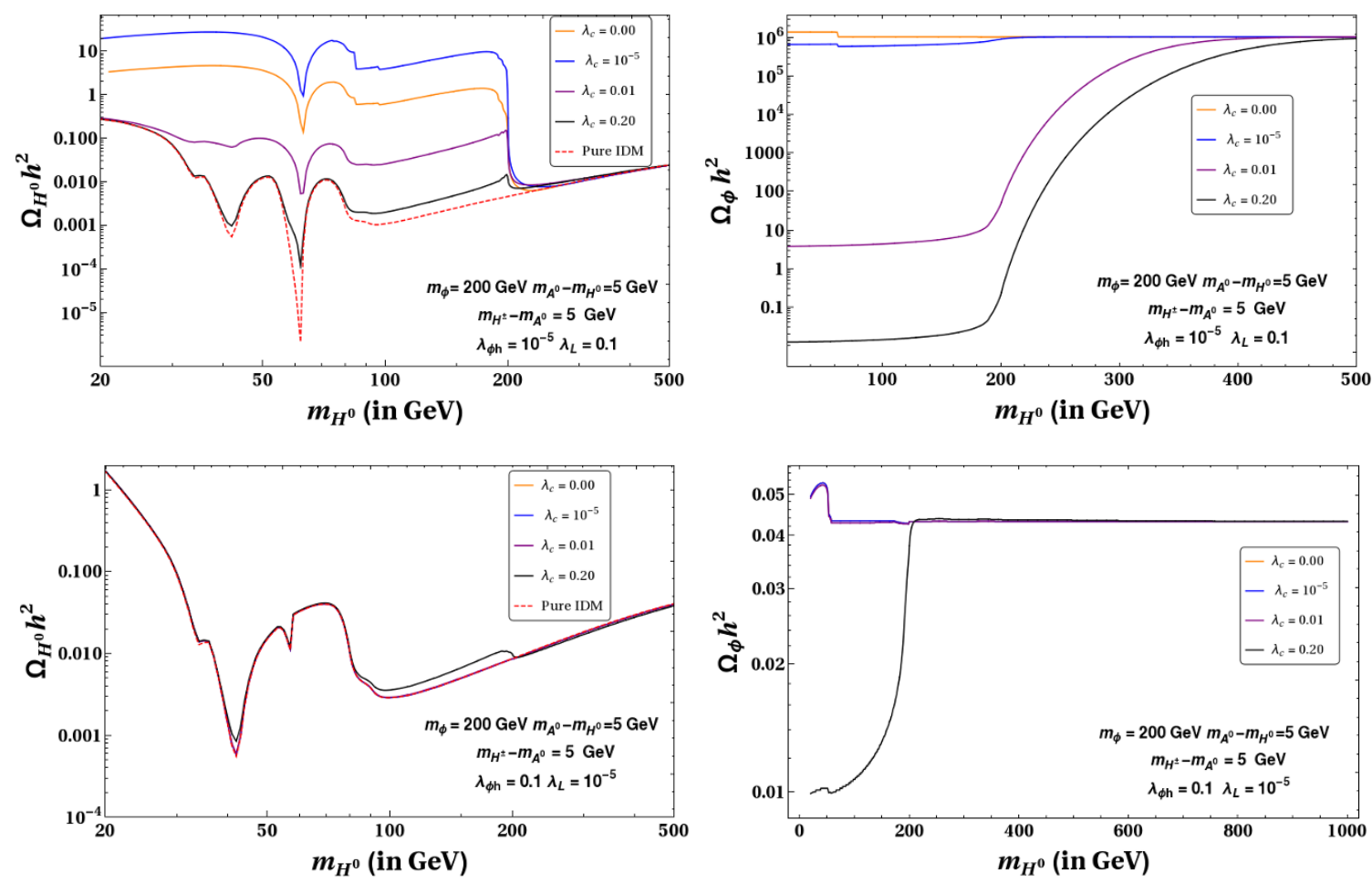

Figure 10. Relic Density of IDM, $H^{0}$ (left panel) and scalar DM, $\phi$ (right panel) as a function of IDM mass $m_{H^{0}}$, with different choices of $\lambda_{c}$. We illustrate two different combinations of DM-SM couplings, in the top panel: $\left\{\lambda_{\phi h}=10^{-5}, \lambda_{L}=0.1\right\}$ and in bottom panel: $\left\{\lambda_{\phi h}=0.1, \lambda_{L}=10^{-5}\right\}$. Other parameters kept fixed, are mentioned in the inset of each figure.

\subsection{Role of DM-DM conversion in relic density}

We first study the variation of relic density with respect to DM mass and other relevant parameters to extract the importance of DM-DM conversion in this two-component set up before elaborating on the relic density and direct search allowed parameter space of the model.

In figure 10 we plot relic densities of two DM candidates: $\Omega_{H^{0}} h^{2}$ in left panel and $\Omega_{\phi} h^{2}$ in right panel figures as function of $m_{H^{0}}$ for different values of $\lambda_{c}$. We also keep $m_{\phi}$ fixed at $200 \mathrm{GeV}$ here. Other parameters are chosen as mentioned in the inset of individual plots.

- Top left of figure 10: In this figure we have shown the variation of $\Omega_{H^{0}} h^{2}$ with $m_{H^{0}}$ for different values of $\lambda_{c}=0,10^{-5}, 0.01,0.2$ by yellow, blue, purple and black solid lines respectively. Pure IDM case (in a single component framework) is depicted by red dotted line, where also evidently $\lambda_{c}=0$. It is important to note the other parameters kept fixed for this plot are: $\lambda_{\phi h}=10^{-5}, \lambda_{L}=0.1, m_{H^{ \pm}}-m_{A^{0}}=m_{A^{0}}-m_{H^{0}}=5 \mathrm{GeV}$. We see that for $m_{H^{0}}<m_{\phi}$, relic density of $H^{0}$ changes significantly with the variation of $\lambda_{c}$. We also see that $\lambda_{c}=0$ (in two component set-up) is way above the pure IDM case yielding a large relic density. Now, with slight increase in $\lambda_{c}=10^{-5}$, relic density goes further up and then reduces significantly for larger $\lambda_{c}=0.01,0.2$. The interesting point is that the case of $\lambda_{c}=0.2$ lies very close to the pure IDM case. 
It is therefore evident that the presence of the second DM component $\phi$ plays an important role in $\Omega_{H^{0}} h^{2}$ through the coupling $\lambda_{c}$. For $m_{H^{0}}<m_{\phi}$, relic density of the heavier component $\phi$ can easily inherit the annihilation to other DM components $\{X, Y\}=\left\{H^{0}, A^{0}, H^{ \pm}\right\}$in addition to $\mathrm{SM}$ as $[39,102]$ :

$$
\Omega_{\phi} h^{2} \simeq \frac{854.45 \times 10^{-13} x_{f}}{\sqrt{g_{*}}\left(\langle\sigma v\rangle_{\phi \phi \rightarrow \mathrm{SM} \mathrm{SM}}+\langle\sigma v\rangle_{\phi \phi \rightarrow X Y}\right)} \simeq \frac{0.1 \mathrm{pb}}{\langle\sigma v\rangle_{\phi \phi \rightarrow \mathrm{SM} \mathrm{SM}}+\langle\sigma v\rangle_{\phi \phi \rightarrow X Y}},
$$

where in the last step, we have used $g_{*}=106.7$ and $x_{f}=20$ [102]. However it is difficult to envisage the relic density for the lighter DM component due to such DM-DM conversion. This can be understood from the CBEQ for the two component $\mathrm{DM}$ as in eq. (5.3). Let us define the following notations first:

$$
\begin{aligned}
\langle\sigma v\rangle_{H^{0} X \rightarrow \mathrm{SM}} n_{H^{0}} n_{X} & =\mathcal{F}_{H^{0}} ; & \langle\sigma v\rangle_{\phi \phi \rightarrow X Y} n_{\phi}^{2} & =\mathcal{F}_{\phi \Phi} ; \\
\langle\sigma v\rangle_{\phi \phi \rightarrow \mathrm{SM}} n_{\phi}^{2} & =\mathcal{F}_{\phi} ; & \langle\sigma v\rangle_{H^{0} X \rightarrow \phi \phi} n_{H^{0}} n_{X} & =\mathcal{F}_{\Phi \phi} .
\end{aligned}
$$

Again $\{X, Y\}=H^{0}, A^{0}, H^{ \pm}$; as earlier. The CBEQ with above notation, turns out to be (assuming $m_{\phi}>m_{H^{0}}$ ):

$$
\begin{aligned}
\frac{d n_{H^{0}}}{d t}+3 H n_{H^{0}} & \simeq-\mathcal{F}_{H^{0}}+\mathcal{F}_{\phi \Phi} ; \\
\frac{d n_{\phi}}{d t}+3 H n_{\phi} & \simeq-\mathcal{F}_{\phi}-\mathcal{F}_{\phi \Phi} .
\end{aligned}
$$

In eq. (5.9), we neglected the equilibrium number densities as they are tiny near freeze-out, where the dynamics is under study. With $\lambda_{c}=0$, and $\lambda_{\phi h}=10^{-5}$, annihilation cross-sections for $\phi,\left(\langle\sigma v\rangle_{\phi \phi \rightarrow \mathrm{SM} \text { SM }}\right.$ and $\left.\langle\sigma v\rangle_{\phi \phi \rightarrow \mathrm{X} \mathrm{Y}}\right)$ are very small. Hence $\phi$ freezes out early and the number density of $\phi$ turns out to be large since $n_{\phi} \propto 1 /\langle\sigma v\rangle_{\phi}^{\mathrm{eff}}$ where $\langle\sigma v\rangle_{\phi}^{\mathrm{eff}} \simeq\left(\langle\sigma v\rangle_{\phi \phi \rightarrow \mathrm{SM} \mathrm{SM}}+\langle\sigma v\rangle_{\phi \phi \rightarrow X Y}\right)$ following eq. (5.7). Now, it is easy to appreciate that with $\lambda_{c}=0$, and $\lambda_{\phi h}=10^{-5}$, annihilation of $\phi$ to $\mathrm{SM}$ is larger than conversion to other DM $\left(H^{0}\right)$, i.e. $\langle\sigma v\rangle_{\phi \phi \rightarrow \mathrm{SM} \mathrm{SM}} \gg\langle\sigma v\rangle_{\phi \phi \rightarrow X Y}$. However due to large $\phi$ abundance ${ }^{3}\left(n_{\phi} \sim 0.1 \mathrm{~cm}^{-3}\right), \mathcal{F}_{\phi \Phi}$ becomes comparable with $\mathcal{F}_{H^{0}}$. As these two terms $\left(\mathcal{F}_{\phi \Phi}\right.$ and $\left.\mathcal{F}_{H^{0}}\right)$ appear in the evolution of $n_{H^{0}}$ (eq. (5.9)) with opposite sign, it is quite evident that effective annihilation cross-section for $H^{0}$ becomes small and hence $n_{H^{0}}$ after freeze out turns out to be much larger than the pure IDM case. Next let us consider non zero but small $\lambda_{c}\left(=10^{-5}\right)$. Then $\langle\sigma v\rangle_{\phi \phi \rightarrow X Y}$ increases compared to the earlier case of $\lambda_{c}=0$. However due to smallness of the coupling $\lambda_{c}$, this does not make any significant change in the number density of $\phi$ and $n_{\phi} \sim 0.1 \mathrm{~cm}^{-3}$ remains the same (as $\phi \phi \rightarrow$ SM SM still dominantly contributes to the total annihilation cross section of $\phi$ ). Therefore, $\mathcal{F}_{\phi \Phi}$ increases and reduces the separation with $\mathcal{F}_{H^{0}}$. Hence the effective annihilation cross-section for $H^{0}$ turns out to be even smaller than $\lambda_{c}=0$ case. Therefore, for $\lambda_{c}=10^{-5}$ relic density increases further than that of $\lambda_{c}=0$. For larger value of $\lambda_{c}=0.01$, contribution from DM-DM conversion, $\langle\sigma v\rangle_{\phi \phi \rightarrow X Y}$ significantly rises and therefore

\footnotetext{
${ }^{3} n_{\phi}$ can be calculated by using the formula $\Omega_{\phi}=\frac{n_{\phi} m_{\phi}}{\rho_{c}}$, where $\rho_{c}=1.05 \times 10^{-5} h^{2} \mathrm{GeV} \mathrm{cm}^{-3}[102]$.
} 
the number density of $n_{\phi}$ drops to $n_{\phi} \sim 10^{-5} \mathrm{~cm}^{-3}$, and therefore $\mathcal{F}_{\phi \Phi}$ becomes much smaller than $\mathcal{F}_{H^{0}}$. This increases the effective annihilation for $H^{0}$ and reduces relic density. This trend continues for higher values of $\lambda_{c}$ and eventually leads to a vanishingly small $\mathcal{F}_{\phi \Phi}$ to closely mimic the case of single component IDM. The case of $m_{H^{0}}>m_{\phi}$ can also be understood from the CBEQ in this limit:

$$
\begin{aligned}
\frac{d n_{H^{0}}}{d t}+3 H n_{H^{0}} & \simeq-\mathcal{F}_{H^{0}}-\mathcal{F}_{\Phi \phi} ; \\
\frac{d n_{\phi}}{d t}+3 H n_{\phi} & \simeq-\mathcal{F}_{\phi}+\mathcal{F}_{\Phi \phi} .
\end{aligned}
$$

For $m_{H^{0}}>m_{\phi}$, relic density of $H^{0}$ can be written simply as

$$
\Omega_{H^{0}} h^{2} \simeq \frac{0.1 \mathrm{pb}}{\langle\sigma v\rangle_{H^{0} X \rightarrow \mathrm{SM} \mathrm{SM}}+\langle\sigma v\rangle_{H^{0} X \rightarrow \phi \phi}} .
$$

The annihilation to SM $\left(\langle\sigma v\rangle_{H^{0} X \rightarrow \mathrm{SM} \mathrm{SM}}\right)$ due to gauge coupling is much larger than the conversion cross-section $\langle\sigma v\rangle_{H^{0} X \rightarrow \phi \phi}$ for all the choices of $\lambda_{c}$, so we do not find any distinction between all those cases.

- Top right of figure 10: In the top right panel, the same parameter space is used to show the variation of $\Omega_{\phi} h^{2}$ with respect to $m_{H^{0}}$. The dynamics is much simpler for $m_{\phi}>m_{H^{0}}$ (see BEQ. (5.9)) where $\Omega_{\phi} h^{2} \simeq \frac{0.1 \mathrm{pb}}{\langle\sigma v\rangle_{\phi \phi \rightarrow \mathrm{SM} \mathrm{SM}}+\langle\sigma v\rangle_{\phi \phi \rightarrow X Y}}$. With larger $\lambda_{c}$, the conversion to other $\mathrm{DM}\left(\mathcal{F}_{\phi \Phi}\right)$ becomes larger and relic density drops accordingly. For $m_{\phi}<m_{H^{0}}$, we see from eq. (5.10), that there is a competition between $\mathcal{F}_{\phi}$ and $\mathcal{F}_{\Phi \phi}$. With increasing $m_{H^{0}},\langle\sigma v\rangle_{H^{0} X \rightarrow \phi \phi}$ decreases, therefore $\mathcal{F}_{\Phi \phi}$ decreases and eventually it becomes vanishingly small for $m_{H^{0}} \gtrsim 400 \mathrm{GeV}$. The equation for $n_{\phi}$ then becomes equivalent to the single component case of $\phi$ where $m_{H^{0}}$ is no more relevant for $\Omega_{\phi} h^{2}$.

- The bottom panel figures of figure 10 essentially indicate that with larger $\lambda_{\phi h}$, annihilation of $\phi$ to SM becomes large, resulting a smaller $n_{\phi}$ after freeze out. Therefore $\mathcal{F}_{\phi \Phi}$ in eq. (5.9) turns insignificant. On the other hand, $\mathcal{F}_{\Phi \phi}$ also becomes smaller than $\mathcal{F}_{\phi}$ in eq. (5.10). Together, relic density of the lighter DM component is not affected by the presence of a heavy DM component.

Before we move on, let us summarise the outcome of figure 10. We see here that relic density of lighter DM component is affected by the heavier one, when the annihilation cross-section to SM is tiny.

In figure 11. we plot the variation of IDM relic density as function of DM mass $m_{H^{0}}$ for different choices of $\Delta m=m_{A^{0}}-m_{H^{0}}:\{1-50\} \mathrm{GeV}$ shown by different coloured lines. The other parameters are kept fixed and mentioned explicitly in the figure insets. Let us first focus on the left panel plot. We notice that for $m_{H^{0}}<m_{W}$, with larger $\Delta m=$ $m_{A^{0}}-m_{H^{0}}$, relic density becomes larger. Keeping in mind that in this case $\left(m_{H^{0}}<m_{W}\right)$, co-annihilations (see figure 7) dominate the total $\langle\sigma v\rangle$, this can be explained from the usual convention of co-annihilation cross-section being reduced by Boltzmann suppression $(\sim$ 

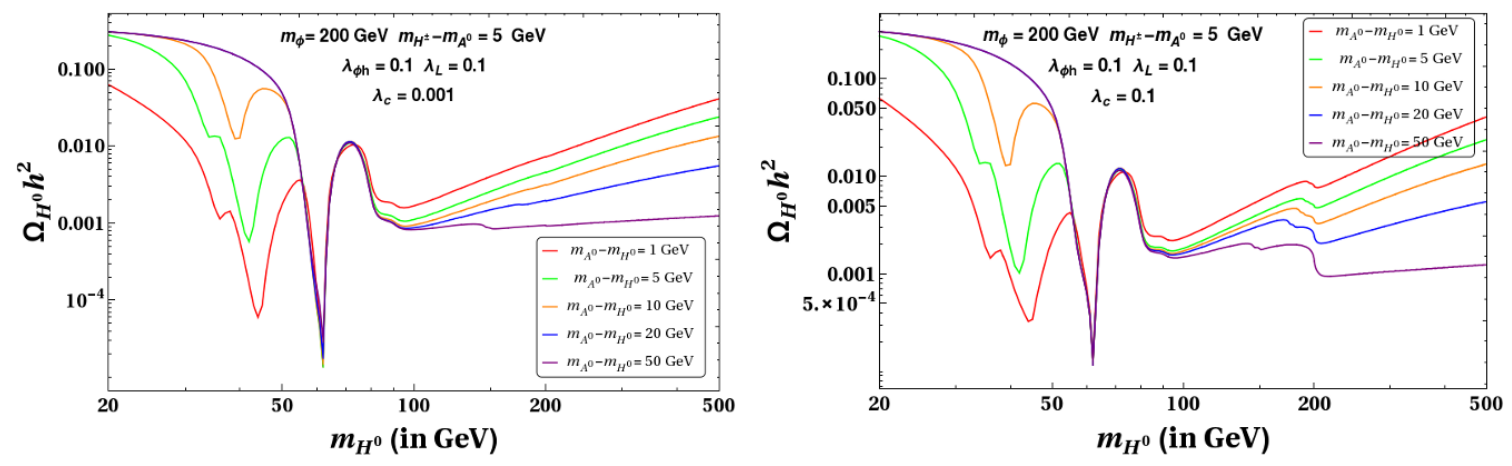

Figure 11. $\Omega_{H^{0}} h^{2}$ vs $\mathrm{m}_{\mathrm{H}^{0}}$ for $\lambda_{c}=0.001$ (left panel) and $\lambda_{c}=0.1$ (right panel) with different choices of $m_{A^{0}}-m_{H^{0}}$ which are depicted by different coloured lines in the figure. The other parameters kept fixed are mentioned in the inset of each figure.

$\left.e^{-\Delta m / T}\right)$ with larger splitting $(\Delta m)$. However, for $m_{H^{0}}>m_{W}$, the phenomena seems to be reversed, i.e., with larger mass splitting, relic density decreases. Note that for $m_{H^{0}}>m_{W}$, DM annihilations (in particular, annihilations into gauge bosons) become dominant. It turns out that the contribution of $\langle\sigma v\rangle_{H^{0} H^{0} \rightarrow W^{+} W^{-} \text {or } Z Z}$ increases with larger $\Delta m$ (for the relevant cross-section, see [41]). This happens due to presence of the destructive interference between the $s$ and $t$ channel contributions. With larger $\Delta m$, the destructive interference reduces and hence the annihilation cross section increases which in turn lowers the relic density of $H^{0}$ in this region. The same feature prevails in the right panel figure. Here, for a larger $\lambda_{c}$, the relic density of $H^{0}$ goes further down due to annihilation to $\phi$ beyond $m_{H^{0}}>m_{\phi}$.

\section{Relic density and direct search allowed parameter space}

One of the important motivations of this analysis is to study interacting multi-component DM phenomenology for DM mass lying between $80 \leq m_{\mathrm{DM}} \leq 500 \mathrm{GeV}$ for both the components in view of relic density $\left(0.1166 \leq \Omega_{\mathrm{DM}} h^{2}\left(\equiv \Omega_{H^{0}} h^{2}+\Omega_{\phi} h^{2}\right) \leq 0.1206\right.$ [14] $)$ and direct detection (XENON 1T [26]) bounds. We would like to recall that for the individual scenarios, none of the DM components satisfy relic and direct detection bounds simultaneously within this mass range (see section 4). Now in order to find a consistent parameter space in the set up, we perform a numerical scan of the relevant parameters within the specified ranges as mentioned below.

$$
\begin{aligned}
& 80 \leq m_{H^{0}} \leq 500 \mathrm{GeV}, 80 \leq m_{\phi} \leq 500 \mathrm{GeV}, \\
& 0 \leq m_{A^{0}}-m_{H^{0}} \leq 200 \mathrm{GeV}, 0 \leq m_{H^{ \pm}}-m_{A^{0}} \leq 180 \mathrm{GeV}, \\
& 0.001 \leq \lambda_{L} \leq 0.30, \quad 0.001 \leq \lambda_{\phi h} \leq 0.20, \quad 0.01 \leq \lambda_{c} \leq 1.00
\end{aligned}
$$

We also note here that as $\lambda_{L}$ and $\lambda_{\phi h}$ enters into direct search cross-sections for $H^{0}$ and $\phi$ DMs respectively, we keep those couplings in a moderate range, while $\lambda_{c}$ governs DM-DM interactions, but do not directly enter into direct search bounds, therefore we choose a 

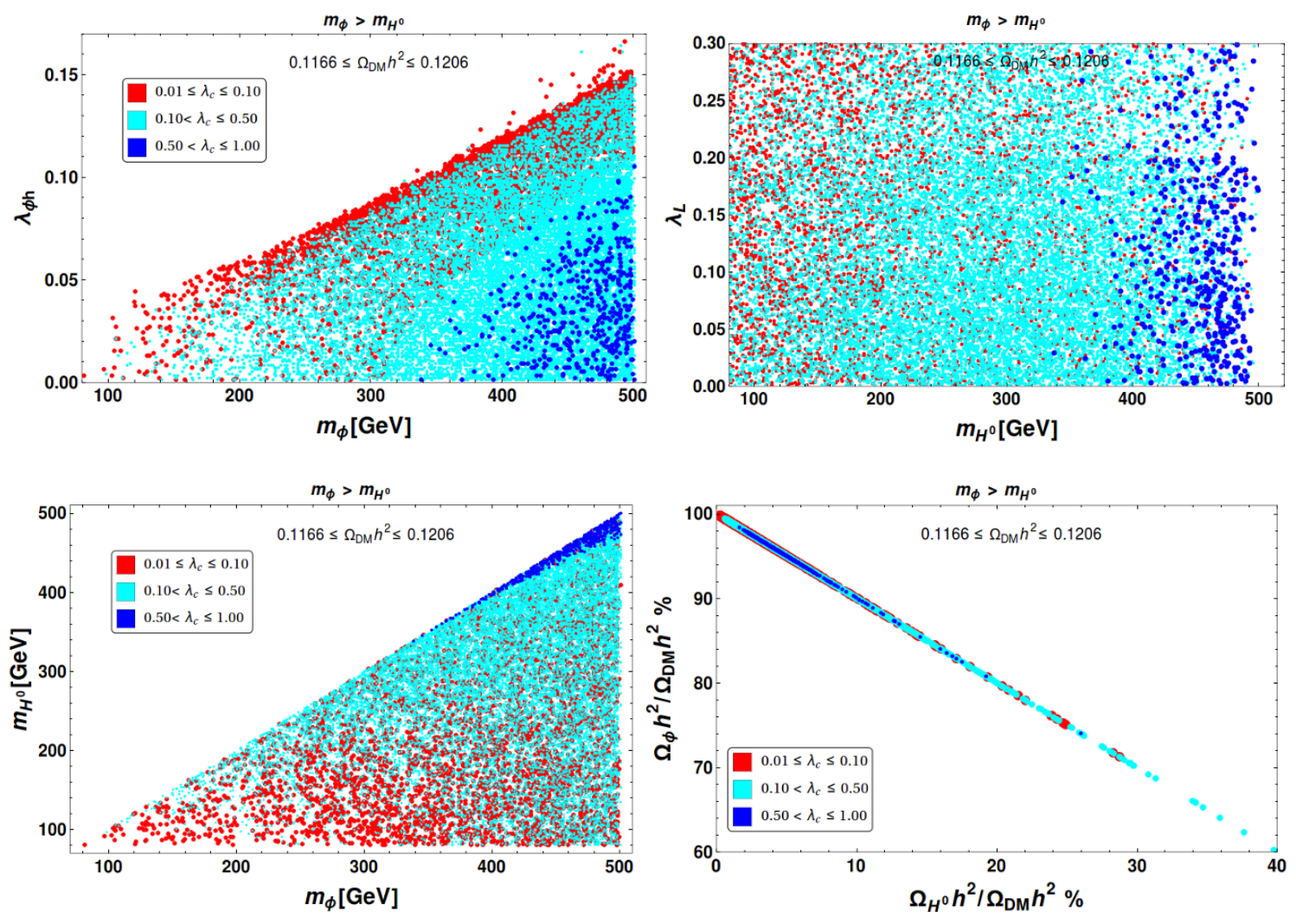

Figure 12. Relic Density allowed parameter space is shown in $m_{\phi}-\lambda_{\phi h}$ plane (top left), $m_{H^{0}}-\lambda_{L}$ plane (top right), $m_{\phi}-m_{H^{0}}$ plane (bottom left) and $\Omega_{\phi} h^{2} / \Omega_{\mathrm{DM}} h^{2}(\%)-\Omega_{H^{0}} h^{2} / \Omega_{\mathrm{DM}} h^{2}(\%)$ plane (bottom right) for the mass hierarchy $m_{\phi}>m_{H^{0}}$.

larger range (with natural values) for scanning $\lambda_{c} \cdot \lambda_{\phi}=0.001$ and $\lambda_{\Phi}=0.001$ are kept fixed throughout the analysis since those are not relevant for DM phenomenology.

There are two possible mass hierarchies for the two-component DM set up relevant for phenomenological analysis: (i) $m_{\phi}>m_{H^{0}}$ and (ii) $m_{\phi} \leq m_{H^{0}}$, which we address separately below.

\section{Case I: $m_{\phi}>m_{H^{0}}$}

Primarily, in such a scenario, the main physics arises due to annihilation of $\phi$ to $H^{0}$, on top of their individual annihilation to SM to govern the freeze-out.

In figure 12, we show relic density allowed parameter space for the model in terms of different relevant parameters. In top left panel of figure 12, we have shown the relic density satisfied (considering contribution from both $\phi$ and $H^{0}$ components) points in $m_{\phi}-\lambda_{\phi h}$ plane for different ranges of $\lambda_{c}$, as depicted in the figure with different colour codes. As seen from the plot, for a fixed $m_{\phi}$, there is a maximum $\lambda_{\phi h}$. All possible values less than the maximum $\lambda_{\phi h}$ is also allowed subject to different choices of $\lambda_{c}$. The larger is $\lambda_{c}$, the smaller is the required $\lambda_{\phi h}$ thanks to the conversion of $\phi \rightarrow H^{0}$ to yield relic density. It is also noted that for large $\lambda_{c} \gtrsim 0.5$, as the DM-DM conversion is very high, the DM mass $m_{\phi}$ has to lie in the high mass region $(\gtrsim 400 \mathrm{GeV})$ to tame the annihilation cross-section within 
acceptable range. To summarise, this figure shows that due to the presence of second DM component, much larger parameter space (actually the over-abundant regions of the single component framework, compare figure 5) is allowed. Top right panel figure shows the relic density allowed parameter space in $m_{H^{0}}-\lambda_{L}$ plane again for different ranges of $\lambda_{c}$ as in the left plot. It naturally depicts that $\lambda_{L}$ is insensitive to $m_{H^{0}}$ as the annihilation and co-annihilation cross-section of $H^{0}$ is mainly dictated by gauge interaction. However, we see a mild dependence on $\lambda_{c}$, such that when $\lambda_{c} \gtrsim 0.5$, the DM mass $m_{H^{0}}$ has to be heavy $\gtrsim 400 \mathrm{GeV}$. This is because with large $\lambda_{c}$, DM-DM conversion is large; to achieve relic density of correct order, $m_{\phi}$ requires to be large and the conversion can only be tamed down by phase space suppression, i.e. by choosing $H^{0}$ mass as close as possible to $\phi$ mass $\left(m_{H^{0}} \sim m_{\phi}\right)$. Bottom left figure correlates the DM masses to obtain correct density within $m_{\phi}>m_{H^{0}}$. We see that for small $\lambda_{c}$, particularly with higher $m_{\phi}$, large $m_{H^{0}}$ values are disfavoured in order to keep the DM-DM conversion in the right order. While for large $\lambda_{c}$, mass degeneracy is required $\left(m_{H^{0}} \sim m_{\phi}\right)$ to tame the DM-DM conversion. Bottom right figure shows the relative contribution of relic density of the two DM components. First of all, this shows that $\phi$ contributes with larger share of relic density, while the relic density of $H^{0}$ can at most be $40 \%$ of the total. This is natural from the perspective of IDM to be the lighter DM component, as we know it is always suppressed below $500 \mathrm{GeV}$ (with a maximum contribution 40\%) and the conversion channels do not alter IDM's annihilation for $m_{\phi}>m_{H^{0}}$. Although for $0.01<\lambda_{c} \lesssim 0.1$, individual relic abundance of IDM could be large for very small (and fine tuned) $\lambda_{\phi h} \sim 10^{-5}$, as shown in upper left of figure 10; with the increase of $\lambda_{\phi h}$, this enhancement vanishes. Since we scan the parameter space where $\lambda_{\phi h} \gtrsim 0.001$, such effect can not be found in figure 12. For small $\lambda_{c}$, contribution from $H_{0}$ is smaller, as relic density contribution from $\phi$ gets larger due to small DM-DM conversion. With larger $\lambda_{c} \gtrsim 0.1$, the DM-DM conversion for $\phi$ becomes larger and therefore the relic density of $\phi$ can easily span between $60-100 \%$. With very high $\lambda_{c} \sim 1$, DM-DM conversion becomes too large, therefore to keep relic density in the correct ballpark, the DM mass $\left(m_{\phi}\right)$ has to be heavy and almost degenerate with the heavier DM $\left(m_{H^{0}} \sim m_{\phi}\right)$. $\lambda_{\phi h}$ in such cases, requires to be very small, which are validated by some dark blue points with $\Omega_{\phi} h^{2} / \Omega_{\mathrm{DM}} h^{2} \sim 60 \%$.

Next in figure 13, we show the SI direct detection cross section for $H^{0}$ at relic density satisfied points in $\sigma_{H^{0}}^{\text {eff }}-m_{H^{0}}$ plane. Direct search cross-section at tree level is shown by light black points and NLO effects as prescribed in eq. (5.6), is shown by orange points. For comparison, we also point out the most recent XENON 1T experimental bound in dotted red line. Upper left panel of figure 13 shows that for $0.001 \lesssim \lambda_{L}<0.01$, the one loop correction to $\sigma_{H^{0}}^{\text {eff }}$ is really significant considering $\kappa \sim \frac{0.1}{\lambda_{L}}$ and rules out some of the DD bound satisfied points at tree level. Similar kind of feature can be found for $0.01 \lesssim \lambda_{L}<0.1$ case (upper right panel figure) also. Once $\lambda_{L} \gtrsim 0.1$, as in the lower panel of figure $13, \sigma_{H^{0}}^{\text {eff }}$ at NLO shows minor difference to tree level computation.

Relic density allowed parameters space consistent with direct search constraints at NLO where both DMs $\phi$ and $H^{0}$ simultaneously satisfy XENON $1 \mathrm{~T} 2018$ [103] bound (for different ranges of $\lambda_{c}$ ) are shown next in figure 14. This is illustrated in $m_{\phi}-\lambda_{\phi h}$ plane (top left panel), $m_{H^{0}}-\lambda_{L}$ plane (top right panel) and in $m_{\phi}-m_{H^{0}}$ plane (bottom panel) 

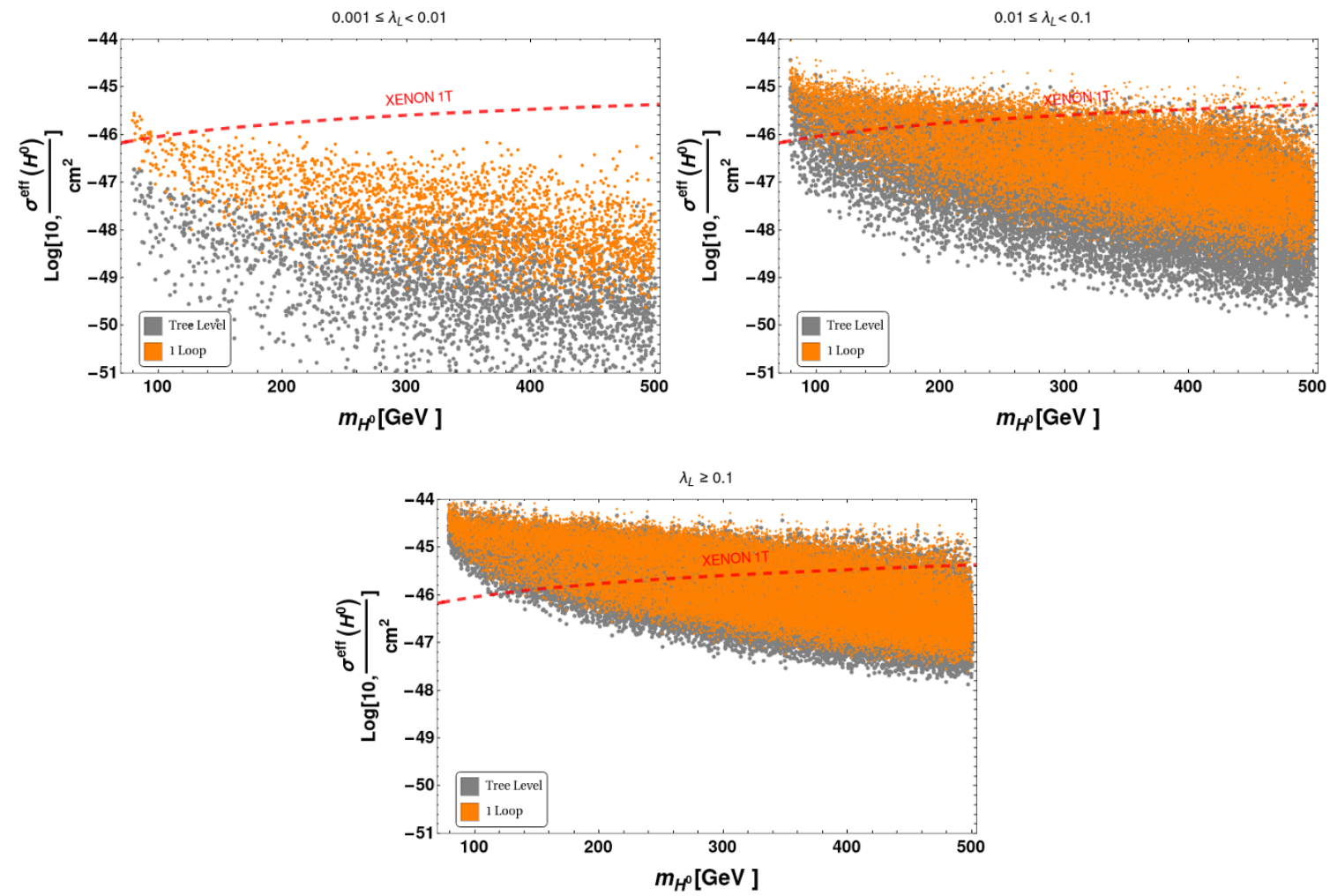

Figure 13. SI Direct detection cross section for IDM component is plotted against $m_{H^{0}}$ for (upper left) $0.001 \lesssim \lambda_{L}<0.01$, (upper right) $0.01 \lesssim \lambda_{L}<0.1$ and (bottom) $\lambda_{L} \gtrsim 0.1$. All the figures show $\sigma_{H^{0}}^{\text {eff }}$ both at tree level (light black) and at NLO level (orange). The XENON 2018 bound (red dotted line) is shown for comparison purpose.

similar to figure 12. We have already mentioned that spin independent (SI) DM-nucleon cross-section depends on square of Higgs portal couplings of the respective DM candidates, $\lambda_{\phi h}$ for $\phi$ and $\lambda_{L}$ for $H^{0}$ scaled by a pre-factor of the relative number density $\Omega_{i} h^{2} / \Omega_{\mathrm{DM}} h^{2}$ $\left(i=\phi, H^{0}\right)$. Since in this two component scenario, the dominant contribution is coming from $\phi \mathrm{DM}$, the pre-factor $\Omega_{\phi} h^{2} / \Omega_{\mathrm{DM}} h^{2} \sim 1$. On the other hand, for $H^{0}$ the pre-factor is small, $\Omega_{H^{0}} h^{2} / \Omega_{\mathrm{DM}} h^{2}<1$, and will help $H^{0}$ reducing the effective direct search crosssection. Therefore, portal coupling $\lambda_{\phi h}$ is tightly constrained from XENON $1 \mathrm{~T}$ bound to $\lambda_{\phi h} \lesssim 0.1$ for DM mass $m_{\phi} \lesssim 500 \mathrm{GeV}$, as shown in top left panel of figure 14 (compare it with the top left panel of figure 12). Similarly in top right panel, the direct search allowed $m_{H^{0}}-\lambda_{L}$ plane for $H^{0}$ shows that a large region corresponding to higher $\lambda_{L}$ is excluded as a function of $m_{H^{0}}$ (again, compare it with top right panel of figure 12). A possible mass correlation after direct search bound are plotted in bottom panel of figure 14 in $m_{\phi}-m_{H^{0}}$ plane. The main outcomes from this figure are: (i) For $\lambda_{c} \leq 0.1$, small $m_{H^{0}} \sim 200 \mathrm{GeV}$ is favoured, (ii) for moderate values of $\lambda_{c} \sim\{0.1-0.5\}$, there is no correlation and (iii) for large $\lambda_{c}$, only degenerate mass scenario $\left(m_{\phi} \sim m_{H^{0}}\right)$ with large $m_{\phi} \sim 400 \mathrm{GeV}$ is allowed. 

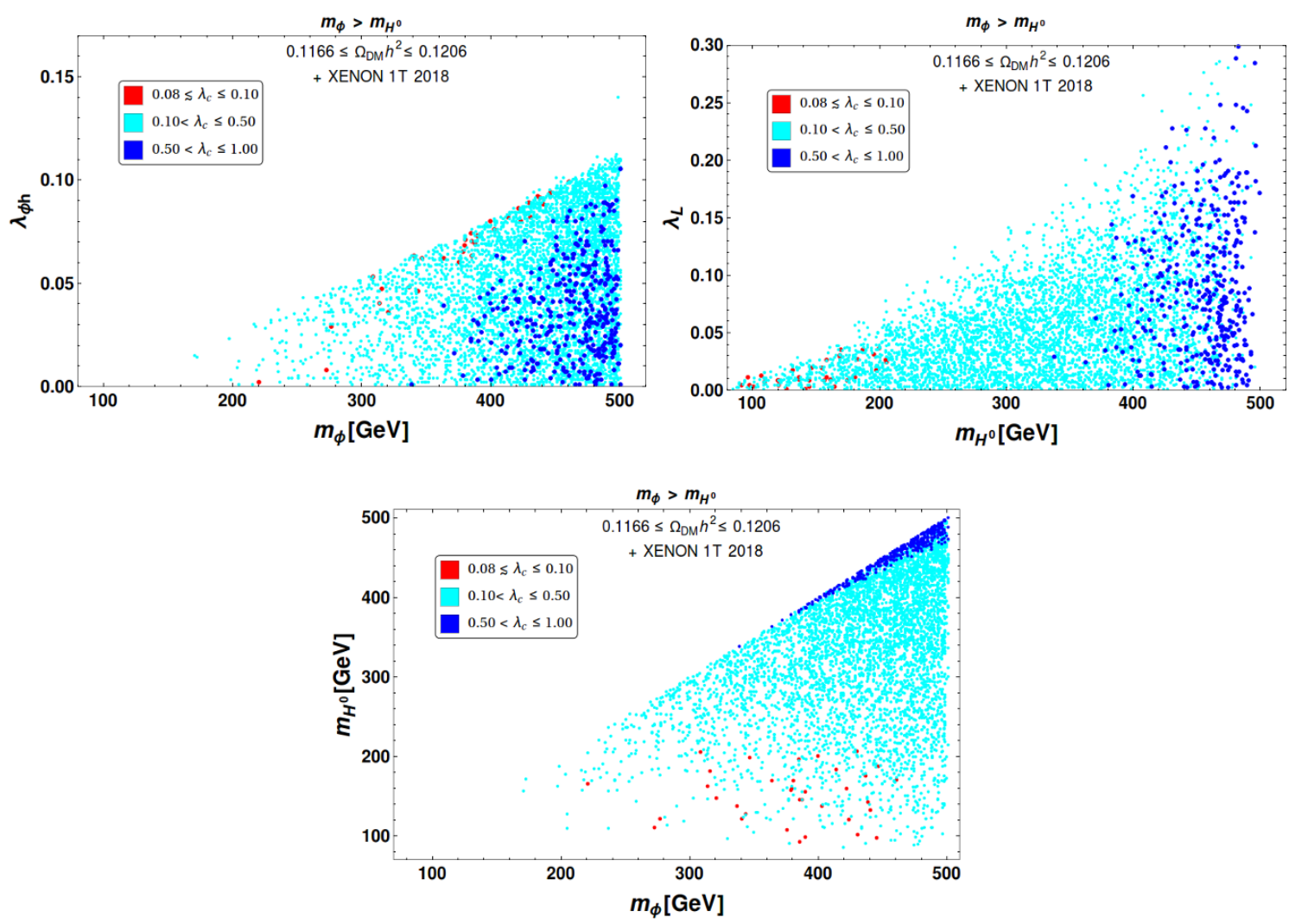

Figure 14. Relic Density and direct detection (XENON 1T 2018) at NLO allowed parameter space is shown in: $m_{\phi}-\lambda_{\phi h}$ plane (top left panel), $m_{H^{0}}-\lambda_{L}$ plane (top right panel) and $m_{\phi}-m_{H^{0}}$ plane (bottom panel). The scans are performed for the mass hierarchy $m_{\phi}>m_{H^{0}}$.

\section{Case II: $m_{\phi} \leq m_{H^{0}}$}

Naturally here the conversion of heavier $H^{0}$ to the lighter component $\phi$ will mainly dictate the relic density of DM components on top their annihilations to SM. Relic density allowed parameter space for $m_{\phi} \leq m_{H^{0}}$ is shown in figure 15. Again, this is illustrated in $m_{\phi}-\lambda_{\phi h}$ plane (top left), $m_{H^{0}}-\lambda_{L}$ plane (top right), $m_{\phi}-m_{H^{0}}$ plane (bottom left) and $\Omega_{\phi} h^{2} / \Omega_{\mathrm{DM}} h^{2}(\%)-\Omega_{H^{0}} h^{2} / \Omega_{\mathrm{DM}} h^{2}(\%)$ plane (bottom right). Different ranges of $\lambda_{c}$ are shown by the same colour code as in figure 12, 14. Let us first focus on the top left figure. It shows that for small values of $\lambda_{c}$, relic density allowed parameter space points lie in the vicinity of single component framework of $\phi$ (red points in figure). In absence of a lighter mode, the relic density of $\phi$ is essentially governed by its annihilation to SM and due to small conversion cross-section the production of $\phi$ is also not large enough to change the conclusion. However, the situation changes significantly with larger $\lambda_{c}$ (cyan and blue points), where we see again that the overabundant region of the single component scenario is getting allowed by relic density. In order to understand this let us remind ourself of the CBEQ for $m_{\phi}<m_{H^{0}}$ as depicted in eq. (5.10). In particular, the number density of $\phi$ is dictated by $\dot{n_{\phi}}+3 H n_{\phi} \simeq-\mathcal{F}_{\phi}+\mathcal{F}_{\Phi \phi}$. With larger $\lambda_{c}$ and larger conversion, $\mathcal{F}_{\Phi \phi}$ increases to reduce the effective $\mathcal{F}_{\phi}$ that determines the relic of $\phi$. Therefore, to keep the relic density of $\phi$ to cor- 

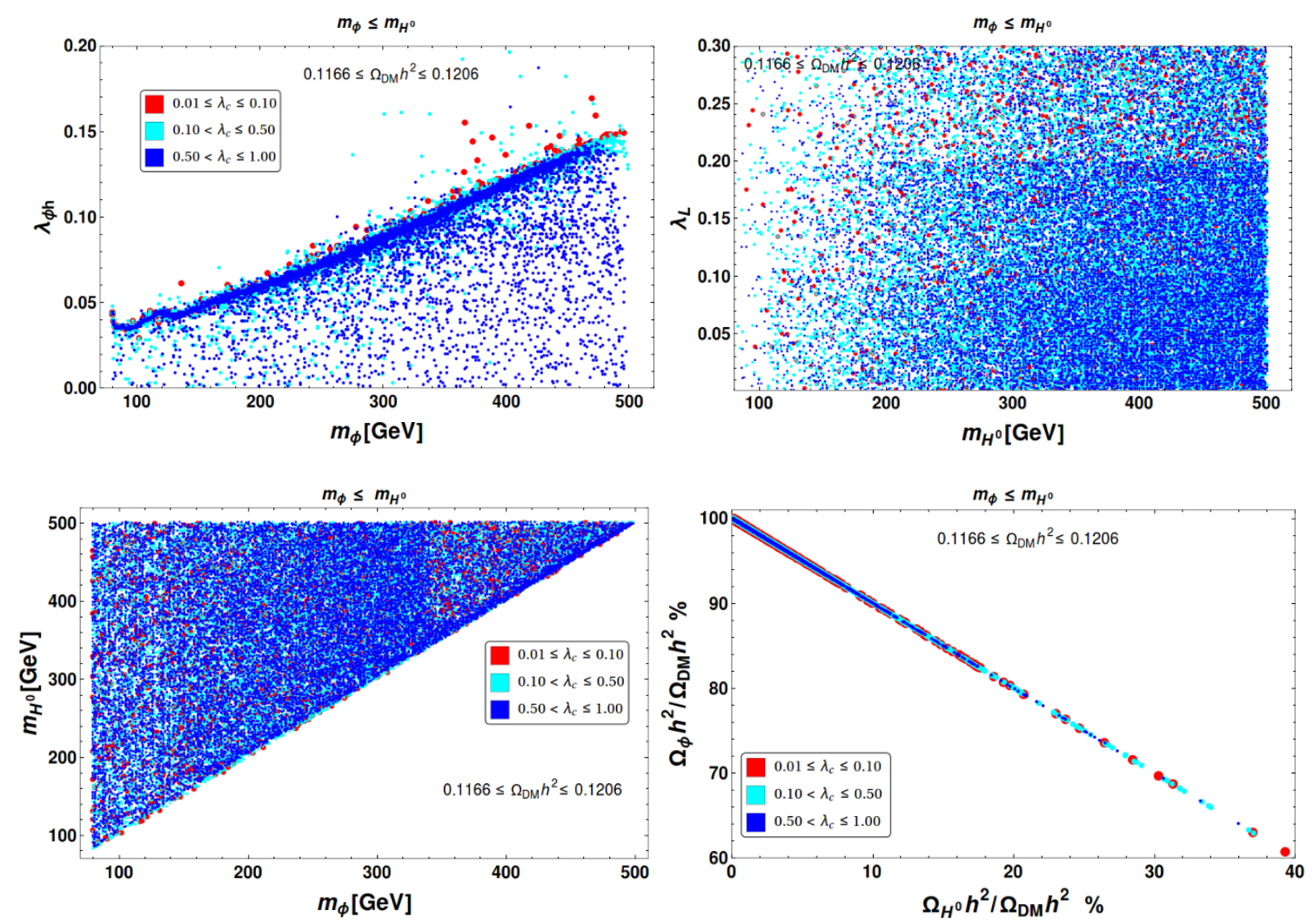

Figure 15. Relic Density allowed parameter space is shown in $m_{\phi}-\lambda_{\phi h}$ plane (top left), $m_{H^{0}}-\lambda_{L}$ plane (top right), $m_{\phi}-m_{H^{0}}$ plane (bottom left) and $\Omega_{\phi} h^{2} / \Omega_{\mathrm{DM}} h^{2}(\%)-\Omega_{H^{0}} h^{2} / \Omega_{\mathrm{DM}} h^{2}(\%)$ plane (bottom right) for the mass hierarchy $m_{\phi} \leq m_{H^{0}}$.

rect order, $\mathcal{F}_{\phi}$ has to increase. Now recall, $\mathcal{F}_{\phi}=\langle\sigma v\rangle_{\phi \phi \rightarrow \mathrm{SM} \mathrm{SM}}\left(n_{\phi}\right)^{2} \sim 1 /\langle\sigma v\rangle_{\phi \phi \rightarrow \mathrm{SM} \mathrm{SM}}$ as $n_{\phi} \sim 1 /\langle\sigma v\rangle_{\phi \phi \rightarrow \mathrm{SM} \text { SM}}$. Therefore, to increase $\mathcal{F}_{\phi}$, one has to reduce the annihilation cross-section $\langle\sigma v\rangle_{\phi \phi \rightarrow \mathrm{SM} \mathrm{SM}}$. This is possible by reducing $\lambda_{\phi h}$ as we see here in the plot. Next let us discuss the top right figure. This figure in $m_{H^{0}}-\lambda_{L}$ plane essentially depicts that with larger $\lambda_{c}$, larger $m_{H^{0}}$ is favoured to tame the DM conversion as well as annihilation cross-section to keep the relic within limit. The dependence however is not that much significant due to the presence of large number of co-annihilation channels which remain unaffected by $\lambda_{c}$. In the bottom left panel, mass correlation has been plotted and carries no information. Lastly, bottom right figure shows the relative relic density contributions of these two components. It is well understood that an additional channel for annihilation of $H^{0}$ only reduces the possibility of bringing $\Omega_{H^{0}} h^{2}$ in the correct ballpark due to already existing gauge mediated annihilation and co-annihilation channels. Therefore, for small $\lambda_{c}$, it is still possible to get a contribution from $\Omega_{H^{0}} h^{2} \sim 40 \%$, but that becomes harder with large $\lambda_{c}$, where the relic density contribution of $H^{0}$ is further limited to $\Omega_{H^{0}} h^{2} \sim 20 \%$.

Direct search constraints from XENON 1T 2018 on the relic density allowed points are shown in figure 16. To emphasise again, the demand of these plots are simultaneous satisfaction of XENON1T limit for both DM components. The main outcome of this plot is to see the absence of small $\lambda_{c}$ points (red dots) upto $\lambda_{c} \sim 0.30$. This is simply due to 

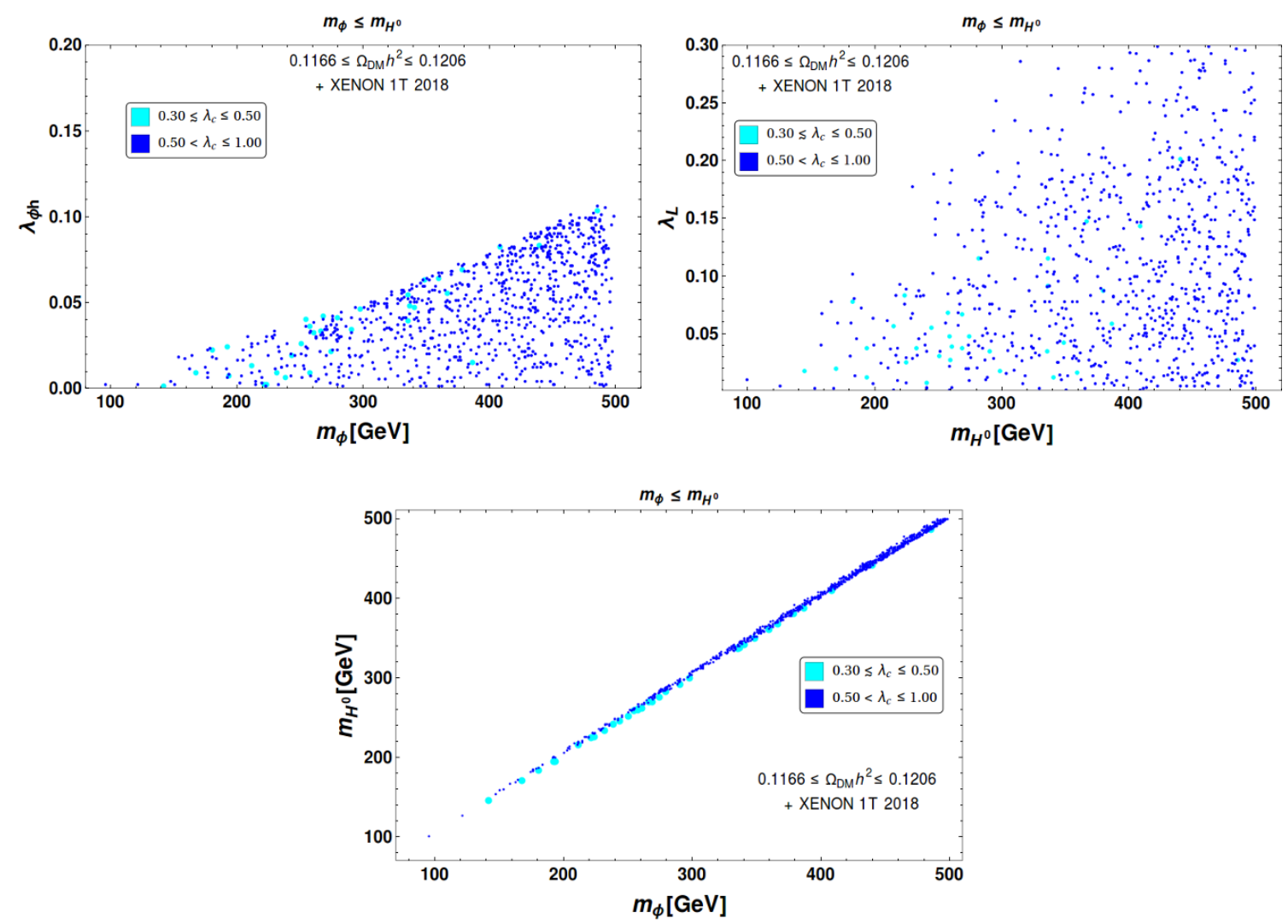

Figure 16. Relic Density and direct detection (XENON 1T 2018) at NLO allowed parameter space is shown in: $m_{\phi}-\lambda_{\phi h}$ plane (top left panel), $m_{H^{0}}-\lambda_{L}$ plane (top right panel) and $m_{\phi}-m_{H^{0}}$ plane (bottom panel). The scans are performed for the mass hierarchy $m_{\phi} \leq m_{H^{0}}$.

the fact that, with small $\lambda_{c}$, the required $\lambda_{\phi h}$ is high enough for $\phi$ DM to be discarded by XENON1T data. The other important feature is that with larger $\lambda_{c}$, larger DM masses are favoured. Lastly a very important conclusion comes from the bottom panel figure in the mass correlation plot. This shows, as only high $\lambda_{c}$ region is allowed, the conversion of $H^{0}$ to $\phi$ still needs to be restricted and therefore the mass difference between the two DM components $\left(m_{H^{0}}-m_{\phi}\right)$ has to be very small. These features are all distinct from that of $m_{\phi}>m_{H^{0}}$ region.

So far our discussion has been focused on DM mass region $m_{W^{ \pm}} \leq m_{H^{0}}, m_{\phi} \leq$ $500 \mathrm{GeV}$. But if $m_{H^{0}}<m_{W^{ \pm}}$or $m_{\phi}<m_{W^{ \pm}}$, while other DM mass is heavier than $m_{W^{ \pm}}$, the only region available for lighter DM with mass $<m_{W^{ \pm}}$are the Higgs and $Z$ resonance regions: $m_{H^{0}} \sim \frac{m_{Z}}{2}, \frac{m_{h}}{2}$ and $m_{\phi} \sim \frac{m_{h}}{2}$. It is important to remind that the resonance regions are already available in absence of second DM component and therefore brings no new phenomenological outcome.

\section{$7 \quad$ Indirect searches}

It is possible to probe DM signals in the context of different indirect detection experiments as well. The experiments look for astrophysical sources of SM particles produced through 
DM annihilations or via DM decays. Amongst these final states, photon and neutrinos, being neutral and stable can reach indirect detection probes without getting affected much by intermediate regions. For WIMP type DM, emitted photons lie in the gamma ray regime that can be measured at space-based telescopes like the Fermi-LAT or ground-based telescopes like MAGIC [104]. The photon flux in a specific energy range is written as

$$
\Phi_{F}=\frac{1}{4 \pi} \frac{\langle\sigma v\rangle_{\mathrm{ann}}}{2 m_{\mathrm{DM}}^{2}} \int_{E_{\min }}^{E_{\max }} \frac{d N_{\gamma}}{d E_{\gamma}} d E_{\gamma} \int_{\mathrm{LOS}} d x \rho^{2}(r(b, l, x)),
$$

where $r(b, l, x)$ is the distance of the DM halos from the galactic center. Galactic coordinates are defined by $b, l$ and $\rho(r)$ represents the DM density profile. Eq. (7.1) is further rewritten as

$$
\Phi_{F}=\frac{1}{4 \pi} \frac{\langle\sigma v\rangle_{\mathrm{ann}}}{2 m_{\mathrm{DM}}^{2}} \int_{E_{\min }}^{E_{\max }} \frac{d N_{\gamma}}{d E_{\gamma}} d E_{\gamma} \times J
$$

where $J=\int d x \rho^{2}(r(b, l, x))$ is conventionally known as $J$-factor and LOS means line of sight. Now from the observed Gamma ray flux produced due to DM annihilations, one can constrain the DM annihilation into different charged final states like $\mu^{+} \mu^{-}, \tau^{+} \tau^{-}$, $W^{+}, W^{-}$and $b^{+} b^{-}$. It is pertinent to mention here that along with the continuous gamma ray spectrum, gamma ray lines originated from IDM annihilations are also of special interest [105]. It has been shown in [106] that such a feature, characteristic of inert doublet dark matter (IDM) model, can indeed be important for DM mass above TeV where Sommerfeld enhancement becomes relevant. Since we mostly focus on the intermediate mass range of IDM in this study $(\sim 80-500 \mathrm{GeV})$, aforementioned constraint is extremely subdued.

In the multicomponent dark matter set up as we have here, it is expected that the $\gamma$ ray flux will be the sum of the contributions from both $\phi$ and $H^{0}$. Then we can write the total $\gamma$ ray flux can be written as:

$$
\Phi_{F}^{T}=\Phi_{F}^{\phi}+\Phi_{F}^{H^{0}}
$$

where $\Phi_{F}^{\phi}$ and $\Phi_{F}^{H^{0}}$ are the individual contribution to $\Phi_{F}^{T}$ from $\phi$ and $H^{0}$. Following eq. (7.2), it would be natural to write

$$
\frac{\langle\sigma v\rangle_{X X}^{\mathrm{eff}}}{m_{\mathrm{R}}^{2}} \rho_{T}^{2}=\frac{\langle\sigma v\rangle_{\phi \phi \rightarrow X X}}{m_{\phi}^{2}} \rho_{\phi}^{2}+\frac{\langle\sigma v\rangle_{H^{0} H^{0} \rightarrow X X}}{m_{H^{0}}^{2}} \rho_{H^{0}}^{2},
$$

where $X, X$ denote the annihilation products and $m_{R}$ indicates some effective mass scale. One can further write the effective annihilation cross-section in eq. (7.4) considering $m_{\mathrm{R}}$ to be the reduced mass of the two component system as

$$
\langle\sigma v\rangle_{X X}^{\text {eff }}=\left(\frac{\Omega_{H^{0}}}{\Omega_{T}}\right)^{2} \frac{m_{\phi}^{2}}{\left(m_{\phi}+m_{H^{0}}\right)^{2}}\langle\sigma v\rangle_{H^{0} H^{0} \rightarrow X X}+\left(\frac{\Omega_{\phi}}{\Omega_{T}}\right)^{2} \frac{m_{H^{0}}^{2}}{\left(m_{\phi}+m_{H^{0}}\right)^{2}}\langle\sigma v\rangle_{\phi \phi \rightarrow X X} .
$$

It turns out that for real singlet scalar dark matter indirect searches does not pose strong bound [18, 107]; while for single component IDM candidate, the indirect search rules out the region of $m_{H^{0}}<400 \mathrm{GeV}$ due to large thermal average cross section of $H^{0} H^{0} \rightarrow W^{+} W^{-}$channel [106-109]. Hence in our proposed two component DM set up 

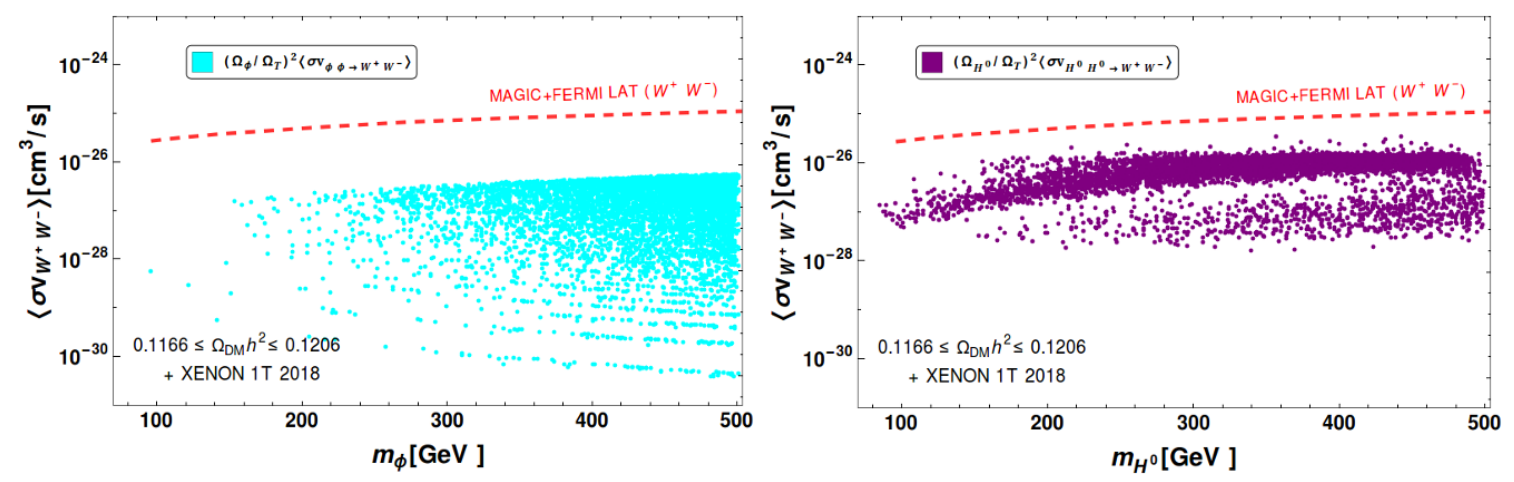

Figure 17. DM annihilations $\phi \phi \rightarrow W^{+} W^{-}$(left) and $H^{0} H^{0} \rightarrow W^{+} W^{-}$(right) normalised by branching fraction of individual relic densities compared against the latest indirect detection bounds of Fermi-LAT [104].

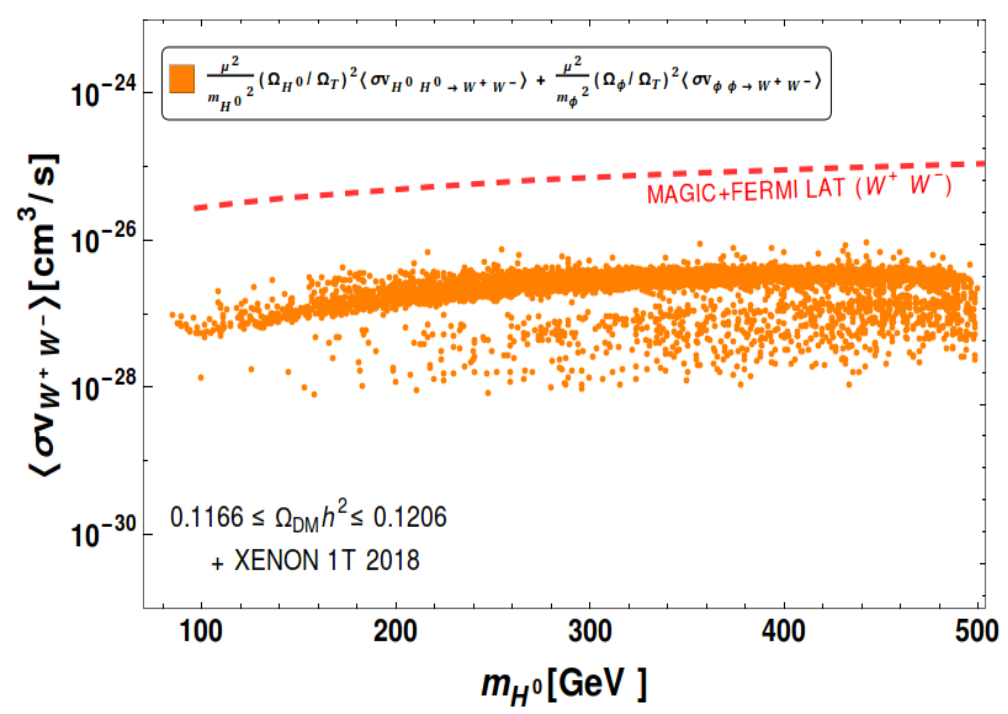

Figure 18. Effective DM annihilations to $W^{+} W^{-}$as defined in eq. (7.4) in the two-component model for relic density and direct search allowed points compared against the latest indirect detection bounds of Fermi-LAT [104].

we shall mostly focus on the $\langle\sigma v\rangle_{\mathrm{DM}, \mathrm{DM} \rightarrow \mathrm{W}^{+} \mathrm{W}^{-}}$and compare it with the indirect search experimental bound from Magcic+Fermi Lat bound [104]. In left panel of figure 17, we show the contribution of $\phi$ DM in $\langle\sigma v\rangle_{W^{+} W^{-}}^{\text {fff }}$ for all relic and SI DD cross section satisfied points and compare it with the limit coming from Fermi Lat experiment [104]. Similar comparison is drawn for IDM $\left(H^{0}\right)$ in right panel of figure 17. Finally in figure 18, we show the order of magnitude of total $\langle\sigma v\rangle_{W^{+} W^{-}}^{\text {eff }}$ for all relic density and SI DD (at NLO) satisfied points and find it to lie well below the upper limit imposed by Fermi Lat experiment. The relative abundance of individual relic densities and ratio of reduced mass to the DM mass helps to evade the indirect search bound on $\langle\sigma v\rangle_{\mathrm{DM}, \mathrm{DM} \rightarrow \mathrm{W}^{+} \mathrm{W}^{-}}$successfully. We have also confirmed that the bounds on $\langle\sigma v\rangle^{\text {eff }}$ for $b \bar{b}, \mu^{+} \mu^{-}, \tau^{+} \tau^{-}$and other annihilation final products from Magic+Fermi Lat experiment [104] are easily satisfied in our set up. 


\section{Electroweak vacuum stability and high scale perturbativity in presence of RH neutrino and DM}

One of the motivations of this work is to show that the presence of right handed neutrinos to yield correct neutrino masses in presence of multipartite DM. Although the neutrino sector considered here seems decoupled from the dark sector, is not completely true. The effect of the $\mathrm{RH}$ neutrinos alter the allowed DM parameter space when the model is validated at high scale. As already stated before, we employ type-I seesaw mechanism to generate the light neutrino mass, for which three $\mathrm{RH}$ neutrinos are included in the set up.

\subsection{Neutrino Yukawa couplings}

We first describe the strategy in order to study their impact on RG evolution. For simplicity, the $\mathrm{RH}$ neutrino mass matrix $M_{N}$ is considered to be diagonal with degenerate entries, i.e. $M_{N_{i=1,2,3}}=M_{R}$. It is to be noted that in the RG equation, $\operatorname{Tr}\left[Y_{\nu}^{\dagger} Y_{\nu}\right]$ enters. In order to extract the information on $Y_{\nu}$, we use the type-I seesaw formula for neutrino mass $m_{\nu}=Y_{\nu}^{T} Y_{\nu} \frac{v^{2}}{2 M_{R}}$. Then, naively one would expect that large Yukawa couplings are possible with even heavier RH neutrino masses. For example with $M_{R} \sim 10^{14} \mathrm{GeV}, Y_{\nu}$ comes out to be 0.3 in order to obtain $m_{\nu} \simeq 0.05 \mathrm{eV}$. However, contrary to this, it can be shown that even with smaller $M_{R}$ one can achieve large values of $\operatorname{Tr}\left[Y_{\nu}^{\dagger} Y_{\nu}\right]$, but effectively keeping $Y_{\nu}^{T} Y_{\nu}$ small, using a special flavor structure of $Y_{\nu}$ through Casas-Ibarra parametrization [58]. Note that our aim is to study the maximum effect coming from the right handed neutrino Yukawa, i.e. with large $\operatorname{Tr}\left[Y_{\nu}^{\dagger} Y_{\nu}\right]$, on EW vacuum stability. For this purpose, we use the parametrisation by [110] and write $Y_{\nu}$ as

$$
Y_{\nu}=\sqrt{2} \frac{\sqrt{M_{R}}}{v} \mathcal{R} \sqrt{m_{\nu}^{d}} U_{\mathrm{PMNS}}^{\dagger}
$$

where $m_{\nu}^{d}$ is the diagonal light neutrino mass matrix and $U_{\mathrm{PMNS}}$ is the unitary matrix diagonalizing the neutrino mass matrix $m_{\nu}$ such that $m_{\nu}=U_{\mathrm{PMNS}}^{*} m_{\nu}^{d} U_{\mathrm{PMNS}}^{\dagger}$. Here $\mathcal{R}$ represents a complex orthogonal matrix which can be written as $\mathcal{R}=\mathcal{O} \exp (i \mathcal{A})$ with $\mathcal{O}$ as real orthogonal matrix and $\mathcal{A}$ as real antisymmetric matrices. Using above parametrisation, then one gets,

$$
\operatorname{Tr}\left[Y_{\nu}^{\dagger} Y_{\nu}\right]=\frac{2 M_{R}}{v^{2}} \operatorname{Tr}\left[\sqrt{m_{\nu}^{d}} e^{2 i \mathcal{A}} \sqrt{m_{\nu}^{d}}\right]
$$

Note that the real antisymmetric matrix $\mathcal{A}$ however does not appear in the seesaw expression for neutrino mass as $m_{\nu}=\frac{Y_{\nu}^{T} Y_{\nu} v^{2}}{2 M_{R}}$. Therefore with any suitable choice of $\mathcal{A}$, it would actually be possible to have relatively large Yukawa even with light $M_{R}$.

\section{2 $\beta$ functions and RG running}

To study the high scale validity of this multi-component DM model with neutrino mass (including perturbativity and vacuum stability), we need to consider the RG running of the associated couplings. The framework contains few additional mass scales: one extra scalar singlet, one inert doublet and three RH neutrinos with mass $M_{N_{i=1,2,3}}\left(=M_{R}\right)$. Hence 
in the study of RG running, different couplings will enter into different mass scales. In DM phenomenology, we have considered the physical masses of DM sector particles within $\sim 500 \mathrm{GeV}$. Then for simplicity, we can safely ignore the small differences between the dark sector particle masses and identify the single additional mass scale as $m_{\mathrm{DM}}$. On the other hand, RH neutrinos are considered to be heavier than the scalars present in the model $\left(M_{R}>m_{\mathrm{DM}}\right)$. Hence, for running energy scale $\mu>m_{\mathrm{DM}}$, we need to consider the couplings associated to DM sector in addition to SM while for $\mu>M_{N_{i=1,2,3}}$, the neutrino couplings will additionally enter into the scenario. Below we provide the one loop $\beta$ functions for the additional for the couplings involved in our model, when $\mu>M_{R}$.

\section{$\beta$ functions of gauge couplings [111]}

$$
\begin{aligned}
& \beta_{g_{1}}=\frac{21}{5} g_{1}^{3}, \\
& \beta_{g_{2}}=-3 g_{2}^{3}, \\
& \beta_{g_{3}}=-7 g_{3}^{3} .
\end{aligned}
$$

$\beta$ functions of Yukawa couplings $[111,112]$ :

$$
\begin{aligned}
\beta_{y_{t}} & =\frac{3}{2} y_{t}^{3}+y_{t}\left(3 y_{t}^{2}-8 g_{3}^{2}-\frac{17}{20} g_{1}^{2}-\frac{9}{4} g_{2}^{2}+y_{t} \operatorname{Tr}\left[Y_{\nu}^{\dagger} Y_{\nu}\right]\right), \\
\beta_{\operatorname{Tr}\left[Y_{\nu}^{\dagger} Y_{\nu}\right]} & =3 \operatorname{Tr}\left[\left(Y_{\nu}^{\dagger} Y_{\nu}\right)^{2}\right]+\operatorname{Tr}\left[Y_{\nu}^{\dagger} Y_{\nu}\right]\left(-\frac{9}{10} g_{1}^{2}-\frac{9}{2} g_{2}^{2}+6 y_{t}^{2}+2 \operatorname{Tr}\left[Y_{\nu}^{\dagger} Y_{\nu}\right]\right) .
\end{aligned}
$$

$\beta$ functions of quartic scalar couplings [111]

$$
\begin{aligned}
\beta_{\lambda_{H}}= & \frac{27}{200} g_{1}^{4}+\frac{9}{20} g_{1}^{2} g_{2}^{2}+\frac{9}{8} g_{2}^{4}-\frac{9}{5} g_{1}^{2} \lambda_{H}-9 g_{2}^{2} \lambda_{H}+24 \lambda_{H}^{2}+12 \lambda_{H} y_{t}^{2}-6 y_{t}^{4} \\
& +2 \lambda_{1}^{2}+2 \lambda_{1} \lambda_{2}+\lambda_{2}^{2}+\lambda_{3}^{2}+\frac{1}{2} \lambda_{\phi h}^{2}+4 \lambda_{H} \operatorname{Tr}\left[Y_{\nu}^{\dagger} Y_{\nu}\right]-2 \operatorname{Tr}\left[\left(Y_{\nu}^{\dagger} Y_{\nu}\right)^{2}\right] \\
\beta_{\lambda_{3}}= & -\frac{9}{5} g_{1}^{2} \lambda_{3}-9 g_{2}^{2} \lambda_{3}+8 \lambda_{1} \lambda_{3}+12 \lambda_{2} \lambda_{3}+4 \lambda_{3} \lambda_{H}+4 \lambda_{3} \lambda_{\Phi}+6 \lambda_{3} y_{t}^{2}+2 \lambda_{3} \operatorname{Tr}\left[Y_{\nu}^{\dagger} Y_{\nu}\right], \\
\beta_{\lambda_{2}}= & +\frac{9}{5} g_{1}^{2} g_{2}^{2}-\frac{9}{5} g_{1}^{2} \lambda_{2}-9 g_{2}^{2} \lambda_{2}+8 \lambda_{1} \lambda_{2}+4 \lambda_{2}^{2}+8 \lambda_{3}^{2}+4 \lambda_{2} \lambda_{H}+4 \lambda_{2} \lambda_{\Phi}+6 \lambda_{2} y_{t}^{2}+2 \lambda_{2} \operatorname{Tr}\left[Y_{\nu}^{\dagger} Y_{\nu}\right], \\
\beta_{\lambda_{1}=}= & \frac{27}{100} g_{1}^{4}-\frac{9}{10} g_{1}^{2} g_{2}^{2}+\frac{9}{4} g_{2}^{4}-\frac{9}{5} g_{1}^{2} \lambda_{1}-9 g_{2}^{2} \lambda_{1}+4 \lambda_{1}^{2}+2 \lambda_{2}^{2}+2 \lambda_{3}^{2}+12 \lambda_{1} \lambda_{H} \\
& +4 \lambda_{2} \lambda_{H}+12 \lambda_{1} \lambda_{\Phi}+4 \lambda_{2} \lambda_{\Phi}+6 \lambda_{1} y_{t}^{2}+\lambda_{c} \lambda_{\phi h}+2 \lambda_{1} \operatorname{Tr}\left[Y_{\nu}^{\dagger} Y_{\nu}\right], \\
\beta_{\lambda_{\Phi}=}= & 24 \lambda_{\Phi}^{2}+2 \lambda_{1}^{2}+2 \lambda_{1} \lambda_{2}-9 g_{2}^{2} \lambda_{\Phi}+\frac{27}{200} g_{1}^{4}+\frac{9}{20} g_{1}^{2}\left(-4 \lambda_{\Phi}+g_{2}^{2}\right)+\frac{9}{8} g_{2}^{4}+\lambda_{2}^{2}+\lambda_{3}^{2}+\frac{1}{2} \lambda_{c}^{2}, \\
\beta_{\lambda_{\phi h}=}= & \frac{9}{10} g_{1}^{2} \lambda_{\phi h}-\frac{9}{2} g_{2}^{2} \lambda_{\phi h}+4 \lambda_{\phi h}^{2}+12 \lambda_{\phi h} \lambda_{H}+\lambda_{\phi h} \lambda_{\phi}+6 \lambda_{\phi h} y_{t}^{2} \\
& +4 \lambda_{1} \lambda_{c}+2 \lambda_{2} \lambda_{c}+2 \lambda_{\phi h} T r\left[Y_{\nu}^{\dagger} Y_{\nu}\right], \\
\beta_{\lambda_{c}=} & -\frac{9}{10} g_{1}^{2} \lambda_{c}-\frac{9}{2} g_{2}^{2} \lambda_{c}+12 \lambda_{c} \lambda_{\Phi}+4 \lambda_{1} \lambda_{\phi h}+2 \lambda_{2} \lambda_{\phi h}+\lambda_{c} \lambda_{\Phi}+4 \lambda_{c}^{2}, \\
\beta_{\lambda_{\phi}=}= & 3\left(4 \lambda_{c}^{2}+4 \lambda_{\phi h}^{2}+\lambda_{\phi}^{2}\right) .
\end{aligned}
$$

The above $\beta$ functions are generated using the model implementation in the code SARAH [113]. The running of $\lambda_{H}$ as in eq. (8.5) is influenced adversely mostly by top Yukawa 
coupling $y_{t} \sim \mathcal{O}(1)$ and right handed neutrino Yukawa coupling as $\operatorname{Tr}\left[Y_{\nu}^{\dagger} Y_{\nu}\right]$. On the other hand, multiparticle scalar DM couplings present in the model help in compensating the strong negative effect from $y_{t}$ and $\operatorname{Tr}\left[Y_{\nu}^{\dagger} Y_{\nu}\right]$. To evaluate $\operatorname{Tr}\left[Y_{\nu}^{\dagger} Y_{\nu}\right]$, we employ eq. (8.2). As an example, let us consider magnitude of all the entries of $\mathcal{A}$ to be equal, say $a$ with all diagonal entries to be zero. Then using the best fit values of neutrino mixing angles and cosnidering the mass of lightest neutrino zero, one can find for $M_{R}=1 \mathrm{TeV}, \operatorname{Tr}\left[Y_{\nu}^{\dagger} Y_{\nu}\right]$ can be as large as 1 with $a=8.1[110,114]$. Equipped with all these $\beta$ functions and strategy to estimate the relevant couplings present in them, let us now analyse the SM Higgs vacuum stability at high energy scale. Below we provide the stability and metastability criteria.

Stability criteria. The stability of Higgs vacuum can be ensured by the condition $\lambda_{H}>$ 0 at any scale. However, we have multiple scalars (SM Higgs doublet, one inert doublet and one gauge real singlet) in the model. Therefore we should ensure the boundedness or stability of the entire scalar potential in any field direction. This can be guaranteed by using the co-positivity criterion in eq. (3.1). Note that once $\lambda_{H}$ becomes negative the other copositivity conditions no longer remain valid.

Metastability criteria. On the other hand, when $\lambda_{H}$ becomes negative, there may exist another deeper minimum other than the EW one. Then the estimate of the tunnelling probability $\mathcal{P}_{T}$ of the EW vacuum to the second minimum is essential to confirm the metastability of the Higgs vacuum. Obviously, the Universe can be in a metastable state only, provided the decay time of the EW vacuum is longer than the age of the Universe. The tunnelling probability is given by $[4,5]$,

$$
\mathcal{P}_{T}=T_{U}^{4} \mu_{B}^{4} e^{-\frac{8 \pi^{2}}{3\left|\lambda_{H}\left(\mu_{B}\right)\right|}},
$$

where $T_{U}$ is the age of the Universe, $\mu_{B}$ is the scale at which tunnelling probability is maximized, determined from $\beta_{\lambda_{H}}=0$. Therefore, solving above equation, we see that metastable Universe requires $[4,5]$ :

$$
\lambda_{H}\left(\mu_{B}\right)>\frac{-0.065}{1-\ln \left(\frac{v}{\mu_{B}}\right)} .
$$

As noted in [4], for $\mu_{B}>M_{P}$ (Planck Scale, $M_{P}=1.22 \times 10^{19} \mathrm{GeV}$ ), one can safely consider $\lambda_{H}\left(\mu_{B}\right)=\lambda_{H}\left(M_{P}\right)$. One should also note, that even with meatstability of Higgs vacuum, the instability in other field direction may also occur. The conditions to avoid the possible instability along various field directions for $\lambda_{H}<0$ are listed below [115]:

(i) $\lambda_{\Phi}>0$ to avoid the unboundedness of the potential along $H^{0}, A^{0}$ and $H^{ \pm}$directions,

(ii) $\lambda_{1}>0$ to ensure the stability along a direction between $H^{ \pm}$and $h$,

(iii) $\lambda_{L}>0$ to ensure the stability along a direction between $H^{0}$ and $h$,

(iv) $\lambda_{S}>0$, to avoid unboundedness of potential along a direction between $A^{0}$ and $h$,

(v) $\lambda_{\phi}>0$, otherwise the potential will be unbounded along $\phi$ direction,

(vi) $\lambda_{\phi h}>0$, to ensure the stability along a direction between $\phi$ and $h$. 


\begin{tabular}{|c|c|c|c|c|c|c|c|c|c|}
\hline BPs & $m_{H^{0}}$ & $m_{\phi}$ & $m_{A^{0}}$ & $m_{H^{ \pm}}$ & $\lambda_{L}$ & $\lambda_{\phi h}$ & $\lambda_{c}$ & $\Omega_{H^{0}} h^{2}$ & $\Omega_{\phi} h^{2}$ \\
\hline BP1 & 330 & 343 & 333 & 339 & 0.043 & 0.065 & 0.2 & 0.033 & 0.086 \\
\hline BP2 & 427 & 449 & 438 & 440 & 0.086 & 0.017 & 0.3 & 0.027 & 0.088 \\
\hline
\end{tabular}

Table 2. Benchmark points used to analyse EW vacuum stability in our model. All masses are in GeVs. The other couplings used in these benchmark points play an important role; they are: BP1: $\left\{\lambda_{1}=0.285, \lambda_{2}=-0.17, \lambda_{3}=-0.033\right\}, \mathrm{BP} 2:\left\{\lambda_{1}=0.544, \lambda_{2}=-0.215, \lambda_{3}=-0.157\right\}$.

\begin{tabular}{|c|c|c|c|}
\hline BPs & $\Delta S\left(10^{-4}\right)$ & $\Delta T\left(10^{-4}\right)$ & $\mu_{\gamma \gamma}\left(10^{-5}\right)$ \\
\hline BP1 & -12 & 5.1 & 3 \\
\hline BP2 & -9 & 2.5 & 3 \\
\hline
\end{tabular}

Table 3. Estimate of electroweak precision parameters and $\mu_{\gamma \gamma}$ for the two benchmark points as chosen in table 2.

Now to begin the vacuum stability analysis in the present multi-component DM scenario, we first choose two benchmark values of DM masses $\left(\phi\right.$ and $\left.H^{0}\right)$ which satisfy both the relic density and direct detection bounds. These along with corresponding values of other relevant parameters are denoted by the set of two benchmark points, BP1 and BP2, as tabulated in table 2. These parameters are mentioned in the caption of table 2 for both benchmark points. We also show the value of electroweak parameters and $\mu_{\gamma \gamma}$ for these two benchmark points in table 3. We run the two loops RG equations for all the SM couplings and one loop RG equations for the other relevant couplings in the model from $\mu=m_{t}$ to $M_{P}$ energy scale. We use the inital boundary values of all the SM couplings as provided in [4]. The boundary values have been evaluated at $\mu=m_{t}$ in [4] by taking various threshold corrections and mismatch between top pole mass and $\overline{M S}$ renormalised couplings into account.

In figure 19, we show the running of $\lambda_{H}$ for BP1 as a function of energy scale $\mu$. The left panel shows running of $\lambda_{H}$ for different values of RH neutrino mass $M_{R}$, considering top quark mass $m_{t}=173.1 \mathrm{GeV}$, Higgs mass $m_{h}=125.09 \mathrm{GeV}$ and $\operatorname{Tr}\left[Y_{\nu}^{\dagger} Y_{\nu}\right]=0.5$. As it is visible that for low value of $M_{R} \sim 10^{4} \mathrm{GeV}, \lambda_{H}$ enters into unstable region at very early stage of its evolution (blue line in the figure). This has happened as the scalar couplings in $\beta_{\lambda_{H}}$ (eq. (8.5)) are not sufficiently large to counter the strong negative impact of $\operatorname{Tr}\left[Y_{\nu}^{\dagger} Y_{\nu}\right]$ which brings down the $\lambda_{H}$ shraply towards unstable region. On contrary, for large value of $M_{R} \sim 10^{14} \mathrm{GeV}$, the effect of $Y_{\nu}$ in $\beta_{\lambda_{H}}$ starts at a comparatively larger energy scale than the earlier case. As a consequence, although $\lambda_{H}$ becomes negative at some high energy scale, it stays in metastable region till $M_{P}$ energy scale (violate line). Green region here describes stable, white region describes metastable (see eq. (8.8)) and the red region indicates unstable solution for the potential. For comparison, we also display the evolution of $\lambda_{H}$ (black dotted curve) in absence of RH neutrinos in the theory with the inclusion of scalar couplings (related to DM) only. This clearly shows that in absence of RH neutrinos, EW vacuum could be absolutely stable till $M_{P}$ energy scale. In right panel of figure 19, we study the evolution of $\lambda_{H}$ considering $2 \sigma$ uncertainty of measured top mass $m_{t}$, keeping 

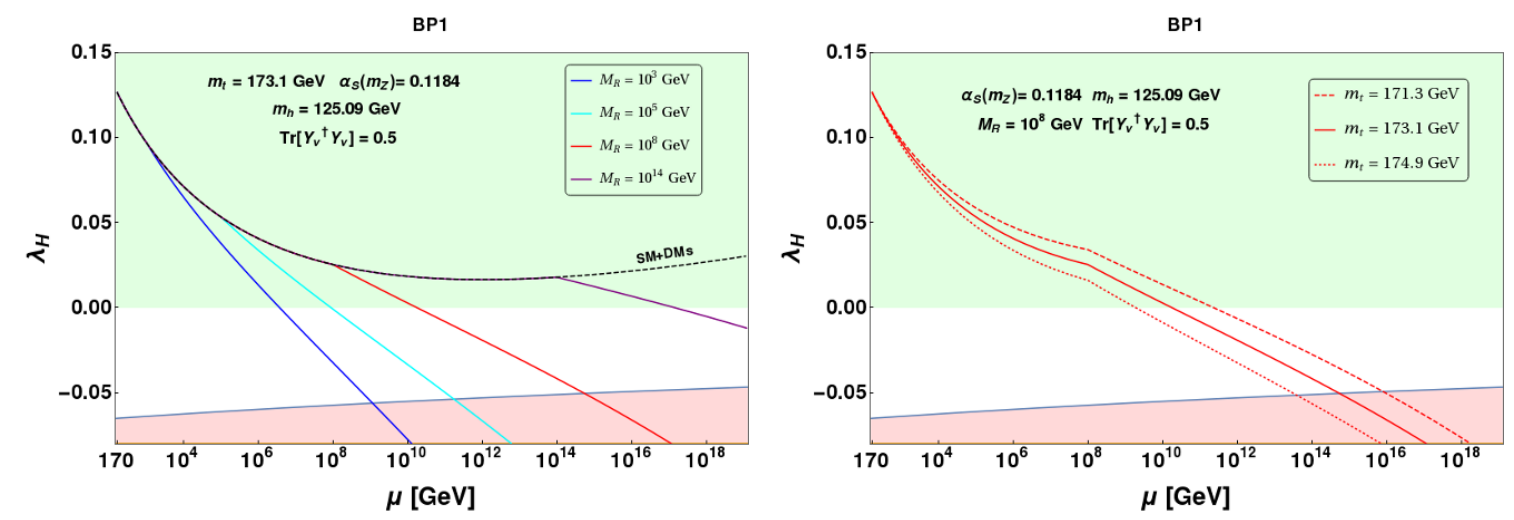

Figure 19. RG running of $\lambda_{H}$ with energy scale for BP1. In left panel, we have shown the effect of different right handed neutrinos masses, $M_{R}$ (indicated by different colours and mentioned in figure inset) for a fixed top quark mass $m_{t}=173.1 \mathrm{GeV}$. The black dotted line corresponds to the case when right handed neutrinos are absent in the scenario. In right panel, we choose a specific $M_{R}=10^{8} \mathrm{GeV}$ and consider top mass in $2 \sigma$ range: $m_{t}=173.1 \pm 0.9 \mathrm{GeV} \cdot \operatorname{Tr}\left[Y_{\nu}^{\dagger} Y_{\nu}\right]=0.5$ is kept constant in both plots.
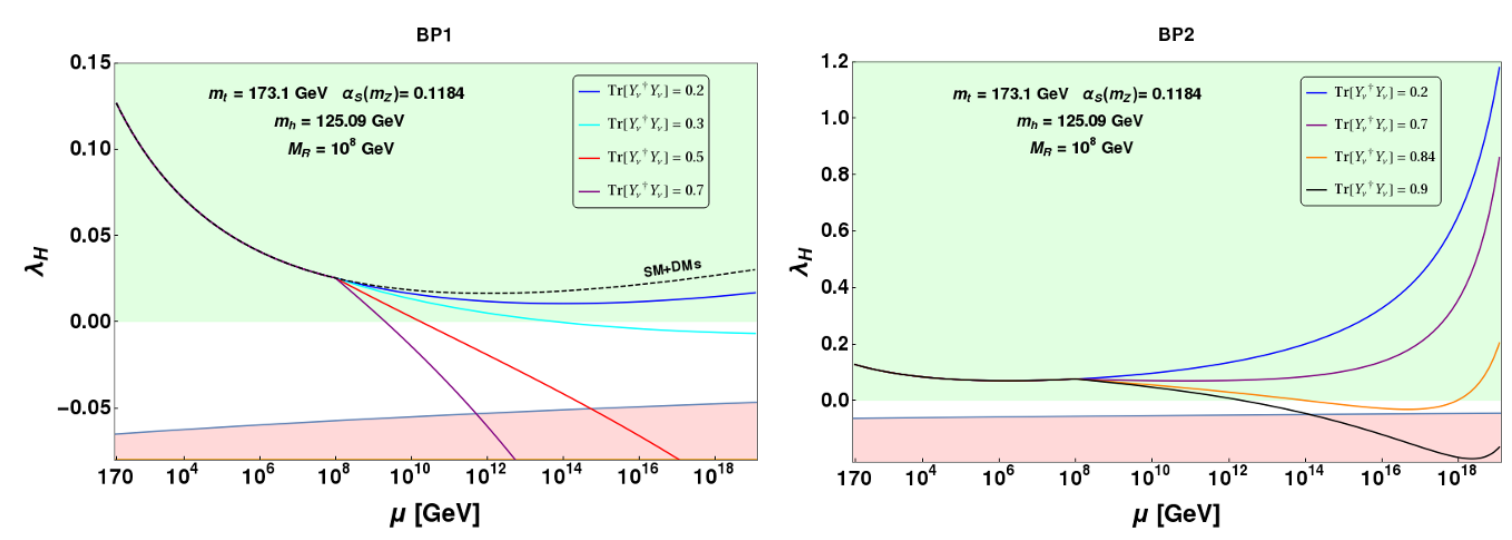

Figure 20. RG running of $\lambda_{H}$ with energy scale $\mu$ for different values of $\operatorname{Tr}\left[Y_{\nu}^{\dagger} Y_{\nu}\right]$ (shown in different colours and values are mentioned in the figure inset) for the benchmark points BP1 (left) and BP2 (right). Here we have chosen $M_{R}=10^{8} \mathrm{GeV}$ for illustration. The black dotted line here corresponds to the case when right handed neutrinos are absent in this scenario.

$m_{h}=125.09 \mathrm{GeV}, M_{R}=10^{8} \mathrm{GeV}$ and $\operatorname{Tr}\left[Y_{\nu}^{\dagger} Y_{\nu}\right]=0.5$ fixed. It is trivial to find that with the increase of top mass, $\lambda_{H}$ crosses zero at earlier stage in its evolution.

With comparatively lesser value of $\operatorname{Tr}\left[Y_{\nu}^{\dagger} Y_{\nu}\right] \sim 0.3$, the EW vacuum might be in metastable state provided other conditions $((i)-(v i))$ are satisfied as shown in left panel of figure 21. If we further reduce the value of $\operatorname{Tr}\left[Y_{\nu}^{\dagger} Y_{\nu}\right] \sim 0.2$ the EW vacuum might be absolutely stable. For the stability case we also show the satisfaction of all the copositivity criterias (eq. (3.1)) in left panel of figure 22. This ensures the boundness of the scalar potential in any arbitary field direction. The analysis for BP2 turns out to be similar as observed from right panels of figure 20-22. Note that due to comparatively larger values of the scalar couplings in BP2, we achieve a better results in stability point of view than BP1. 

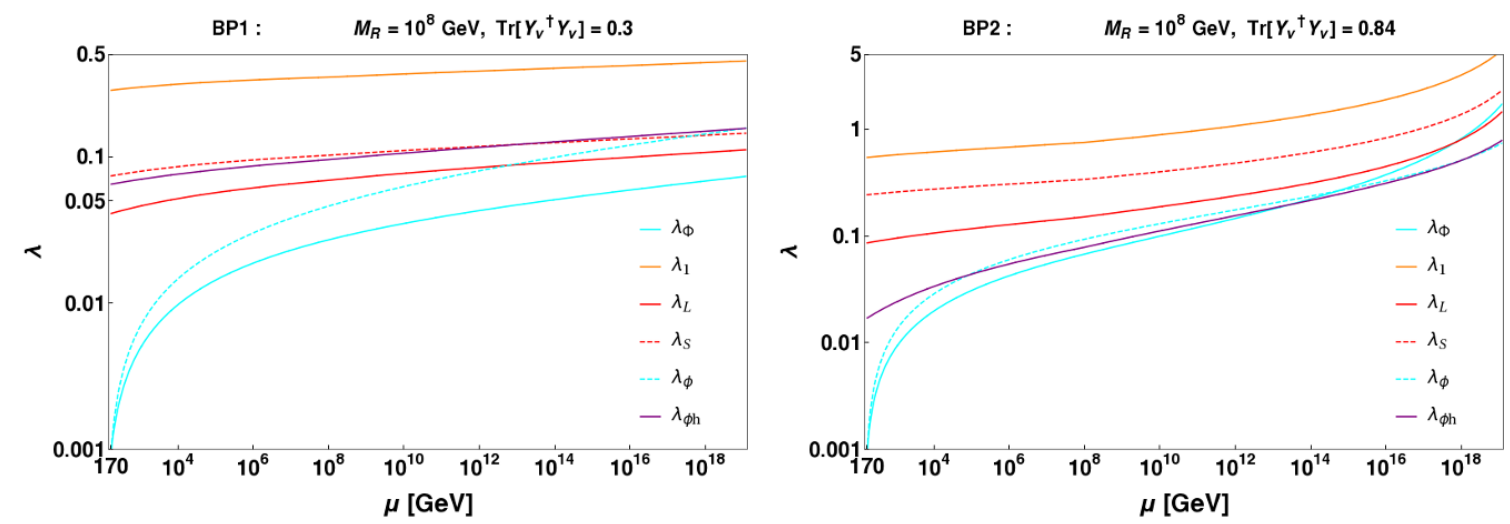

Figure 21. RG running of all the quartic couplings in metastability criteria for BP1 (left) and BP2 (right) to ensure the boundedness of the scalar potential in various field directions with energy scale $\mu$ for $\operatorname{Tr}\left[Y_{\nu}^{\dagger} Y_{\nu}\right]=0.3$ (left) and 0.84 (right). The choices of $\operatorname{Tr}\left[Y_{\nu}^{\dagger} Y_{\nu}\right]$ are demonstrated in cyan $(0.3)$ and in orange (0.84) in left and right panels of figure 20 to yield metastability.
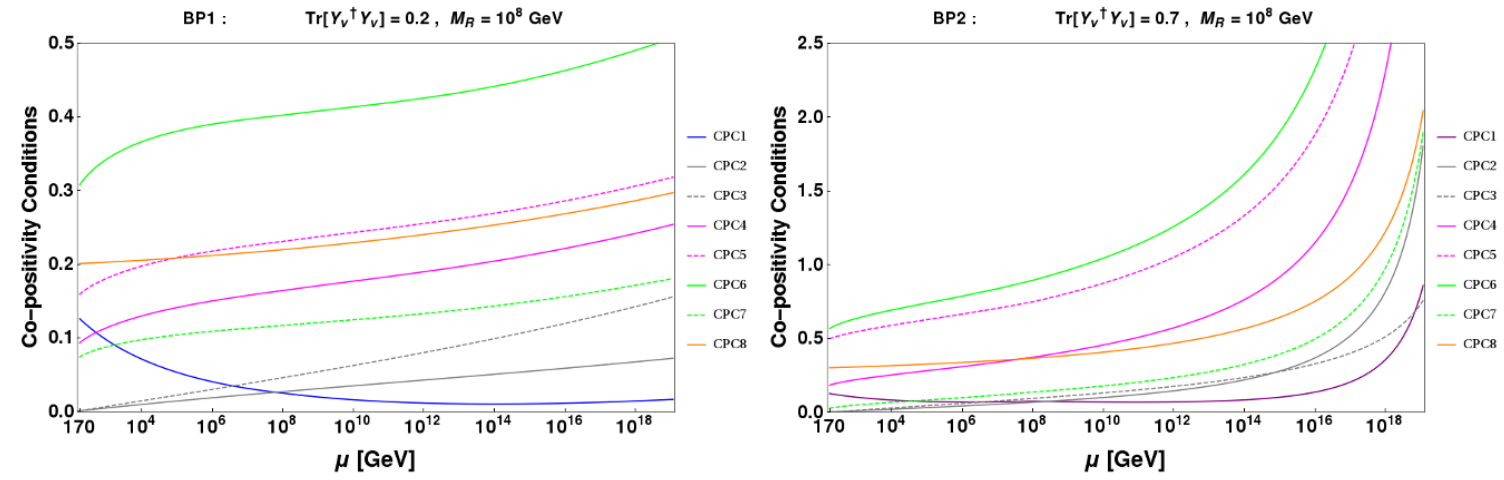

Figure 22. RG running of all copositivity criteria in eq. (3.1) for BP1 (left) and BP2 (right) to ensure the boundedness of the scalar potential in any field direction with energy scale $\mu$ for $\operatorname{Tr}\left[Y_{\nu}^{\dagger} Y_{\nu}\right]=0.2$ (left) and 0.7 (right) The choices of $\operatorname{Tr}\left[Y_{\nu}^{\dagger} Y_{\nu}\right]$ are shown in dark blue and purple colours in left and right panels of figure 20 respectively to yield stability.

Next we show the effect of $\operatorname{Tr}\left[Y_{\nu}^{\dagger} Y_{\nu}\right]$ in the RG evolution of $\lambda_{H}$ in figure 20 for BP1 (left) and BP2 (right). For the purpose we fix the RH neutrino mass scale $M_{R}=10^{8} \mathrm{GeV}$. It can be seen from left panel that large value of $\operatorname{Tr}\left[Y_{\nu}^{\dagger} Y_{\nu}\right] \sim 0.7$ brings down $\lambda_{H}$ towards the negative values at earlier energy scale. This is obvious from the $\beta$ function of $\lambda_{H}$ as the amount of scalar couplings for BP1 are not that effective in presence of such large value of $\operatorname{Tr}\left[Y_{\nu}^{\dagger} Y_{\nu}\right]$

Based on the inputs from above analysis, now we constrain $\operatorname{Tr}\left[Y_{\nu}^{\dagger} Y_{\nu}\right]-M_{R}$ parameter space using the stability, metastability and instability criteria (green, white and red regions respectively) for BP1 (left panel) and BP2 (right panel) in figure 23. The criteria has been set at Planck scale $\left(M_{P}\right)$. We use $\alpha_{S}=0.1184$ and $m_{h}=125.09 \mathrm{GeV}$ for both the plots. The solid lines indicate the contour for $m_{t}=173.1 \mathrm{GeV}$ while the dotted lines denote $2 \sigma$ uncertainty of the measured value of $m_{t}$. It can be concluded from figure 23 , that to have a stable/metastable EW vacuum, smaller values of $M_{R}$ requires low $\operatorname{Tr}\left[Y_{\nu}^{\dagger} Y_{\nu}\right]$ and vice versa. 

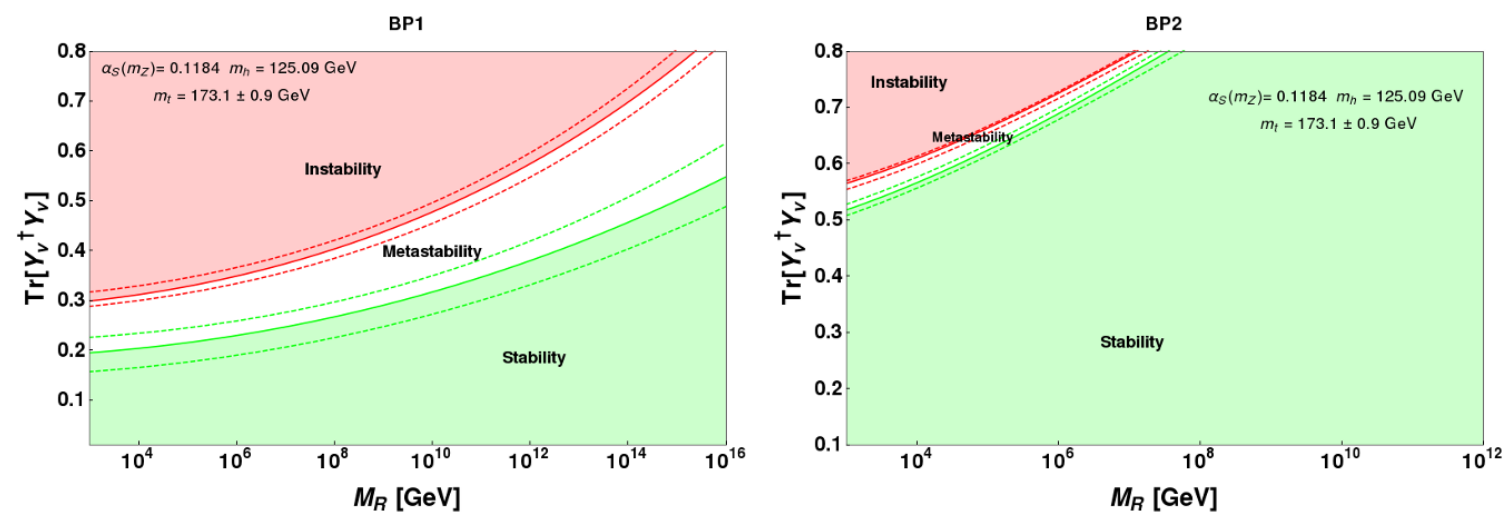

Figure 23. Stability, metastability and instability regions plot on $M_{R}-\operatorname{Tr}\left[Y_{\nu}^{\dagger} Y_{\nu}\right]$ plane for the benchmark point BP1 (left panel) and BP2 (right panel). For illustration we have considered top mass variation in $1 \sigma$ range: $173.1 \pm 0.9 \mathrm{GeV}$.
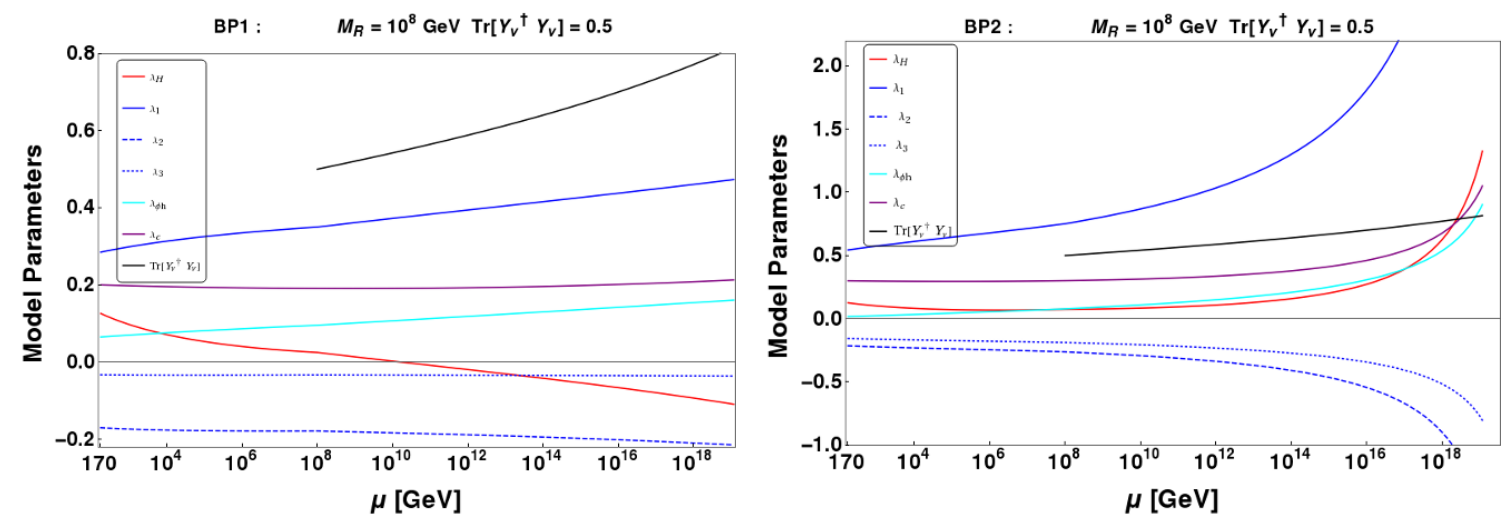

Figure 24. RG running of relevant coupling parameters for BP1 (left panel) and BP2 (right panel). $M_{R}=10^{8} \mathrm{GeV}, \operatorname{Tr}\left[Y_{\nu}^{\dagger} Y_{\nu}\right]=0.5$ and $m_{t}=173.1 \mathrm{GeV}$ have been kept fixed in both plots.

One important distinction between the left and right panel figures, corresponding to two different benchmark points, is that BP2 has significantly larger parameter space available from high scale stability. This is because of the larger values of $\lambda_{1,2,3}$ parameters used in BP2 compared to BP1 (see table 4 for details). Therefore, it can be concluded, that larger is the mass splitting in IDM sector, the more favourable it is from the stability point of view. However there is an important catch to this statement, which we will illustrate next.

In figure 24 we plot the running of all the individual couplings present in the set up. We see that (fortunately) for the two benchmark points used in the analysis, we are still okay with the perturbative limit at the high scale, i.e. all the couplings obey the perturbative limit, $\left|\lambda_{i}\right|<4 \pi,\left|\operatorname{Tr}\left[Y_{\nu}^{\dagger} Y_{\nu}\right]\right|<4 \pi$ at Planck scale. However, for BP1, with $M_{R}=10^{8} \mathrm{GeV}$, and $\operatorname{Tr}\left[Y_{\nu}^{\dagger} Y_{\nu}\right]=0.5$ as shown in the left panel yields unstable solution to EW vacuum with $\lambda_{H}$ running negative. On the other hand, BP2 with same choices of right handed neutrino mass and Yukawa yields a stable vacuum. Therefore, once again we find that larger splitting in IDM sector is more favourable for EW vacuum stability, as we have also inferred before from figure 23. But, it turns out that as larger splitting in the IDM sector also uses 
larger values of $\lambda_{1,2,3}$, it is possible, that many of those points become non-perturbative i.e. $\left|\lambda_{i}\right|>4 \pi$ when run upto Planck scale. We will show in the next section, that this very phenomena disallows many of the relic density and direct search allowed parameter space of the model. Another point to end this section is to note that our available parameter space from DM constraints heavily depend on large DM-DM conversion, which naturally comes from large choices of the conversion coupling $\lambda_{c}$. It is natural, that some of those cases will also be discarded by the perturbative limit $\left|\lambda_{c}\right|<4 \pi$, when we evaluate the validity of the model at high scale.

\subsection{Allowed parameter space of the model from high scale validity}

Finally, we come to the point where we can assimilate all the constraints together, from DM constraints to high scale validity. In order to do that, we choose relic density and direct search at NLO allowed parameter space of the model as discussed in section 6 and impose the high scale validity of the model till some energy scale $\mu$ by demanding:

- Stability of the scalar potential (eq. (3.1)) determined by satisfying copositivity conditions for any energy scale $\mu$.

- Non-violation of perturbativity and unitarity of all the relevant couplings present in the model as defined in eqs. (3.4) and (3.5).

Note that the high scale validity of the models does not depend on the structure of mass hierarchy of the DM candidates (i.e. on the sign of mass difference $m_{H^{0}}-m_{\phi}$ ). To study the EW vacuum stability we demand the positivity of $\lambda_{H}$ at each energy scale from EW to high scale $\mu$. As evident from $\beta_{\lambda_{H}}$ in eq. (8.5), a particular combination of the scalar couplings in the form of $2 \lambda_{1}^{2}+\lambda_{2}^{2}+\lambda_{3}^{2}+2 \lambda_{1} \lambda_{2}+\frac{1}{2} \lambda_{\phi h}^{2}$ determines the positivity of $\lambda_{H}$ during its running. However the factor $\lambda_{\phi h} \lesssim 0.1$ is strongly constrained from the SI DD cross section bound for $m_{\phi}<500 \mathrm{GeV}$. Hence, we can assume safely that the factor $2 \lambda_{1}^{2}+\lambda_{2}^{2}+\lambda_{3}^{2}+2 \lambda_{1} \lambda_{2}$ without $\lambda_{\phi h}$ effectively determines the stability of Higgs vacuum in relic density direct search and indirect search allowed parameter space. It turns out that when $\lambda_{H}>0$. all other copositivity conditions for all relic and DD cross section satisfied points in our model stays positive from $\mu=m_{t}$ to $\mu=M_{P}$.

In figure 25, we constrain relic density direct search and indirect search allowed points of the model in $2 \lambda_{1}^{2}+\lambda_{2}^{2}+\lambda_{3}^{2}+2 \lambda_{1} \lambda_{2}-\lambda_{1}$ plane to additionally satisfy perturbativity and vacuum stability conditions following eqs. (3.1)-(3.5). Orange points satisfy relic density, DD bounds and indirect search bound, while the green, blue and red points on top of that satisfy perturbativity and vacuum stability conditions upto high energy scales $\mu=10^{10} \mathrm{GeV}, 10^{16} \mathrm{GeV}$ and $10^{19} \mathrm{GeV}$ respectively. This very figure essentially shows that all those points with either small values of $\lambda_{1}$ (i.e. small $m_{H^{ \pm}}-m_{H^{0}}$ )are discarded due to stability of EW vacuum, while those with large $\lambda_{1}$ (i.e. large $m_{H^{ \pm}}-m_{H^{0}}$ )are discarded by perturbative limits of the coupling at high scale.

In figure 26 we study the correlation between the individual scalar couplings to satisfy the DM constraints, perturbativity limits and vacuum stability criteria. In left panel of figure 26, we show the DM relic density and DD cross section satisfied points in $\lambda_{2}-\lambda_{3}$ 

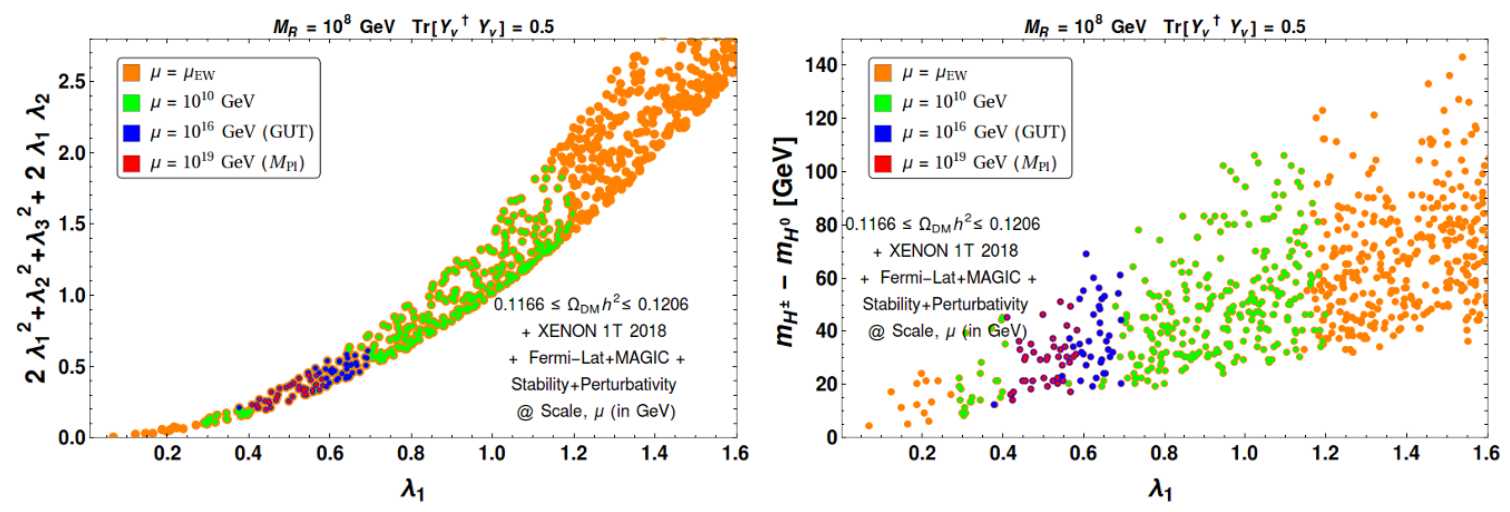

Figure 25. Relic density, direct search and indirect search allowed points in $2 \lambda_{1}^{2}+\lambda_{2}^{2}+\lambda_{3}^{2}+2 \lambda_{1} \lambda_{2}$ versus $\lambda_{1}$ plane (left figure) and $m_{H^{ \pm}}-m_{H^{0}}$ versus $\lambda_{1}$ plane (right figure) at $\mathrm{EW}$ scale (orange points). We also find high scale validity of the model following eqs. (3.1), (3.4) and (3.5) by considering the high scale to be $10^{10} \mathrm{GeV}$ (green), $10^{16} \mathrm{GeV}$ (blue) and $10^{19} \mathrm{GeV}$ (red). Right handed neutrino mass and Yukawa couplings are kept fixed at $M_{R}=10^{8} \mathrm{GeV}, \operatorname{Tr}\left[Y_{\nu}^{\dagger} Y_{\nu}\right]=0.5$ with $m_{t}=173.1 \mathrm{GeV}$.
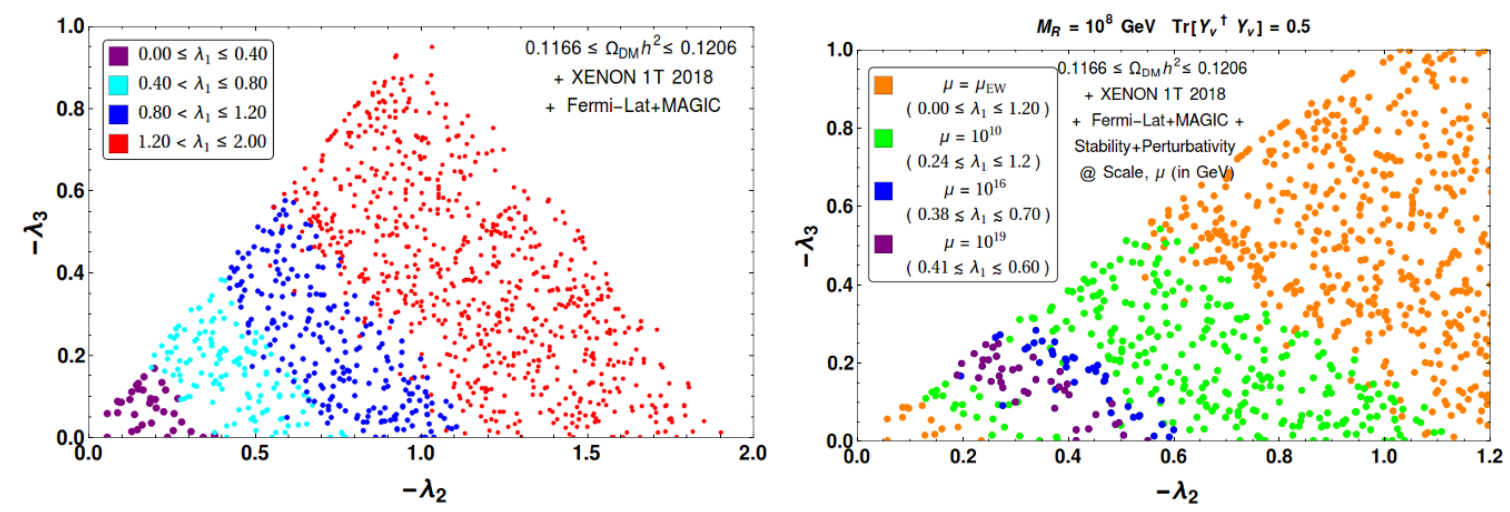

Figure 26. Relic density direct search and indirect search allowed points in $\lambda_{2}-\lambda_{3}$ plane for different values of $\lambda_{1}$ (left). Allowed points in the same parameter space from DM constraints (orange), stability and perturbativity conditions following eqs. (3.1)-(3.5) considering the high scale $\mu=\mu_{\mathrm{EW}}, 10^{10} \mathrm{GeV}$ (green), $10^{16} \mathrm{GeV}$ (blue) and $10^{19} \mathrm{GeV}$ (purple) (right).

plane for different values of $\lambda_{1}$. In right panel we first identify the relevant parameter space in the same plane which satisfy the DM constraints, the perturbativity bound and vacuum stability criteria till EW energy scale. Then we further impose perturbativity bound and vacuum stability conditions considering the high scale as $\mu=10^{10} \mathrm{GeV}, 10^{16} \mathrm{GeV}$ and $10^{19} \mathrm{GeV}$ in addition to the DM constraints. It is seen that the lower portion of the available parameter space gets discarded by vacuum stability or high value of $\lambda_{c}$ while perturbativity bounds constrain the higher values of the couplings. In this plot also we kept $M_{R}=10^{8} \mathrm{GeV}, \operatorname{Tr}\left[Y_{\nu}^{\dagger} Y_{\nu}\right]=0.5$ with $m_{t}=173.1 \mathrm{GeV}$. We must also note that with larger $M_{R}$ and smaller Yukawa $\operatorname{Tr}\left[Y_{\nu}^{\dagger} Y_{\nu}\right]$, we could obtain a larger available parameter space from high scale validity.

In figure 27, we show the high scale validity of relic density and direct search allowed parameter space of the model in $m_{H^{0}}-m_{A^{0}}$ (top left), $m_{H^{0}}-m_{H^{ \pm}}$(top right) and 

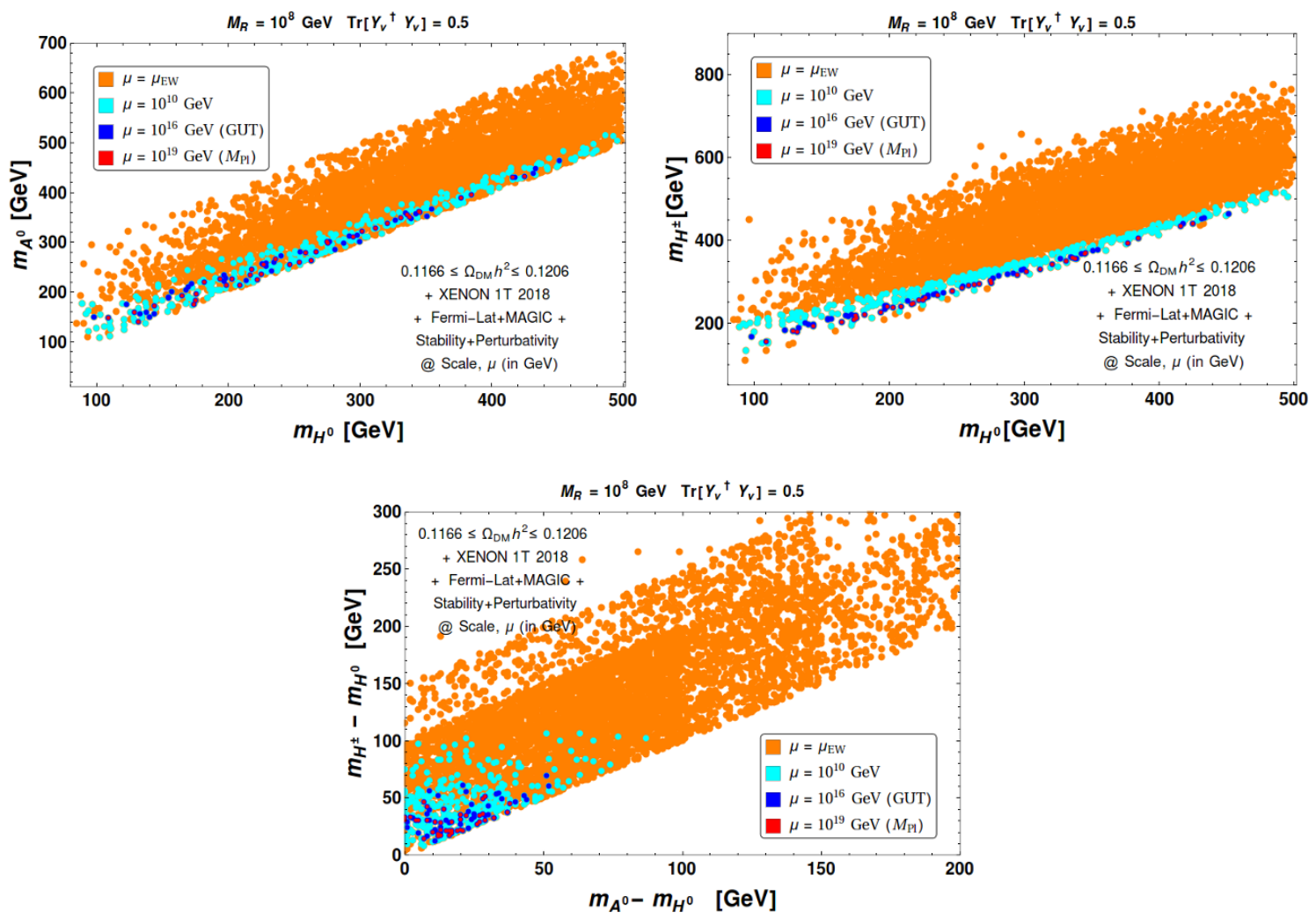

Figure 27. Relic density direct search and indirect search allowed points (orange) in $m_{A^{0}}-m_{H^{0}}$ (top left), $m_{H^{ \pm}}-m_{H^{0}}$ (top left) and $\Delta m\left(=m_{A^{0}}-m_{H^{0}}\right)-\Delta M\left(=m_{H^{ \pm}}-m_{H^{0}}\right)$ (bottom). We further apply stability and perturbativity conditions following eqs. (3.1)-(3.5) at different energy scales $\mu=10^{10} \mathrm{GeV}$ (light blue), $10^{16} \mathrm{GeV}$ (dark blue) and $10^{19} \mathrm{GeV}$ (red).

$\Delta m\left(=m_{A^{0}}-m_{H^{0}}\right)-\Delta M\left(=m_{H^{ \pm}}-m_{H^{0}}\right)$ planes with $M_{R}=10^{8} \mathrm{GeV}$ and $\operatorname{Tr}\left[Y_{\nu}^{\dagger} Y_{\nu}\right]=0.5$ at different high energy scales $\mu=\left\{10^{10}, 10^{16}, 10^{19}\right\}$ GeVs denoted by light blue, dark blue and red points. The orange points are corresponding to relic and direct search allowed parameter space at EW scale. We see that larger mass difference between inert higgs components, $\Delta m-\Delta M$ which are related with quartic couplings, $\lambda_{1,2,3}$ (see eq. (2.7)), are discarded from perturbativity conditions mentioned in eq. (3.4). While the small mass differences between inert componets are also excluded from stability criteria of Higgs potential.

Till now, while discussing the effect of stability and high scale validity of the proposed set up, we have considered fixed right handed neutrino mass, $M_{R}=10^{8} \mathrm{GeV}$ and corresponding Yukawa coupling $\operatorname{Tr}\left[Y_{\nu}^{\dagger} Y_{\nu}\right]=0.5$. For sake of completeness we extend our study for few different values of RH neutrino mass and $\operatorname{Tr}\left[Y_{\nu}^{\dagger} Y_{\nu}\right]$ and find out the allowed ranges of the relevant parameters considering both the hierarchies $m_{\phi}>m_{H^{0}}$ and $m_{\phi} \leq m_{H^{0}}$ separately. We note the corresponding results in table 9 and table 10 of appendix C.

\section{Collider signature of inert doublet DM at LHC}

Inert doublet has been an attractive DM framework, due to the possibility of collider detection $[52,53]$. Here, we relook into the possible collider search strategies of IDM at 

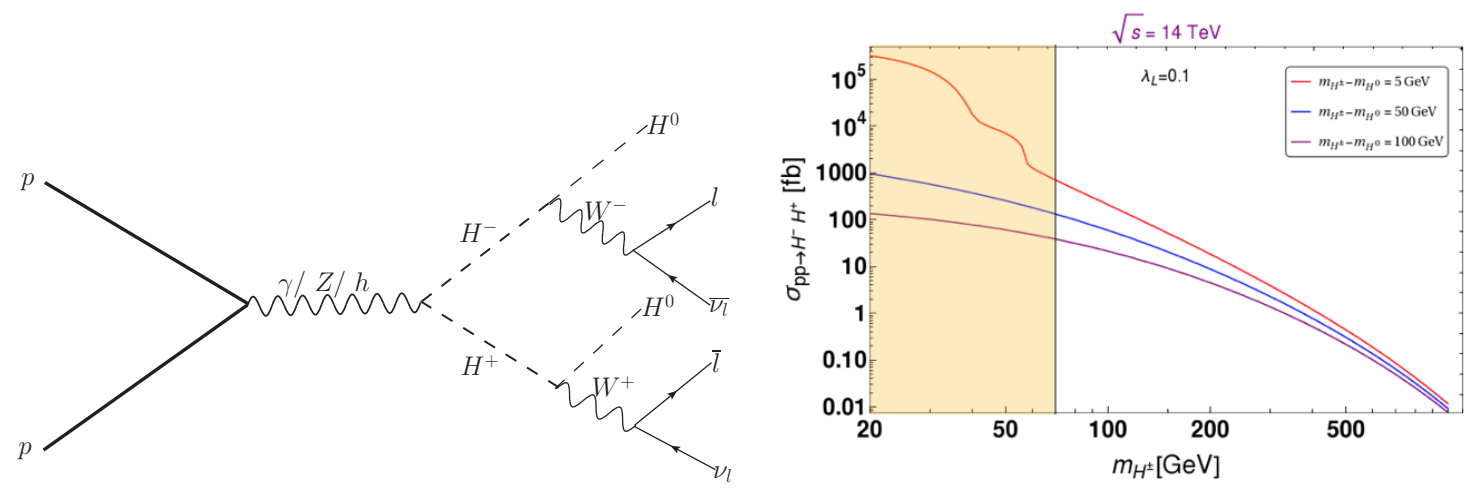

Figure 28. [Left] Feynman graph for OSDL $+\mathbb{E}_{T}$ signature of IDM at LHC. [Right] Variation of production cross-section $\sigma_{p p \rightarrow H^{+} H^{-}}$(in fb) with $m_{H^{ \pm}}\left(=m_{H^{0}}+\Delta M\right)$ in $\mathrm{GeV}$ for different choices of $m_{H^{ \pm}}-m_{H^{0}}$ for center-of-mass energy $\sqrt{s}=14 \mathrm{TeV}$ at LHC. LEP limit on charged scalar is shown by the shaded region.

LHC in presence of a second scalar singlet DM component. It is also worth mentioning here that the real scalar singlet, which interacts with SM only through Higgs portal coupling, does not have any promising collider signature excepting mono- $X$ signature arising out of initial state radiation (ISR), where $X$ stands for $W, Z$, jet. Such signals are heavily submerged in SM background due to weak production cross-section of DM in relic density and direct search allowed parameter space [51]. The charge components $H^{+}, H^{-}$of inert DM can be produced at LHC via Drell-Yan $Z$ and $\gamma$ mediation as well as through Higgs mediation. Further decay of $H^{ \pm}$to DM $\left(H^{0}\right)$ and leptonic final states through on/off shell $W^{ \pm}$yields hadronically quiet opposite sign di-lepton plus missing energy $\left(\mathrm{OSDL}+\not_{T}\right){ }^{4}$ as shown in left panel of figure 28. In this study, we focus on this particular signal of inert dark matter as detailed below:

$$
\begin{aligned}
\text { Signal :: OSDL }+\mathbb{E}_{T} \equiv \ell^{+} \ell^{-}+\left(\mathbb{E}_{T}\right): \quad & p p \rightarrow H^{+} H^{-},\left(H^{-} \rightarrow \ell^{-} \overline{\nu_{\ell}} H^{0}\right), \\
& \left(H^{+} \rightarrow \ell^{+} \nu_{\ell} H^{0}\right) \text {; where } \ell=\{e, \mu\} .
\end{aligned}
$$

In the right panel of figure 28, we show variation of charged pair $\left(H^{+} H^{-}\right)$production crosssection at LHC for center-of-mass energy $\sqrt{s}=14 \mathrm{TeV}$ as a function of $m_{H^{ \pm}}=m_{H^{0}}+\Delta M$, where $\Delta M$ indicates the mass difference with the inert DM and serves as a very important variable for the signal characteristics. The plot on r.h.s. show that production crosssection is decreasing with larger charged scalar mass $m_{H^{ \pm}}$, where we have demonstrated three fixed values of $m_{H^{ \pm}}-m_{H^{0}}=5,50$ and $100 \mathrm{GeV}$. Around $m_{H^{ \pm}} \sim m_{h} / 2$, there is a sharpe fall of production cross-section. This is because, for $m_{H^{ \pm}} \leq m_{h} / 2$, there is a significant contribution arising from Higgs production and its subsequent decay to the charged scalar components, which otherwise turns into an off-shell propagator to yield a subdued contribution to Drell-Yan production. Following [116], a conservative bound on the charge scalars is applied here as $m_{H^{ \pm}} \geq 70 \mathrm{GeV}$, as indicated in the r.h.s. plot of figure 28.

\footnotetext{
${ }^{4}$ There are other possible signatures (for example, three lepton final state) of inert DM arising from the combination of $H^{ \pm}, A^{0}$ production and their subsequent decays, for a detailed list see $[44,53]$.
} 


\begin{tabular}{|c|c|c|c|c|c|}
\hline BPs & $\left\{m_{H^{0}}, m_{\phi}, m_{A^{0}}, m_{H^{ \pm}}, \lambda_{L}, \lambda_{\phi h}, \lambda_{c}\right\}$ & $\Omega_{H^{0}} h^{2}$ & $\Omega_{\phi} h^{2}$ & $\sigma_{H^{0}}^{\text {eff }}\left(\mathrm{cm}^{2}\right)$ & $\sigma_{\phi}^{\text {eff }}\left(\mathrm{cm}^{2}\right)$ \\
\hline BPC1 & $\{209,483,213,239,0.006,0.09,0.13\}$ & 0.0082 & 0.1084 & $3.7 \times 10^{-47}$ & $2.8 \times 10^{-46}$ \\
\hline BPC2 & $\{129,476,158,180,0.011,0.069,0.13\}$ & 0.0032 & 0.1175 & $6.7 \times 10^{-47}$ & $1.8 \times 10^{-46}$ \\
\hline BPC3 & $\{100,96,127,178,0.010,0.002,0.56\}$ & 0.0021 & 0.1156 & $6.8 \times 10^{-47}$ & $3.7 \times 10^{-48}$ \\
\hline
\end{tabular}

\begin{tabular}{|c|c|c|c|c|}
\hline BPs & $m_{H^{ \pm}}-m_{H^{0}}$ & $\left\{\lambda_{1}, \lambda_{2}, \lambda_{3}\right\}$ & $\left\{M_{R}, \operatorname{Tr}\left[Y_{\nu}^{\dagger} Y_{\nu}\right]\right\}$ & Validity Scale $(\mu)$ \\
\hline BPC1 & $30\left(<m_{W}\right)$ & $\{0.456,-0.416,-0.028\}$ & $\left\{10^{8}, 0.5\right\}$ & $1.22 \times 10^{19}\left(M_{p l}\right)$ \\
\hline BPC2 & $51\left(<m_{W}\right)$ & $\{0.543,-0.383,-0.137\}$ & $\left\{10^{8}, 0.5\right\}$ & $1.22 \times 10^{19}\left(M_{p l}\right)$ \\
\hline BPC3 & $78\left(\sim m_{W}\right)$ & $\{0.737,-0.615,-0.101\}$ & $\left\{10^{8}, 0.5\right\}$ & $\sim 10^{14}$ \\
\hline
\end{tabular}

Table 4. DM masses, quartic couplings, relic densities and spin independent effective DM-neucleon cross-section of selected benchmark points for collider study. All benchmark points chosen here have $m_{H^{ \pm}}-m_{H^{0}}<m_{W^{ \pm}}$for off-shell production of $W^{ \pm}$. The maximum scale $(\mu)$ of Higgs vacuum satability and peturbativity in presence of right handed neutrinos are also noted. All masses and scales are in $\mathrm{GeV}$.

\begin{tabular}{|c|c|c|c|c|c|}
\hline BPs & $\left\{m_{H^{0}}, m_{\phi}, m_{A^{0}}, m_{H^{ \pm}}, \lambda_{L}, \lambda_{\phi h}, \lambda_{c}\right\}$ & $\Omega_{H^{0}} h^{2}$ & $\Omega_{\phi} h^{2}$ & $\sigma_{H^{0}}^{\text {eff }}\left(\mathrm{cm}^{2}\right)$ & $\sigma_{\phi}^{\text {eff }}\left(\mathrm{cm}^{2}\right)$ \\
\hline BPD1 & $\{92,386,155,198,0.004,0.063,0.10\}$ & 0.0032 & 0.1167 & $4.5 \times 10^{-47}$ & $2.2 \times 10^{-46}$ \\
\hline BPD2 & $\{90,437,136,233,0.006,0.077,0.11\}$ & 0.0030 & 0.1150 & $6.9 \times 10^{-47}$ & $2.6 \times 10^{-46}$ \\
\hline
\end{tabular}

\begin{tabular}{|c|c|c|c|c|}
\hline BPs & $m_{H^{ \pm}}-m_{H^{0}}$ & $\left\{\lambda_{1}, \lambda_{2}, \lambda_{3}\right\}$ & $\left\{M_{R}, \operatorname{Tr}\left[Y_{\nu}^{\dagger} Y_{\nu}\right]\right\}$ & Validity Scale $(\mu)$ \\
\hline BPD1 & $106\left(>m_{W}\right)$ & $\{1.024,-0.7588,-0.2571\}$ & $\left\{10^{8}, 0.5\right\}$ & $10^{10}$ \\
\hline BPD2 & $143\left(>m_{W}\right)$ & $\{1.538,-1.354,-0.1718\}$ & $\left\{10^{6}, 0.5\right\}$ & $10^{8}$ \\
\hline
\end{tabular}

Table 5. DM masses, quartic couplings, relic densities and spin independent effective DM-neucleon cross-section of selected benchmark points for collider study. All benchmark points chosen here have $m_{H^{ \pm}}-m_{H^{0}}>m_{W^{ \pm}}$for on-shell production of $W^{ \pm}$. The maximum scale $(\mu)$ of Higgs vacuum satability and peturbativity in presence of right handed neutrinos are also noted. All masses and scales are in $\mathrm{GeV}$.

We next choose a set of benchmark points (BPs) allowed from DM relic, direct search constraints as well as from Higgs invisible decay constraints for performing collider simulation, shown in table 4 and table 5. The BPs are also allowed from absolute Higgs vacuum stability and perturbativity limits in presence of right handed neutrinos, valid upto scale $\mu$ as mentioned in the tables. The benchmark points are divided into two categories: (BPC1BPC3) in table 4 correspond to $\Delta M=m_{H^{ \pm}}-m_{H^{0}} \lesssim m_{W^{ \pm}}$where the charged scalar, $H^{ \pm}$decay through off-shell $W^{ \pm}$. On the other hand, benchmark points (BPD1-BPD2) in table 5 correspond to $\Delta M=m_{H^{ \pm}}-m_{H^{0}}>m_{W^{ \pm}}$, where the charged scalar $H^{ \pm}$decay through on-shell $W^{ \pm}$. Each table (table 4 and table 5) consists of two parts: the first part contains all the relevant dark sector masses, couplings, relic density and direct search cross-sections of both DM components. The second part demonstrates the mass difference $\left(\Delta M=m_{H^{ \pm}}-m_{H^{0}}\right)$, choice of right handed neutrino mass, neutrino Yukawa and the maximum scale of validity $(\mu)$ of the Higgs vacuum. 
The simulation technique adopted here is as follows. We first implemented the model in FeynRule [117] to generate UFO file which is required to feed into event generator Madgraph[118]. Then these events are passed to Pythia [119] for hadronization. All parton level leading order (LO) signal events and SM background events ${ }^{5}$ are generated in Madgraph at $\sqrt{s}=14 \mathrm{TeV}$ using cteq611 [120] parton distribution. Leptons $(\ell=e, \mu)$ isolation, jet and unclustered event formation to mimic to the actual collider environment are performed as follows:

(i) Lepton isolation. The minimum transverse momentum required to identify a lepton $(\ell=e, \mu)$ has been kept as $p_{T}>20 \mathrm{GeV}$ and we also require the lepton to be produced in the central region of detector followed by pseudorapidity selection as $|\eta|<2.5$. Two leptons are separated from each other with minimum distance $\Delta R \geq 0.2$ in $\eta-\phi$ plane. To separate leptons from jets we further imposed $\Delta R \geq 0.4$.

(ii) Jet formation. For jet formation, we used cone algorithm PYCELL in built in Pythia. All partons within a cone of $\Delta R \leq 0.4$ around a jet initiator with $p_{T}>20 \mathrm{GeV}$ is identified to form a jet. It is important to identify jets in our case because we require the final state signal to be hadronically quiet i.e. to have zero jets.

(iii) Unclustered Objects. All final state objects with $0.5<p_{T}<20 \mathrm{GeV}$ and $2.5<|\eta|<5$ are considered as unclustered objects. Those objects neither form jets nor identified as isolated leptons and they only contribute to missing energy.

The main idea is to see if the signal events rise over SM background. For that there are three key kinematic variables where the signal and background show different sensitivity. They are:

- Missing Energy $\left(E_{T}\right)$. The most important signature of DM being produced at collider. This is defined by a vector sum of transverse momentum of all the missing particles (those are not registered in the detector); this in turn can be estimated form the momentum imbalance in the transverse direction associated to the visible particles. Thus missing energy (MET) is defined as:

$$
E_{T}=-\sqrt{\left(\sum_{\ell, j} p_{x}\right)^{2}+\left(\sum_{\ell, j} p_{y}\right)^{2}}
$$

where the sum runs over all visible objects that include the leptons, jets and the unclustered components.

- Transverse Mass $\left(H_{T}\right)$. Transverse mass of an event is identified with the scalar sum of the transverse momentum of objects reconstructed in a collider event, namely lepton and jets as defined above.

$$
H_{T}=\sum_{\ell, j} \sqrt{\left(p_{x}\right)^{2}+\left(p_{y}\right)^{2}}
$$

\footnotetext{
${ }^{5}$ There are several SM process which contribute to the chosen $\ell^{+} \ell^{-}+\left(\mathbb{E}_{T}\right)$ signal, dominant processes are: $t \bar{t}, W^{+} W^{-}, Z Z$ and $W^{+} W^{-} Z$.
} 

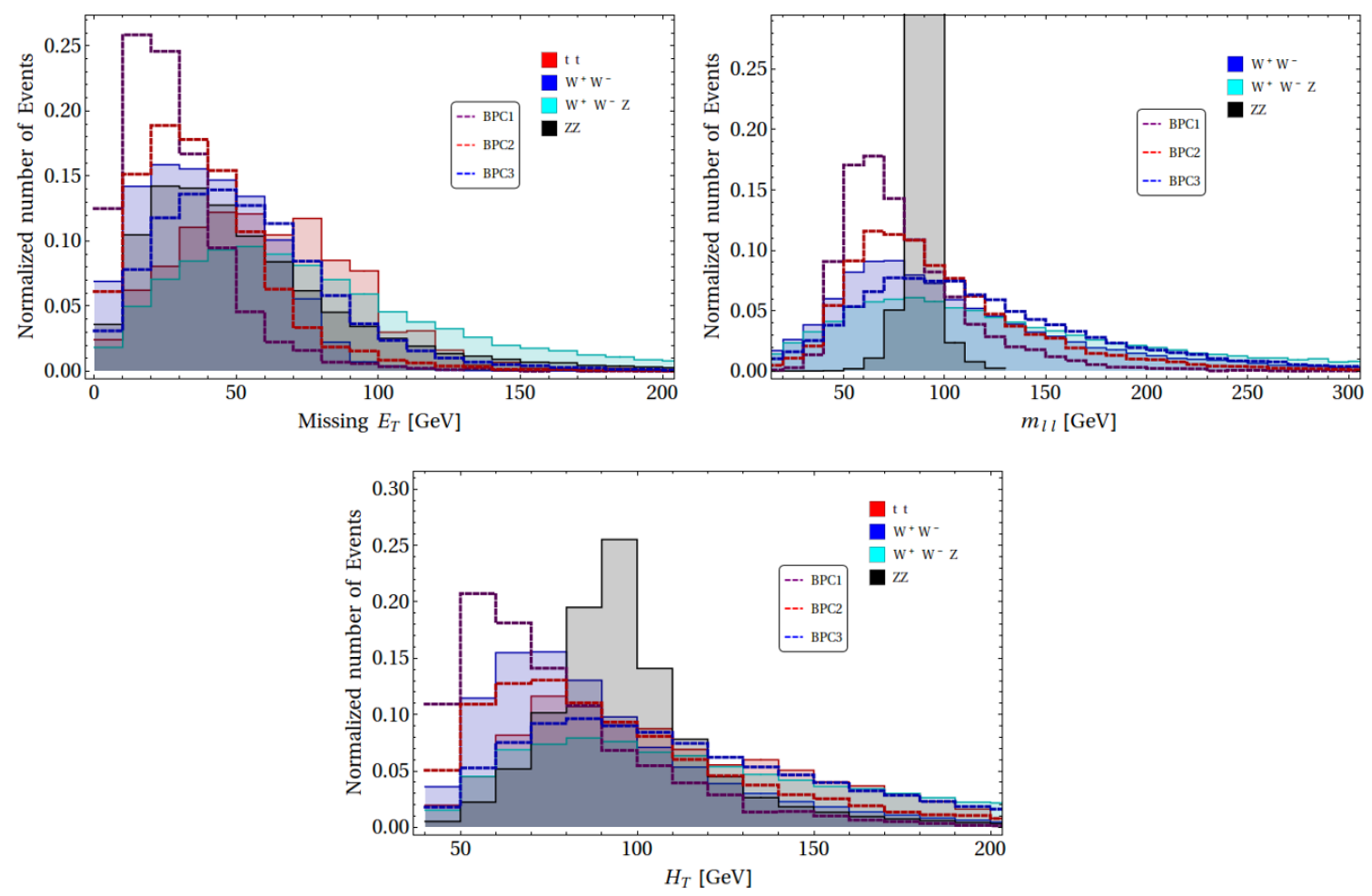

Figure 29. Distribution of missing energy $\left(\mathbb{E}_{T}\right)$, invariant mass of OSDL $\left(m_{\ell^{+} \ell^{-}}\right)$and transverse mass $\left(H_{T}\right)$ for signal events $\ell^{+} \ell^{-}+\left(\mathbb{E}_{T}\right)$ and dominant SM background events at LHC with $\sqrt{s}=14 \mathrm{TeV}$.

- Invariant mass $\left(m_{\ell \ell}\right)$. Invariant mass of opposite sign dilepton hints to the parent particle, from which the leptons have been produced and thus helps segregating signal from background. This is defined as:

$$
m_{\ell^{+} \ell^{-}}=\sqrt{\left(\sum_{\ell^{+} \ell^{-}} p_{x}\right)^{2}+\left(\sum_{\ell^{+} \ell^{-}} p_{y}\right)^{2}+\left(\sum_{\ell^{+} \ell^{-}} p_{z}\right)^{2}} .
$$

The distribution of missing energy $\left(\mathbb{E}_{T}\right)$, invariant mass of opposite sign dilepton $\left(m_{\ell^{+} \ell^{-}}\right)$ and transverse mass $\left(H_{T}\right)$ for the BPs along with dominant SM background events are shown in figure 29 top left, top right and bottom panel respectively. All BPs depicted in figure 29 correspond to $m_{H^{ \pm}}-m_{H^{0}} \equiv \Delta M<m_{W^{ \pm}}$where opposite sign di-lepton are produced from off-shell $W^{ \pm}$mediator. All the distributions are normalised to one event. Missing energy (as well as $H_{T}$ ) distributions of BPs (BPC1-BPC3) show that the peak of distribution for the signal is on the left of SM background. This is because the benchmark points are characterised by small $\Delta M$, where the charged scalars and inert DM have small mass splitting. Therefore, such situations are visibly segregated from SM background by MET and $H_{T}$ distribution. Clearly, when $\Delta M$ becomes $m_{W^{ \pm}}$(for example, BPC3) the distribution closely mimic SM background. Therefore the signal events for this class of benchmark points can survive for a suitable upper $\mathbb{E}_{T}$ and $H_{T}$ cut while reducing SM backgrounds. It is important to take a note that OSDL events coming from $Z Z$ background 

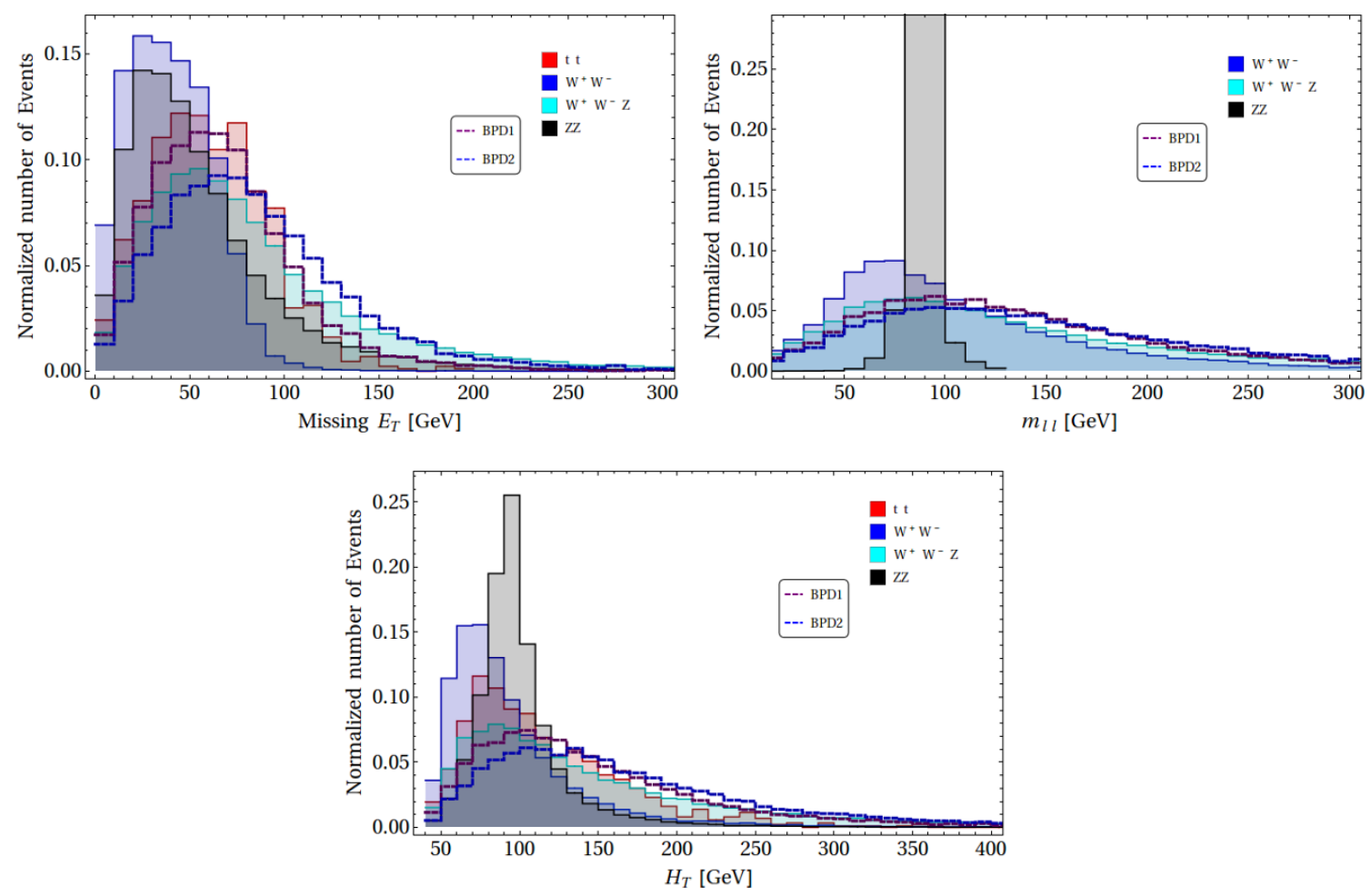

Figure 30. Distribution of missing energy $\left(\mathbb{E}_{T}\right)$, invariant mass of OSDL $\left(m_{\ell^{+} \ell^{-}}\right)$and transverse mass $\left(H_{T}\right)$ for signal events $\ell^{+} \ell^{-}+\left(\mathbb{E}_{T}\right)$ and dominant SM background events at LHC with $\sqrt{s}=14 \mathrm{TeV}$.

naturally peaks at $m_{Z}$ in $m_{\ell \ell}$ distribution. Therefore, we use invariant mass cut in the $Z$ mass window to get rid of this background.

The situation is reversed for larger splitting between the charged scalar component with DM, i.e $\Delta M \equiv m_{H^{ \pm}}-m_{H^{0}}>m_{W^{ \pm}}$corresponding to BPs (BPD1-BPD2) as in table 5. The distributions of $\mathscr{E}_{T}, m_{\ell \ell}$ and $H_{T}$ therefore become flatter and peak of the distribution shifts to higher value as shown in figure 30. In such cases the signal events for large $\Delta M$ can be separated from SM background at a suitable lower end cut of $\mathbb{E}_{T}$ and $H_{T}$.

Therefore the selection cuts used in this analysis are summarised as follows:

- Invariant mass $\left(m_{\ell \ell}\right)$ cuts: $m_{\ell \ell}<\left(m_{Z}-15\right) \mathrm{GeV}$ and $m_{\ell \ell}>\left(m_{Z}+15\right) \mathrm{GeV}$.

- $H_{T}$ cuts:

- $H_{T}<70$ when $m_{H^{ \pm}}-m_{H^{0}}<m_{W^{ \pm}}$.

- $H_{T}>150,200$ when $m_{H^{ \pm}}-m_{H^{0}}>m_{W^{ \pm}}$.

- $\mathbb{E}_{T}$ cuts:

- $\mathbb{E}_{T}<30,40$ when $m_{H^{ \pm}}-m_{H^{0}}<m_{W^{ \pm}}$.

- $\mathbb{E}_{T}>100,150$ when $m_{H^{ \pm}}-m_{H^{0}}>m_{W^{ \pm}}$. 


\begin{tabular}{|c|c|c|c|c|c|}
\hline BPs & $\sigma^{\mathrm{OSD}}(\mathrm{fb})$ & $\mathscr{E}_{T}(\mathrm{GeV})$ & $H_{T}(\mathrm{GeV})$ & $\sigma_{\text {eff }}^{\text {OSD }}(\mathrm{fb})$ & $N_{\text {eff }}^{\mathrm{OSD}} \mathcal{L}=10^{2} \mathrm{fb}^{-1}$ \\
\hline BPC1 & 9.16 & $<30$ & $<70$ & 0.28 & 28 \\
& & $<40$ & & 0.33 & 33 \\
\hline BPC2 & 27.14 & $<30$ & $<70$ & 0.72 & 72 \\
& & $<40$ & & 0.97 & 97 \\
\hline BPC3 & 28.65 & $<30$ & $<70$ & 0.35 & 35 \\
& & $<40$ & & 0.55 & 55 \\
\hline
\end{tabular}

Table 6. Signal cross-section for BPC1-BPC3 after the selection cuts are employed.

\begin{tabular}{|c|c|c|c|c|c|}
\hline BPs & $\sigma^{\mathrm{OSD}}(\mathrm{fb})$ & $\mathbb{E}_{T}(\mathrm{GeV})$ & $H_{T}(\mathrm{GeV})$ & $\sigma_{\text {eff }}^{\mathrm{OSD}}(\mathrm{fb})$ & $N_{\mathrm{eff}}^{\mathrm{OSD}} @ \mathcal{L}=10^{2} \mathrm{fb}^{-1}$ \\
\hline BPD1 & \multirow{2}{*}{19.91} & $>100$ & $>150$ & 0.75 & 75 \\
& & & $>200$ & 0.48 & 48 \\
\cline { 3 - 6 } & & $>150$ & $>150$ & 0.23 & 23 \\
& & & $>200$ & 0.21 & 21 \\
\hline BPD2 & \multirow{2}{*}{11.16} & $>100$ & $>150$ & 0.87 & 87 \\
& & & $>200$ & 0.59 & 59 \\
\cline { 3 - 6 } & & $>150$ & $>150$ & 0.34 & 34 \\
& & & $>200$ & 0.29 & 29 \\
\hline
\end{tabular}

Table 7. Signal cross-section for BPD1-BPD2 after the selection cuts are employed.

We next turn to signal and background events that survive after the selection cuts are employed. The signal events are listed in table 6 for BPC1-BPC3.

Similarly, signal events for BPD1-BPD2 are listed in table 7 using a lower cut on $\mathbb{E}_{T}$ and $H_{T}$. The signal cross-section and event numbers for this class of points are much smaller due to the fact that the charged scalar masses are on higher side as for all of the cases $\Delta m>m_{W}$ independent of DM masses. However, the SM background events get even more suppressed with the set of $\mathbb{E}_{T}, H_{T}$ cuts. The SM background cross-section and event numbers after cut flow is mentioned in table 8. Therefore, in spite of smaller signal events in this region of parameter space with BPD benchmark points, the discovery potential of the signal requires similar luminosity to that of BPC cases.

Finally we present the discovery reach of the signal events in terms of significance $\sigma=\frac{S}{\sqrt{S+B}}$, where $S$ denotes signal events and $B$ denotes SM background events in terms of luminosity. This is shown in figure 31 . This shows that the benchmark points that characterise the two component DM framework, can yield a visible signature at high luminosity with $\mathcal{L} \sim 500 \mathrm{fb}^{-1}$ depending on the charged scalar mass and its splitting with DM for the case $m_{H^{ \pm}}-m_{H^{0}}>m_{W^{ \pm}}$.

\section{Summary and conclusions}

We have studied a two component scalar DM model in presence of right handed neutrinos that address neutrino mass generation through type I seesaw. The DM components are 


\begin{tabular}{|c|c|c|c|c|c|}
\hline SM Bkg. & $\sigma^{\mathrm{OSD}}(\mathrm{fb})$ & $\mathbb{E}_{T}(\mathrm{GeV})$ & $H_{T}(\mathrm{GeV})$ & $\sigma_{\mathrm{eff}}^{\mathrm{OSD}}(\mathrm{fb})$ & $N_{\mathrm{eff}}^{\mathrm{OSD}} @ \mathcal{L}=10^{2} \mathrm{fb}^{-1}$ \\
\hline \multirow{6}{*}{$t \bar{t}$} & \multirow{6}{*}{$36.69 \times 10^{3}$} & $<30$ & $<70$ & 6.23 & 623 \\
\hline & & $<40$ & & 10.27 & 1027 \\
\hline & & $>100$ & $>150$ & 10.64 & 1064 \\
\hline & & & $>200$ & 4.40 & 440 \\
\hline & & $>150$ & $>150$ & 1.47 & 147 \\
\hline & & & $>200$ & 1.10 & 110 \\
\hline \multirow{6}{*}{$W^{+} W^{-}$} & \multirow{6}{*}{$4.74 \times 10^{3}$} & $<30$ & $<70$ & 131.18 & 13118 \\
\hline & & $<40$ & & 187.89 & 18789 \\
\hline & & $>100$ & $>150$ & 7.72 & 772 \\
\hline & & & $>200$ & 4.97 & 4.97 \\
\hline & & $>150$ & $>150$ & 1.23 & 123 \\
\hline & & & $>200$ & 1.18 & 118 \\
\hline \multirow{6}{*}{$Z Z$} & \multirow{6}{*}{$0.25 \times 10^{3}$} & $<30$ & $<70$ & 0.53 & 53 \\
\hline & & $<40$ & & 0.69 & 69 \\
\hline & & $>100$ & $>150$ & 0.18 & 18 \\
\hline & & & $>200$ & 0.05 & 5 \\
\hline & & $>150$ & $>150$ & 0.09 & 9 \\
\hline & & & $>200$ & 0.04 & 4 \\
\hline \multirow{6}{*}{$W^{+} W^{-} Z$} & \multirow{6}{*}{1.00} & $<30$ & $<70$ & 0.01 & 1 \\
\hline & & $<40$ & & 0.02 & 2 \\
\hline & & $>100$ & $>150$ & 0.06 & 6 \\
\hline & & & $>200$ & 0.04 & 4 \\
\hline & & $>150$ & $>150$ & 0.03 & 3 \\
\hline & & & $>200$ & 0.02 & 2 \\
\hline
\end{tabular}

Table 8. Dominant SM background contribution to $\ell^{+} \ell^{-}+\left(\mathbb{E}_{T}\right)$ signal events for $\sqrt{s}=14 \mathrm{TeV}$ at LHC. The effective number of final state background events with different $\mathbb{E}_{T}, H_{T}$ and $m_{\ell \ell}$ cuts are tabulated for luminosity $\mathcal{L}=100 \mathrm{fb}^{-1}$. To incorporate the Next-to-Leading order (NLO) cross section of SM background we have used appropriate K-factors [121].

(i) a singlet scalar and (ii) an inert scalar doublet, both studied extensively as single component DM in literature. We show that the presence of second component enlarges the available parameter space significantly considering relic density, direct search and indirect search constraints. In particular, the inert scalar DM will now be allowed in the so called 'desert region': $\left\{m_{W}-550\right\} \mathrm{GeV}$. Also for singlet scalar, we can now revive it below $\mathrm{TeV}$, which is otherwise discarded (except Higgs resonance) from direct search in single component framework. The results obtained for DM analysis crucially depends on DM-DM conversion, which have been demonstrated in details.

We also study the high scale perturbativity and vacuum stability of the Higgs potential by analysing two loop RGE $\beta$ functions. This in turn puts further constraints on the available DM parameter space of the model. One of the important conclusions obtained are that the mass splitting of the charged scalar component to the corresponding DM 

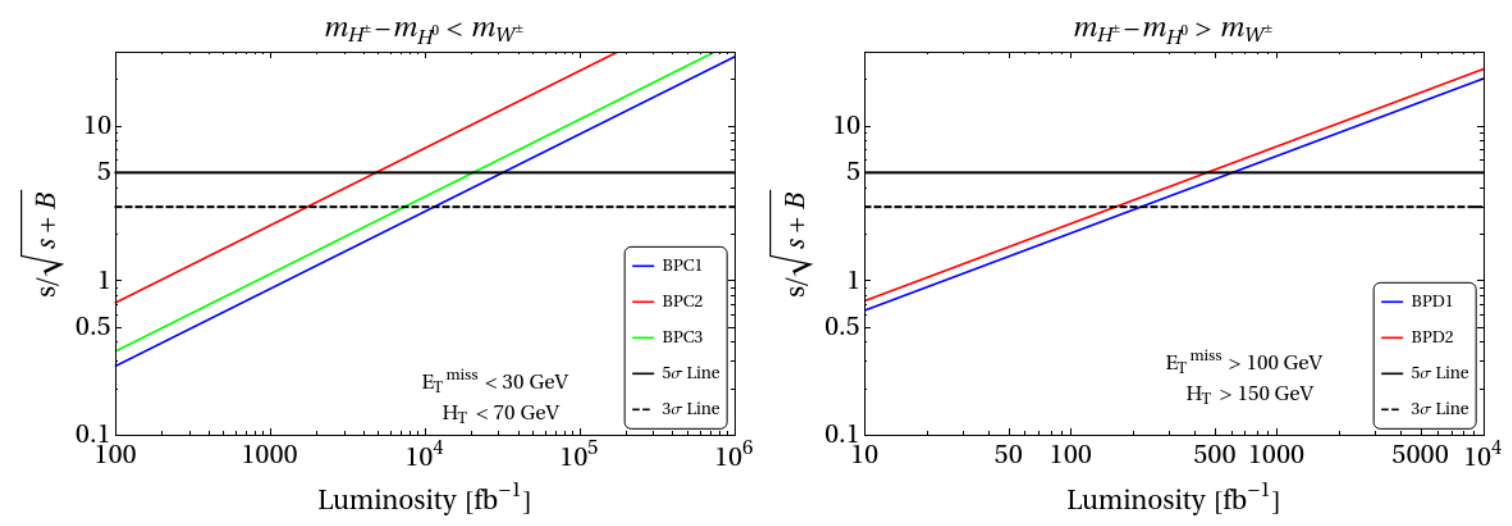

Figure 31. Signal significance of some select benchmark points at LHC for $\sqrt{s}=14 \mathrm{TeV}$, in terms of Luminosity $\left(\mathrm{fb}^{-1}\right) .3 \sigma$ and $5 \sigma$ lines are shown. Left: Points with $\Delta M<m_{W}$; Right: Points with $\Delta M>m_{W}$.

component of inert doublet is crucially tamed depending on the absolute stability scale of the scalar potential, coming from the perturbativity constraint on the quartic and Yukawa coupling. For example, we find:

- Validity scale $(\mu) \sim$ intermediate scale $\left(10^{10} \mathrm{GeV}\right): \Delta M=m_{H^{ \pm}}-m_{H^{0}} \sim\{8-$ $106\} \mathrm{GeV}$,

- Validity scale $(\mu) \sim$ Planck scale $\left(10^{19} \mathrm{GeV}\right): \Delta M=m_{H^{ \pm}}-m_{H^{0}} \sim\{14-51\} \mathrm{GeV}$,

with RH neutrino mass $M_{R}=10^{8} \mathrm{GeV}$ and Yukawa $\operatorname{Tr}\left[Y_{\nu}^{\dagger} Y_{\nu}\right]=0.5$. The presence of RHNs in the model not only helps us addressing the neutrino masses but also controls the high scale validity of the model parameters, for example, low $\Delta M$ regions. This is how the neutrino and dark sector constraints affect each other.

Inert Higgs having charged scalars have collider detectability. We point out that the collider search prospect of the charged components are not only limited to low DM masses $\left(<m_{W}\right)$, but is open to a larger mass range in presence of the second DM component, even after taking the high scale validity constraints. We exemplified this at LHC for hadronically quiet dilepton channel with missing energy, where $\Delta M$, turns out to be a crucial kinematic parameter, constrained from DM, high scale validity and neutrino sector. At LHC, due to its $t \bar{t}$ background, high $\Delta M$ regions can be segregated from SM background more efficiently at the cost of small production cross section. Low $\Delta M$ regions are more affected by SM background, although the signal cross-section is high. Therefore the model can be probed within High Luminosity reach of LHC. On the other hand, $e^{+} e^{-}$annihilation have better possibility to explore low $\Delta M$ regions absent $t \bar{t}$ background. Here, we would like to comment that there are several studies that have been done in this direction, but the high scale validity constraint may alter the conclusion significantly as we demonstrate.

Finally, we would like to mention that the analysis performed here, although focus on a specific model set up, but there are some generic conclusions that can be borrowed. For example, if the two DM components have sufficient interaction in between, the available parameter space will be enlarged significantly from both relic density and direct search. 
The conversion of one DM into the other may also affect the collider outcome of the DM significantly. It is obvious that richer signal is obtained when we have larger multiplets in dark sector (as scalar doublet produces two lepton final state in the analysis). It is also possible that the dark sector and neutrino sector although may not inherit a common origin, the high scale validity of the model can bring them together.

\section{Acknowledgments}

PG would like to thank Basabendu Barman and Rishav Roshan for useful discussions and also acknowledges MHRD, Government of India for research fellowship. SB would like to acknowledge the DST-INSPIRE research grant IFA13-PH-57 at IIT Guwahati.

\section{A Tree level unitarity constraints}

In this section, we perform the analysis to find the tree level unitarity limits on quartic couplings present in our model at high energy. The scattering amplitude for any $2 \rightarrow 2$ process can be expressed in terms of the Legendre polynomial as [122]

$$
\mathcal{M}^{2 \rightarrow 2}=16 \pi \sum_{l=0}^{\infty} a_{l}(2 l+1) P_{l}(\cos \theta),
$$

where $\theta$ is the scattering angle and $P_{l}(\cos \theta)$ is the Legendre polynomial of order $l$. In the high-energy limit, only the s-wave $(l=0)$ partial amplitude $a_{0}$ will determine the leading energy dependence of the scattering processes. The unitarity constraint turns out to be $[72,73,122]$

$$
\operatorname{Re}\left|a_{0}\right|<\frac{1}{2}
$$

The constraint in eq. (A.2) can be further converted to a bound on the scattering amplitude $\mathcal{M}[72,73,122]$ :

$$
|\mathcal{M}|<8 \pi \text {. }
$$

In the present set up, we have multiple possible $2 \rightarrow 2$ scattering processes. Therefore, we need to construct a matrix $\left(M_{i ; j}^{2 \rightarrow 2}=\mathcal{M}_{i \rightarrow j}\right)$ by considering all possible two particle states. Finally, we calculate the eigenvalues of $\mathcal{M}$ and employ the bound as in eq. (A.3). In the high-energy limit, we express the SM Higgs doublet as $H^{T}=\left(w^{+} \frac{h+i z}{\sqrt{2}}\right)$. Then, the scalar potential in eq. (2.2) gives rise to 19 neutral combinations of two particle states:

$$
\begin{array}{r}
w^{+} w^{-}, H^{+} H^{-}, \frac{h h}{\sqrt{2}}, \frac{z z}{\sqrt{2}}, \frac{H^{0} H^{0}}{\sqrt{2}}, \frac{A^{0} A^{0}}{\sqrt{2}}, \frac{\phi \phi}{\sqrt{2}}, h z, H^{0} A^{0}, w^{+} H^{-}, H^{+} w^{-}, \\
h H^{0}, h A^{0}, z H^{0}, z A^{0}, h \phi, z \phi, H^{0} \phi, A^{0} \phi,
\end{array}
$$

and 10 singly charged two-particle states:

$$
h w^{+}, z w^{+}, H^{0} H^{+}, A^{0} H^{+}, h H^{+}, z H^{+}, H^{0} w^{+}, A^{0} w^{+}, \phi w^{+}, \phi H^{+} .
$$


Therefore, we can write the scattering amplitude matrix (M) in block-diagonal form by decomposing it into a neutral $(N C)$ and singly charged $(S C)$ sector as

$$
M=\left(\begin{array}{cc}
M_{19 \times 19}^{\mathrm{NC}} & 0 \\
0 & M_{10 \times 10}^{\mathrm{SC}}
\end{array}\right)
$$

where the sub-matrices are given by

$$
M_{19 \times 19}^{\mathrm{NC}}=\left(\begin{array}{cccc}
\left(M_{1}^{\mathrm{NC}}\right)_{7 \times 7} & 0 & 0 & 0 \\
0 & \left(M_{2}^{\mathrm{NC}}\right)_{2 \times 2} & 0 & 0 \\
0 & 0 & \left(M_{3}^{\mathrm{NC}}\right)_{6 \times 6} & 0 \\
0 & 0 & 0 & \left(M_{4}^{\mathrm{NC}}\right)_{4 \times 4}
\end{array}\right)
$$

with

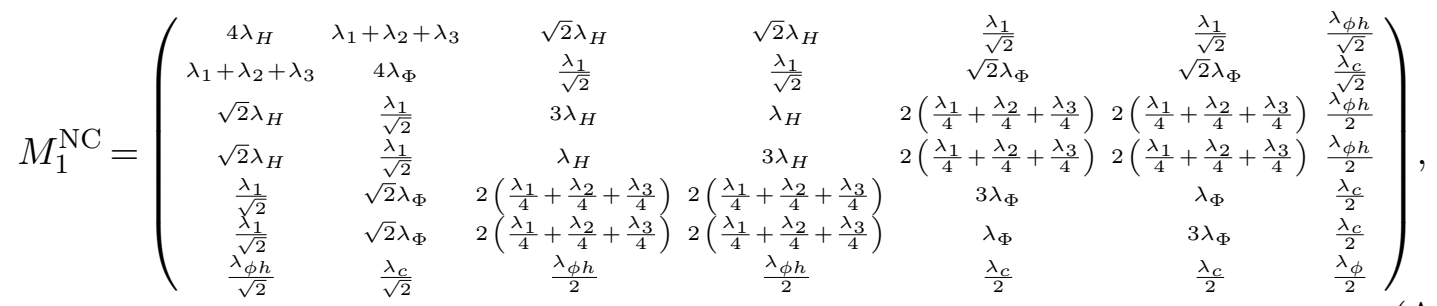

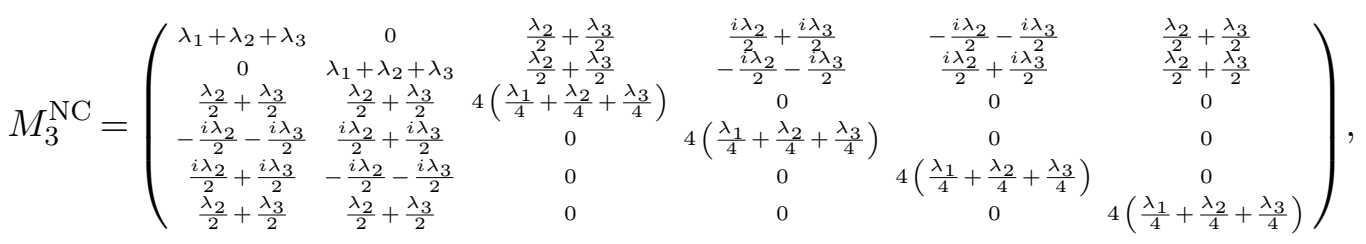

$$
\begin{aligned}
& M_{2}^{\mathrm{NC}}=\left(\begin{array}{cc}
2 \lambda_{H} & 0 \\
0 & 2 \lambda_{\Phi}
\end{array}\right), \quad M_{4}^{\mathrm{NC}}=\left(\begin{array}{cccc}
\lambda_{\phi h} & 0 & 0 & 0 \\
0 & \lambda_{\phi h} & 0 & 0 \\
0 & 0 & \lambda_{c} & 0 \\
0 & 0 & 0 & \lambda_{c}
\end{array}\right) \text {, }
\end{aligned}
$$

and

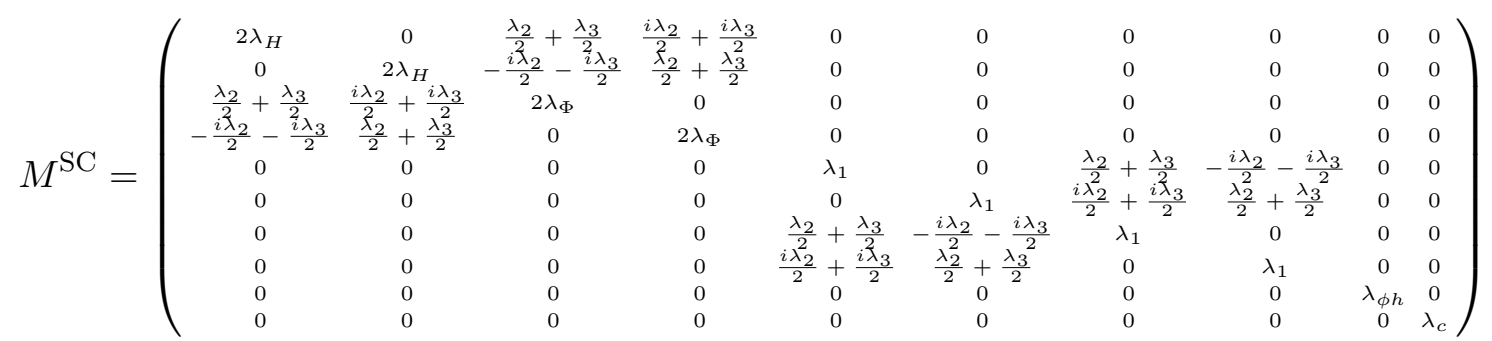



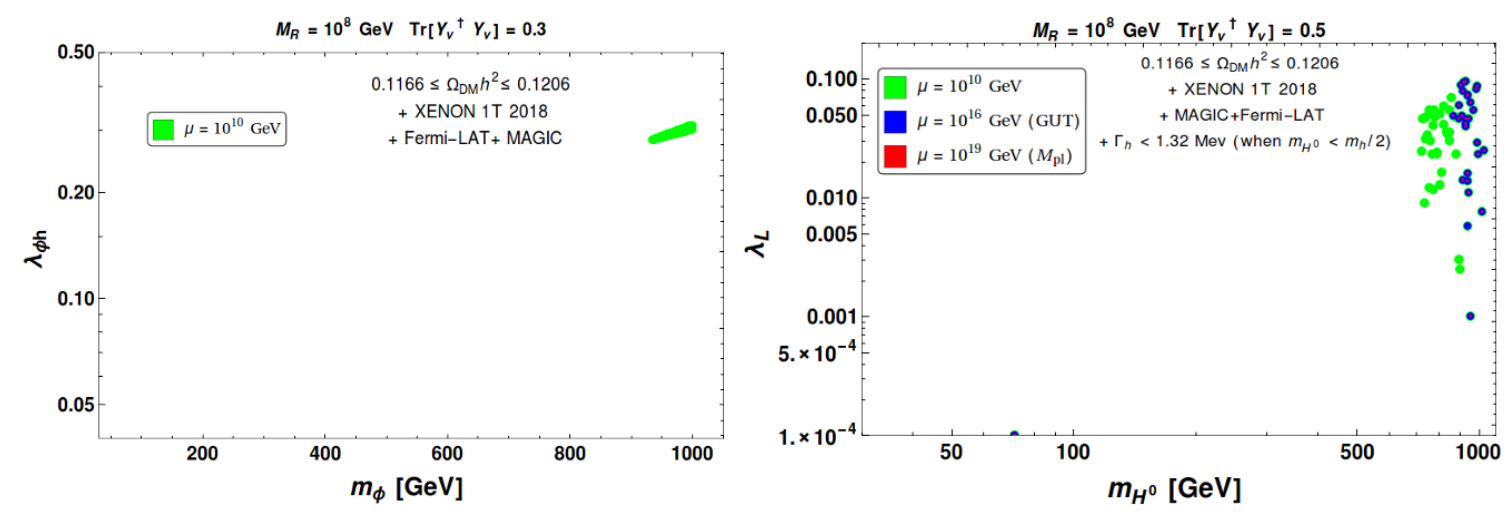

Figure 32. Parameter space scan for (left) scalar singlet DM and (right) IDM cosnidering satisfaction of relic density bound, direct detection cross section limit, indirect cross-section limit and high scale validity in presence of RH neutrinos.

After determining the eigenvalues of eq. (A.6) we conclude that tree level unitarity constraints in this set up are following:

$$
\begin{array}{rlrl}
\left|\lambda_{H}\right| & <4 \pi, & \left|\lambda_{\Phi}\right| & <4 \pi, \\
\left|\lambda_{c}\right| & <8 \pi, & \left|\lambda_{\phi h}\right| & <8 \pi, \\
\left|\lambda_{1}\right| & <8 \pi, & \left|\lambda_{1}+2\left(\lambda_{2}+\lambda_{3}\right)\right| & <8 \pi \\
\left|\lambda_{1}+\lambda_{2}+\lambda_{3}\right| & <8 \pi, & \left|\lambda_{1}-\lambda_{2}-\lambda_{3}\right| & <8 \pi, \\
\left|\left(\lambda_{\Phi}+\lambda_{H}\right) \pm \sqrt{\left(\lambda_{2}+\lambda_{3}\right)^{2}+\left(\lambda_{H}-\lambda_{\Phi}\right)^{2}}\right| & <8 \pi,
\end{array}
$$

and $\left|x_{1,2,3}\right|<16 \pi$

where $x_{1,2,3}$ be the roots of following cubic equation

$$
\begin{array}{r}
x^{3}+x^{2}\left(-12 \lambda_{H}-12 \lambda_{\Phi}-\lambda_{\phi}\right)+x\left(-16 \lambda_{1}^{2}-16 \lambda_{1} \lambda_{2}-16 \lambda_{1} \lambda_{3}-4 \lambda_{2}^{2}-8 \lambda_{2} \lambda_{3}\right. \\
\left.-4 \lambda_{3}^{2}-4 \lambda_{c}^{2}+144 \lambda_{H} \lambda_{\Phi}+12 \lambda_{H} \lambda_{\phi}+12 \lambda_{\Phi} \lambda_{\phi}-4 \lambda_{\phi h}^{2}\right)+16 \lambda_{1}^{2} \lambda_{\phi}+16 \lambda_{1} \lambda_{2} \lambda_{\phi} \\
+16 \lambda_{1} \lambda_{3} \lambda_{\phi}-32 \lambda_{1} \lambda_{c} \lambda_{\phi h}+4 \lambda_{2}^{2} \lambda_{\phi}+8 \lambda_{2} \lambda_{3} \lambda_{\phi}-16 \lambda_{2} \lambda_{c} \lambda_{\phi h}+4 \lambda_{3}^{2} \lambda_{\phi} \\
-16 \lambda_{3} \lambda_{c} \lambda_{\phi h}+48 \lambda_{c}^{2} \lambda_{H}-144 \lambda_{H} \lambda_{\Phi} \lambda_{\phi}+48 \lambda_{\Phi} \lambda_{\phi h}^{2}=0
\end{array}
$$

\section{B High scale validity of single component DM models}

Here we show the allowed parameter space for single component DM when the vacuum stability conditions are taken into account in presence of $\mathrm{RH}$ neutrinos. In left panel of figure 32 the parameter space for scalar singlet DM $(\phi)$ is shown considering $M_{R}=10^{8} \mathrm{GeV}$ and $\operatorname{Tr}\left[Y_{\nu}^{\dagger} Y_{\nu}\right]=0.3$, while in right panel we present the same for IDM $\left(H^{0}\right)$ with $M_{R}=$ $10^{8} \mathrm{GeV}$ and $\operatorname{Tr}\left[Y_{\nu}^{\dagger} Y_{\nu}\right]=0.5$. We see that for $\phi$, the DM mass is allowed beyond $900 \mathrm{GeV}$ considering the absolute stability of the EW vacuum upto $10^{10} \mathrm{GeV}$ [93]. However for IDM, we notice that the absolute EW vacuum stability can be extended even upto Planck scale (with similar Yukawa) due to the presence of several scalar degrees of freedom. 


\begin{tabular}{|c|c|c|c|c|c|}
\hline$m_{\phi}>m_{H^{0}}($ in $\mathrm{GeV})$ & \multicolumn{2}{|c|}{ RH Neutrinos } & \multicolumn{3}{|c|}{ Relic + DD (XENON 1T) + ID (Fermi LAT+MAGIC) + Stability + Perturbativity } \\
\hline Relic + DD (XENON 1T)+ID (Fermi LAT+MAGIC) & $M_{R}$ & $\operatorname{Tr}\left[Y_{\nu}^{\dagger} Y_{\nu}\right]$ & $\mu=10^{10} \mathrm{GeV}$ & $\mu=10^{16} \mathrm{GeV}(\mathrm{GUT})$ & $\mu=10^{19} \mathrm{GeV}\left(M_{\mathrm{pl}}\right)$ \\
\hline \multirow{6}{*}{$\begin{aligned} & m_{H^{0}} \sim\{85-500\} \\
& m_{\phi} \sim\{170-500\} \\
& \Delta m \equiv m_{A^{0}}-m_{H^{0}} \sim\{0-200\} \\
& \Delta M \equiv m_{H^{ \pm}}-m_{H^{0}} \sim\{4-360\} \\
& \lambda_{c} \sim\{0.08-1.00\} \\
& \lambda_{L} \sim\{0.001-0.28\} \\
& \quad \lambda_{\phi h} \sim\{0.001-0.14\}\end{aligned}$} & \multirow[t]{2}{*}{$\begin{array}{c}10^{4} \\
\mathrm{GeV}\end{array}$} & 0.1 & $\begin{aligned} m_{H^{0}} & \sim\{88-500\} \\
m_{\phi} & \sim\{170-500\} \\
\Delta m & \sim\{0-90\} \\
\Delta M & \sim\{5-106\} \\
\lambda_{c} & \sim\{0.086-0.70\} \\
\lambda_{L} & \sim\{0.001-0.09\} \\
\lambda_{\phi h} & \sim\{0.001-0.14\}\end{aligned}$ & $\begin{aligned} m_{H^{0}} & \sim\{94-496\} \\
m_{\phi} & \sim\{220-500\} \\
\Delta m & \sim\{0-52\} \\
\Delta M & \sim\{6-70\} \\
\lambda_{c} & \sim\{0.10-0.52\} \\
\lambda_{L} & \sim\{0.001-0.054\} \\
\lambda_{\phi h} & \sim\{0.001-0.14\}\end{aligned}$ & $\begin{aligned} & m_{H^{0}} \sim\{94-496\} \\
& m_{\phi} \sim\{220-500\} \\
& \Delta m \sim\{0-38\} \\
& \Delta M \sim\{6-55\} \\
& \lambda_{c} \sim\{0.10-0.52\} \\
& \lambda_{L} \sim\{0.001-0.046\} \\
& \lambda_{\phi h} \sim\{0.001-0.14\}\end{aligned}$ \\
\hline & & 0.9 & $\begin{aligned} m_{H^{0}} & \sim\{89-493\} \\
m_{\phi} & \sim\{258-500\} \\
\Delta m & \sim\{0-87\} \\
\Delta M & \sim\{21-106\} \\
\lambda_{c} & \sim\{0.086-0.69\} \\
\lambda_{L} & \sim\{0.001-0.09\} \\
\lambda_{\phi h} & \sim\{0.001-0.11\}\end{aligned}$ & $\begin{array}{c}\text { No parameter space } \\
\text { avialable }\end{array}$ & $\begin{array}{c}\text { No parameter space } \\
\text { avialable }\end{array}$ \\
\hline & \multirow[t]{2}{*}{$\begin{array}{l}10^{8} \\
\mathrm{GeV}\end{array}$} & 0.1 & $\begin{aligned} m_{H^{0}} & \sim\{85-496\} \\
m_{\phi} & \sim\{171-500\} \\
\Delta m & \sim\{0-87\} \\
\Delta M & \sim\{4-123\} \\
\lambda_{c} & \sim\{0.086-0.69\} \\
\lambda_{L} & \sim\{0.001-0.09\} \\
\lambda_{\phi h} & \sim\{0.001-0.14\}\end{aligned}$ & $\begin{aligned} m_{H^{0}} & \sim\{94-496\} \\
m_{\phi} & \sim\{221-500\} \\
\Delta m & \sim\{0-52\} \\
\Delta M & \sim\{6-69\} \\
\lambda_{c} & \sim\{0.10-0.52\} \\
\lambda_{L} & \sim\{0.001-0.06\} \\
\lambda_{\phi h} & \sim\{0.001-0.14\}\end{aligned}$ & $\begin{aligned} m_{H^{0}} & \sim\{94-496\} \\
m_{\phi} & \sim\{221-500\} \\
\Delta m & \sim\{0-38\} \\
\Delta M & \sim\{6-55\} \\
\lambda_{c} & \sim\{0.10-0.52\} \\
\lambda_{L} & \sim\{0.001-0.046\} \\
\lambda_{\phi h} & \sim\{0.001-0.14\}\end{aligned}$ \\
\hline & & 0.9 & $\begin{aligned} m_{H^{0}} & \sim\{89-493\} \\
m_{\phi} & \sim\{171-500\} \\
\Delta m & \sim\{0-87\} \\
\Delta M & \sim\{17-106\} \\
\lambda_{c} & \sim\{0.086-0.69\} \\
\lambda_{L} & \sim\{0.001-0.09\} \\
\lambda_{\phi h} & \sim\{0.001-0.10\}\end{aligned}$ & $\begin{aligned} m_{H^{0}} & \sim\{98-432\} \\
m_{\phi} & \sim\{317-498\} \\
\Delta m & \sim\{1-52\} \\
\Delta M & \sim\{19-69\} \\
\lambda_{c} & \sim\{0.10-0.40\} \\
\lambda_{L} & \sim\{0.001-0.046\} \\
\lambda_{\phi h} & \sim\{0.001-0.096\}\end{aligned}$ & $\begin{array}{c}\text { No parameter space } \\
\text { avialable }\end{array}$ \\
\hline & \multirow[t]{2}{*}{$\begin{array}{l}10^{12} \\
\mathrm{GeV}\end{array}$} & 0.1 & $\begin{array}{c}\text { No effective contribution } \\
\text { from } \\
\text { RH Neutrinos }\end{array}$ & $\begin{aligned} m_{H^{0}} & \sim\{94-496\} \\
m_{\phi} & \sim\{221-500\} \\
\Delta m & \sim\{0-52\} \\
\Delta M & \sim\{6-69\} \\
\lambda_{c} & \sim\{0.10-0.52\} \\
\lambda_{L} & \sim\{0.001-0.06\} \\
\lambda_{\phi h} & \sim\{0.001-0.14\}\end{aligned}$ & $\begin{aligned} & m_{H^{0}} \sim\{94-496\} \\
& m_{\phi} \sim\{221-500\} \\
& \Delta m \sim\{0-38\} \\
& \Delta M \sim\{6-55\} \\
& \lambda_{c} \sim\{0.10-0.52\} \\
& \lambda_{L} \sim\{0.001-0.046\} \\
& \lambda_{\phi h} \sim\{0.001-0.14\}\end{aligned}$ \\
\hline & & 0.9 & $\begin{array}{c}\text { No effective contribution } \\
\text { from } \\
\text { RH Neutrinos }\end{array}$ & $\begin{aligned} m_{H^{0}} & \sim\{98-493\} \\
m_{\phi} & \sim\{248-498\} \\
\Delta m & \sim\{0-52\} \\
\Delta M & \sim\{17-69\} \\
\lambda_{c} & \sim\{0.10-0.52\} \\
\lambda_{L} & \sim\{0.001-0.054\} \\
\lambda_{\phi h} & \sim\{0.001-0.096\}\end{aligned}$ & $\begin{aligned} m_{H^{0}} & \sim\{129-493\} \\
m_{\phi} & \sim\{271-495\} \\
\Delta m & \sim\{0-37\} \\
\Delta M & \sim\{17-55\} \\
\lambda_{c} & \sim\{0.10-0.25\} \\
\lambda_{L} & \sim\{0.003-0.046\} \\
\lambda_{\phi h} & \sim\{0.007-0.092\}\end{aligned}$ \\
\hline
\end{tabular}

Table 9. Allowed ranges of relevant parameters considering $m_{\phi}>m_{H^{0}}$ for different values of RH neutrino mass and $\operatorname{Tr}\left[Y_{\nu}^{\dagger} Y_{\nu}\right]$.

\section{Available parameters of the model from high scale validity for different choices of RH neutrino mass and Yukawa coupling}

Here, we would like to show the available parameter space of the two component DM model with RH neutrinos, viable from high scale validity constraint after choosing different possible RH neutrino mass and Neutrino Yukawa coupling together with relic density, direct search and indirect search bound. In the main text, we elaborated only the case of $\operatorname{Tr}\left[Y_{\nu}^{\dagger} Y_{\nu}\right]=0.5$ with RH neutrino mass of $10^{8} \mathrm{GeV}$. We show different possibilities in two tables, in table 9 , we depict the case of $m_{\phi}>m_{H^{0}}$ and in table 10, the reverse hierarchy $m_{\phi} \leq m_{H^{0}}$ is presented. 


\begin{tabular}{|c|c|c|c|c|c|}
\hline$m_{\phi} \leq m_{H^{0}}($ in $\mathrm{GeV})$ & \multicolumn{2}{|c|}{ RH Neutrinos } & \multicolumn{3}{|c|}{ Relic + DD (XENON 1T) +ID (Fermi LAT+MAGIC) + Stability + Perturbativity } \\
\hline Relic + DD (XENON 1T)+ID (Fermi LAT+MAGIC) & $M_{R}$ & $\operatorname{Tr}\left[Y_{\nu}^{\dagger} Y_{\nu}\right]$ & $\mu=10^{10} \mathrm{GeV}$ & $\mu=10^{16} \mathrm{GeV}(\mathrm{GUT})$ & $\mu=10^{19} \mathrm{GeV}\left(M_{\mathrm{pl}}\right)$ \\
\hline \multirow{5}{*}{$\begin{aligned} & m_{H^{0}} \sim\{100-500\} \\
& m_{\phi} \sim\{96-500\} \\
& \Delta m \equiv m_{A^{0}}-m_{H^{0}} \sim\{0-200\} \\
& \Delta M \equiv m_{H^{ \pm}}-m_{H^{0}} \sim\{12-300\} \lambda_{c} \sim\{0.30-1.00\} \\
& \lambda_{L} \sim\{0.001-0.30\} \\
& \lambda_{\phi h} \sim\{0.001-0.11\}\end{aligned}$} & \multirow[t]{2}{*}{$\begin{array}{l}10^{4} \\
\mathrm{GeV}\end{array}$} & 0.1 & $\begin{aligned} & m_{H^{0}} \sim\{100-479\} \\
& m_{\phi} \sim\{96-471\} \\
& \Delta m \sim\{0-57\} \\
& \Delta M \sim\{12-78\} \\
& \lambda_{c} \sim\{0.34-0.98\} \\
& \lambda_{L} \sim\{0.001-0.127\} \\
& \lambda_{\phi h} \sim\{0.001-0.096\}\end{aligned}$ & $\begin{aligned} & m_{H^{0}} \sim\{205-452\} \\
& m_{\phi} \sim\{201-451\} \\
& \Delta m \sim\{5-11\} \\
& \Delta M \sim\{12-29\} \\
& \lambda_{c} \sim\{0.43-0.81\} \\
& \lambda_{L} \sim\{0.008-0.043\} \\
& \lambda_{\phi h} \sim\{0.002-0.043\}\end{aligned}$ & $\begin{aligned} m_{H^{0}} & \sim\{205-225\} \\
m_{\phi} & \sim\{201-224\} \\
\Delta m & \sim\{5-11\} \\
\Delta M & \sim\{16-29\} \\
\lambda_{c} & \sim\{0.43-0.59\} \\
\lambda_{L} & \sim\{0.024-0.025\} \\
\lambda_{\phi h} & \sim\{0.002-0.016\}\end{aligned}$ \\
\hline & & 0.9 & $\begin{aligned} m_{H^{0}} & \sim\{170-455\} \\
m_{\phi} & \sim\{168-450\} \\
\Delta m & \sim\{0-57\} \\
\Delta M & \sim\{26-67\} \\
\lambda_{c} & \sim\{0.34-0.98\} \\
\lambda_{L} & \sim\{0.001-0.054\} \\
\lambda_{\phi h} & \sim\{0.004-0.086\}\end{aligned}$ & $\begin{array}{c}\text { No parameter space } \\
\text { available }\end{array}$ & $\begin{array}{c}\text { No parameter space } \\
\text { avialable }\end{array}$ \\
\hline & \multirow[t]{2}{*}{$\begin{array}{c}10^{8} \\
\mathrm{GeV}\end{array}$} & 0.1 & $\begin{aligned} m_{H^{0}} & \sim\{100-479\} \\
m_{\phi} & \sim\{96-471\} \\
\Delta m & \sim\{0-57\} \\
\Delta M & \sim\{12-78\} \\
\lambda_{c} & \sim\{0.34-0.98\} \\
\lambda_{L} & \sim\{0.001-0.127\} \\
\lambda_{\phi h} & \sim\{0.001-0.096\}\end{aligned}$ & $\begin{aligned} & m_{H^{0}} \sim\{126-452\} \\
& m_{\phi} \sim\{122-451\} \\
& \Delta m \sim\{0-19\} \\
& \Delta M \sim\{12-29\} \\
& \lambda_{c} \sim\{0.43-0.81\} \\
& \lambda_{L} \sim\{0.004-0.043\} \\
& \lambda_{\phi h} \sim\{0.002-0.054\}\end{aligned}$ & $\begin{aligned} m_{H^{0}} & \sim\{126-224\} \\
m_{\phi} & \sim\{122-224\} \\
\Delta m & \sim\{0-19\} \\
\Delta M & \sim\{16-29\} \\
\lambda_{c} & \sim\{0.43-0.61\} \\
\lambda_{L} & \sim\{0.004-0.025\} \\
\lambda_{\phi h} & \sim\{0.002-0.016\}\end{aligned}$ \\
\hline & & 0.9 & $\begin{aligned} m_{H^{0}} & \sim\{100-479\} \\
m_{\phi} & \sim\{96-471\} \\
\Delta m & \sim\{0-57\} \\
\Delta M & \sim\{19-78\} \\
\lambda_{c} & \sim\{0.34-0.98\} \\
\lambda_{L} & \sim\{0.001-0.127\} \\
\lambda_{\phi h} & \sim\{0.001-0.096\}\end{aligned}$ & $\begin{array}{c}\text { No parameter space } \\
\text { available }\end{array}$ & $\begin{array}{c}\text { No parameter space } \\
\text { available }\end{array}$ \\
\hline & $\begin{array}{l}10^{12} \\
\mathrm{GeV}\end{array}$ & 0.1 & $\begin{array}{c}\text { No effective contribution } \\
\text { from } \\
\text { RH Neutrinos }\end{array}$ & $\begin{aligned} & m_{H^{0}} \sim\{126-452\} \\
& m_{\phi} \sim\{122-451\} \\
& \Delta m \sim\{5-19\} \\
& \Delta M \sim\{12-29\} \\
& \lambda_{c} \sim\{0.43-0.81\} \\
& \lambda_{L} \sim\{0.004-0.043\} \\
& \lambda_{\phi h} \sim\{0.002-0.054\}\end{aligned}$ & $\begin{aligned} m_{H^{0}} & \sim\{126-225\} \\
m_{\phi} & \sim\{122-224\} \\
\Delta m & \sim\{5-19\} \\
\Delta M & \sim\{16-29\} \\
\lambda_{c} & \sim\{0.43-0.61\} \\
\lambda_{L} & \sim\{0.004-0.025\} \\
\lambda_{\phi h} & \sim\{0.002-0.016\}\end{aligned}$ \\
\hline
\end{tabular}

Table 10. Allowed ranges of relevant parameters considering $m_{\phi} \leq m_{H^{0}}$ for different values of $\mathrm{RH}$ neutrino mass and $\operatorname{Tr}\left[Y_{\nu}^{\dagger} Y_{\nu}\right]$.

Open Access. This article is distributed under the terms of the Creative Commons Attribution License (CC-BY 4.0), which permits any use, distribution and reproduction in any medium, provided the original author(s) and source are credited.

\section{References}

[1] CMS collaboration, Observation of a New Boson at a Mass of 125 GeV with the CMS Experiment at the LHC, Phys. Lett. B 716 (2012) 30 [arXiv:1207.7235] [InSPIRE].

[2] ATLAS collaboration, Observation of a new particle in the search for the Standard Model Higgs boson with the ATLAS detector at the LHC, Phys. Lett. B 716 (2012) 1 [arXiv:1207.7214] [INSPIRE].

[3] S. Alekhin, A. Djouadi and S. Moch, The top quark and Higgs boson masses and the stability of the electroweak vacuum, Phys. Lett. B 716 (2012) 214 [arXiv:1207.0980] [InSPIRE].

[4] D. Buttazzo et al., Investigating the near-criticality of the Higgs boson, JHEP 12 (2013) 089 [arXiv: 1307.3536] [INSPIRE].

[5] G. Isidori, G. Ridolfi and A. Strumia, On the metastability of the standard model vacuum, Nucl. Phys. B 609 (2001) 387 [hep-ph/0104016] [INSPIRE]. 
[6] L.A. Anchordoqui et al., Vacuum Stability of Standard Model ${ }^{++}$, JHEP 02 (2013) 074 [arXiv:1208.2821] [INSPIRE].

[7] Y. Tang, Vacuum Stability in the Standard Model, Mod. Phys. Lett. A 28 (2013) 1330002 [arXiv:1301.5812] [INSPIRE].

[8] J. Ellis, J.R. Espinosa, G.F. Giudice, A. Hoecker and A. Riotto, The Probable Fate of the Standard Model, Phys. Lett. B 679 (2009) 369 [arXiv:0906.0954] [INSPIRE].

[9] J. Elias-Miro, J.R. Espinosa, G.F. Giudice, G. Isidori, A. Riotto and A. Strumia, Higgs mass implications on the stability of the electroweak vacuum, Phys. Lett. B 709 (2012) 222 [arXiv:1112.3022] [INSPIRE].

[10] Particle Data Group collaboration, Review of Particle Physics, Phys. Rev. D 98 (2018) 030001 [INSPIRE].

[11] J. Elias-Miro, J.R. Espinosa, G.F. Giudice, H.M. Lee and A. Strumia, Stabilization of the Electroweak Vacuum by a Scalar Threshold Effect, JHEP 06 (2012) 031 [arXiv:1203.0237] [INSPIRE].

[12] O. Lebedev, On stability of the Electroweak Vacuum and the Higgs Portal, Eur. Phys. J. C 72 (2012) 2058 [arXiv:1203.0156] [INSPIRE].

[13] WMAP collaboration, Wilkinson Microwave Anisotropy Probe (WMAP) three year results: implications for cosmology, Astrophys. J. Suppl. 170 (2007) 377 [astro-ph/0603449] [INSPIRE].

[14] Planck collaboration, Planck 2018 results. VI. Cosmological parameters, arXiv: 1807.06209 [INSPIRE].

[15] L. Roszkowski, E.M. Sessolo and S. Trojanowski, WIMP dark matter candidates and searches - current status and future prospects, Rept. Prog. Phys. 81 (2018) 066201 [arXiv: 1707.06277] [INSPIRE].

[16] V. Silveira and A. Zee, Scalar phantoms, Phys. Lett. 161B (1985) 136 [inSPIRE].

[17] J. McDonald, Gauge singlet scalars as cold dark matter, Phys. Rev. D 50 (1994) 3637 [hep-ph/0702143] [INSPIRE].

[18] J.M. Cline, K. Kainulainen, P. Scott and C. Weniger, Update on scalar singlet dark matter, Phys. Rev. D 88 (2013) 055025 [Erratum ibid. D 92 (2015) 039906] [arXiv:1306.4710] [INSPIRE].

[19] W.-L. Guo and Y.-L. Wu, The real singlet scalar dark matter model, JHEP 10 (2010) 083 [arXiv: 1006.2518] [INSPIRE].

[20] L. Feng, S. Profumo and L. Ubaldi, Closing in on singlet scalar dark matter: LUX, invisible Higgs decays and gamma-ray lines, JHEP 03 (2015) 045 [arXiv: 1412.1105] [INSPIRE].

[21] S. Bhattacharya, S. Jana and S. Nandi, Neutrino Masses and Scalar Singlet Dark Matter, Phys. Rev. D 95 (2017) 055003 [arXiv: 1609.03274] [INSPIRE].

[22] J.A. Casas, D.G. Cerdeño, J.M. Moreno and J. Quilis, Reopening the Higgs portal for single scalar dark matter, JHEP 05 (2017) 036 [arXiv:1701.08134] [INSPIRE].

[23] S. Bhattacharya, P. Ghosh, T.N. Maity and T.S. Ray, Mitigating Direct Detection Bounds in Non-minimal Higgs Portal Scalar Dark Matter Models, JHEP 10 (2017) 088 [arXiv: 1706. 04699] [INSPIRE]. 
[24] S. Bhattacharya, P. Ghosh and S. Verma, SIMPler realisation of Scalar Dark Matter, JCAP 01 (2020) 040 [arXiv: 1904.07562] [INSPIRE].

[25] LUX collaboration, Results from a search for dark matter in the complete LUX exposure, Phys. Rev. Lett. 118 (2017) 021303 [arXiv: 1608.07648] [INSPIRE].

[26] XENON collaboration, First Dark Matter Search Results from the XENON1T Experiment, Phys. Rev. Lett. 119 (2017) 181301 [arXiv:1705.06655] [INSPIRE].

[27] XENON collaboration, Latest results of 1 tonne $\times$ year Dark Matter Search with XENON1T, PoS(EDSU2018) 017.

[28] PandaX collaboration, Dark matter direct search sensitivity of the PandaX-4T experiment, Sci. China Phys. Mech. Astron. 62 (2019) 31011 [arXiv:1806.02229] [INSPIRE].

[29] S. Bhattacharya, A. Drozd, B. Grzadkowski and J. Wudka, Two-Component Dark Matter, JHEP 10 (2013) 158 [arXiv:1309.2986] [INSPIRE].

[30] L. Bian, R. Ding and B. Zhu, Two Component Higgs-Portal Dark Matter, Phys. Lett. B 728 (2014) 105 [arXiv:1308.3851] [InSPIRE].

[31] S. Esch, M. Klasen and C.E. Yaguna, A minimal model for two-component dark matter, JHEP 09 (2014) 108 [arXiv:1406.0617] [InSPIRE].

[32] A. Karam and K. Tamvakis, Dark matter and neutrino masses from a scale-invariant multi-Higgs portal, Phys. Rev. D 92 (2015) 075010 [arXiv:1508.03031] [INSPIRE].

[33] A. Karam and K. Tamvakis, Dark Matter from a Classically Scale-Invariant $\mathrm{SU}(3)_{X}$, Phys. Rev. D 94 (2016) 055004 [arXiv: 1607.01001] [inSPIRE].

[34] A. Ahmed, M. Duch, B. Grzadkowski and M. Iglicki, Multi-Component Dark Matter: the vector and fermion case, Eur. Phys. J. C 78 (2018) 905 [arXiv:1710.01853] [INSPIRE].

[35] J. Herrero-Garcia, A. Scaffidi, M. White and A.G. Williams, On the direct detection of multi-component dark matter: implications of the relic abundance, JCAP 01 (2019) 008 [arXiv: 1809.06881] [INSPIRE].

[36] A. Poulin and S. Godfrey, Multicomponent dark matter from a hidden gauged SU(3), Phys. Rev. D 99 (2019) 076008 [arXiv: 1808.04901] [INSPIRE].

[37] M. Aoki and T. Toma, Boosted Self-interacting Dark Matter in a Multi-component Dark Matter Model, JCAP 10 (2018) 020 [arXiv:1806.09154] [INSPIRE].

[38] M. Aoki, D. Kaneko and J. Kubo, Multicomponent Dark Matter in Radiative Seesaw Models, Front. in Phys. 5 (2017) 53 [arXiv:1711.03765] [INSPIRE].

[39] S. Bhattacharya, P. Poulose and P. Ghosh, Multipartite Interacting Scalar Dark Matter in the light of updated LUX data, JCAP 04 (2017) 043 [arXiv: 1607.08461] [INSPIRE].

[40] S. Bhattacharya, P. Ghosh and N. Sahu, Multipartite Dark Matter with Scalars, Fermions and signatures at LHC, JHEP 02 (2019) 059 [arXiv: 1809.07474] [INSPIRE].

[41] A. Biswas, D. Majumdar, A. Sil and P. Bhattacharjee, Two Component Dark Matter: A Possible Explanation of $130 \mathrm{GeV} \gamma-$ Ray Line from the Galactic Centre, JCAP 12 (2013) 049 [arXiv: 1301.3668] [INSPIRE].

[42] D. Borah, R. Roshan and A. Sil, Minimal two-component scalar doublet dark matter with radiative neutrino mass, Phys. Rev. D 100 (2019) 055027 [arXiv:1904.04837] [INSPIRE]. 
[43] S. Chakraborti and P. Poulose, Interplay of Scalar and Fermionic Components in a Multi-component Dark Matter Scenario, Eur. Phys. J. C 79 (2019) 420 [arXiv:1808.01979] [INSPIRE].

[44] S. Chakraborti, A. Dutta Banik and R. Islam, Probing Multicomponent Extension of Inert Doublet Model with a Vector Dark Matter, Eur. Phys. J. C 79 (2019) 662 [arXiv:1810.05595] [INSPIRE].

[45] B. Barman, S. Bhattacharya and M. Zakeri, Multipartite Dark Matter in $\mathrm{SU}(2)_{N}$ extension of Standard Model and signatures at the LHC, JCAP 09 (2018) 023 [arXiv:1806.01129] [INSPIRE].

[46] A. Dutta Banik, M. Pandey, D. Majumdar and A. Biswas, Two component WIMP-FImP dark matter model with singlet fermion, scalar and pseudo scalar, Eur. Phys. J. C 77 (2017) 657 [arXiv:1612.08621] [INSPIRE].

[47] S. Bhattacharya, A.K. Saha, A. Sil and J. Wudka, Dark Matter as a remnant of SQCD Inflation, JHEP 10 (2018) 124 [arXiv: 1805.03621] [INSPIRE].

[48] S. Yaser Ayazi and A. Mohamadnejad, Scale-Invariant Two Component Dark Matter, Eur. Phys. J. C 79 (2019) 140 [arXiv:1808.08706] [INSPIRE].

[49] J. Abdallah et al., Simplified Models for Dark Matter Searches at the LHC, Phys. Dark Univ. 9-10 (2015) 8.

[50] D. Abercrombie et al., Dark Matter Benchmark Models for Early LHC Run-2 Searches: Report of the ATLAS/CMS Dark Matter Forum, Phys. Dark Univ. 26 (2019) 100371 [arXiv: 1507.00966] [INSPIRE].

[51] H. Han, J.M. Yang, Y. Zhang and S. Zheng, Collider Signatures of Higgs-portal Scalar Dark Matter, Phys. Lett. B 756 (2016) 109 [arXiv:1601.06232] [INSPIRE].

[52] M. Gustafsson, S. Rydbeck, L. Lopez-Honorez and E. Lundstrom, Status of the Inert Doublet Model and the Role of multileptons at the LHC, Phys. Rev. D 86 (2012) 075019 [arXiv:1206.6316] [INSPIRE].

[53] A. Bhardwaj, P. Konar, T. Mandal and S. Sadhukhan, Probing the inert doublet model using jet substructure with a multivariate analysis, Phys. Rev. D 100 (2019) 055040 [arXiv: 1905.04195] [INSPIRE].

[54] R.N. Mohapatra and G. Senjanović, Neutrino Mass and Spontaneous Parity Nonconservation, Phys. Rev. Lett. 44 (1980) 912 [INSPIRE].

[55] J. Schechter and J.W.F. Valle, Neutrino Masses in $\mathrm{SU}(2) \times \mathrm{U}(1)$ Theories, Phys. Rev. D 22 (1980) 2227 [INSPIRE].

[56] E. Gabrielli, M. Heikinheimo, K. Kannike, A. Racioppi, M. Raidal and C. Spethmann, Towards Completing the Standard Model: Vacuum Stability, EWSB and Dark Matter, Phys. Rev. D 89 (2014) 015017 [arXiv: 1309.6632] [INSPIRE].

[57] C.-S. Chen and Y. Tang, Vacuum stability, neutrinos and dark matter, JHEP 04 (2012) 019 [arXiv: 1202.5717] [INSPIRE].

[58] W. Rodejohann and H. Zhang, Impact of massive neutrinos on the Higgs self-coupling and electroweak vacuum stability, JHEP 06 (2012) 022 [arXiv:1203.3825] [INSPIRE].

[59] L. Delle Rose, C. Marzo and A. Urbano, On the stability of the electroweak vacuum in the presence of low-scale seesaw models, JHEP 12 (2015) 050 [arXiv:1506.03360] [INSPIRE]. 
[60] M. Lindner, H.H. Patel and B. Radovčić, Electroweak Absolute, Meta- and Thermal Stability in Neutrino Mass Models, Phys. Rev. D 93 (2016) 073005 [arXiv:1511.06215] [InSPIRE].

[61] J. Chakrabortty, M. Das and S. Mohanty, Constraints on TeV scale Majorana neutrino phenomenology from the Vacuum Stability of the Higgs, Mod. Phys. Lett. A 28 (2013) 1350032 [arXiv: 1207.2027] [INSPIRE].

[62] C. Corianò, L. Delle Rose and C. Marzo, Vacuum Stability in U(1)-Prime Extensions of the Standard Model with TeV Scale Right Handed Neutrinos, Phys. Lett. B 738 (2014) 13 [arXiv: 1407.8539] [INSPIRE].

[63] J.N. Ng and A. de la Puente, Electroweak Vacuum Stability and the Seesaw Mechanism Revisited, Eur. Phys. J. C 76 (2016) 122 [arXiv:1510.00742] [INSPIRE].

[64] C. Bonilla, R.M. Fonseca and J.W.F. Valle, Vacuum stability with spontaneous violation of lepton number, Phys. Lett. B 756 (2016) 345 [arXiv:1506.04031] [INSPIRE].

[65] S. Khan, S. Goswami and S. Roy, Vacuum Stability constraints on the minimal singlet TeV Seesaw Model, Phys. Rev. D 89 (2014) 073021 [arXiv:1212.3694] [InSPIRE].

[66] I. Garg, S. Goswami, K.N. Vishnudath and N. Khan, Electroweak vacuum stability in presence of singlet scalar dark matter in TeV scale seesaw models, Phys. Rev. D 96 (2017) 055020 [arXiv: 1706.08851] [INSPIRE].

[67] N. Chakrabarty, D.K. Ghosh, B. Mukhopadhyaya and I. Saha, Dark matter, neutrino masses and high scale validity of an inert Higgs doublet model, Phys. Rev. D 92 (2015) 015002 [arXiv: 1501.03700] [INSPIRE].

[68] D.K. Ghosh, N. Ghosh, I. Saha and A. Shaw, Revisiting the high-scale validity of the type-II seesaw model with novel LHC signature, Phys. Rev. D 97 (2018) 115022 [arXiv: 1711.06062] [INSPIRE].

[69] E. Ma, Verifiable radiative seesaw mechanism of neutrino mass and dark matter, Phys. Rev. D 73 (2006) 077301 [hep-ph/0601225] [INSPIRE].

[70] K. Kannike, Vacuum Stability Conditions From Copositivity Criteria, Eur. Phys. J. C 72 (2012) 2093 [arXiv: 1205.3781] [INSPIRE].

[71] J. Chakrabortty, P. Konar and T. Mondal, Copositive Criteria and Boundedness of the Scalar Potential, Phys. Rev. D 89 (2014) 095008 [arXiv:1311.5666] [INSPIRE].

[72] J. Horejsi and M. Kladiva, Tree-unitarity bounds for THDM Higgs masses revisited, Eur. Phys. J. C 46 (2006) 81 [hep-ph/0510154] [inSPIRE].

[73] G. Bhattacharyya and D. Das, Scalar sector of two-Higgs-doublet models: A minireview, Pramana 87 (2016) 40 [arXiv: 1507.06424] [INSPIRE].

[74] M.E. Peskin and T. Takeuchi, Estimation of oblique electroweak corrections, Phys. Rev. D 46 (1992) 381 [INSPIRE].

[75] A. Arhrib, R. Benbrik and N. Gaur, $H \rightarrow \gamma \gamma$ in Inert Higgs Doublet Model, Phys. Rev. D 85 (2012) 095021 [arXiv:1201.2644] [InSPIRE].

[76] R. Barbieri, L.J. Hall and V.S. Rychkov, Improved naturalness with a heavy Higgs: An alternative road to LHC physics, Phys. Rev. D 74 (2006) 015007 [hep-ph/0603188] [INSPIRE].

[77] GFitter Group collaboration, The global electroweak fit at NNLO and prospects for the LHC and ILC, Eur. Phys. J. C 74 (2014) 3046 [arXiv:1407.3792] [INSPIRE]. 
[78] E. Lundstrom, M. Gustafsson and J. Edsjo, The Inert Doublet Model and LEP II Limits, Phys. Rev. D 79 (2009) 035013 [arXiv:0810.3924] [InSPIRE].

[79] A. Pierce and J. Thaler, Natural Dark Matter from an Unnatural Higgs Boson and New Colored Particles at the TeV Scale, JHEP 08 (2007) 026 [hep-ph/0703056] [INSPIRE].

[80] Q.-H. Cao, E. Ma and G. Rajasekaran, Observing the Dark Scalar Doublet and its Impact on the Standard-Model Higgs Boson at Colliders, Phys. Rev. D 76 (2007) 095011 [arXiv: 0708.2939] [INSPIRE].

[81] A. Djouadi, The anatomy of electro-weak symmetry breaking. II. The Higgs bosons in the minimal supersymmetric model, Phys. Rept. 459 (2008) 1 [hep-ph/0503173] [INSPIRE].

[82] B. Swiezewska and M. Krawczyk, Diphoton rate in the inert doublet model with a $125 \mathrm{GeV}$ Higgs boson, Phys. Rev. D 88 (2013) 035019 [arXiv:1212.4100] [InSPIRE].

[83] M. Krawczyk, D. Sokolowska, P. Swaczyna and B. Swiezewska, Constraining Inert Dark Matter by $R_{\gamma \gamma}$ and WMAP data, JHEP 09 (2013) 055 [arXiv: 1305.6266] [INSPIRE].

[84] ATLAS collaboration, Measurement of Higgs boson production in the diphoton decay channel in pp collisions at center-of-mass energies of 7 and $8 \mathrm{TeV}$ with the ATLAS detector, Phys. Rev. D 90 (2014) 112015 [arXiv:1408.7084] [INSPIRE].

[85] CMS collaboration, Observation of the Diphoton Decay of the Higgs Boson and Measurement of Its Properties, Eur. Phys. J. C 74 (2014) 3076 [arXiv:1407.0558] [INSPIRE].

[86] S. Vagnozzi et al., Unveiling $\nu$ secrets with cosmological data: neutrino masses and mass hierarchy, Phys. Rev. D 96 (2017) 123503 [arXiv:1701.08172] [INSPIRE].

[87] P.F. de Salas, D.V. Forero, C.A. Ternes, M. Tortola and J.W.F. Valle, Status of neutrino oscillations 2018: $3 \sigma$ hint for normal mass ordering and improved CP sensitivity, Phys. Lett. B 782 (2018) 633 [arXiv:1708.01186] [INSPIRE].

[88] I. Esteban, M.C. Gonzalez-Garcia, M. Maltoni, I. Martinez-Soler and T. Schwetz, Updated fit to three neutrino mixing: exploring the accelerator-reactor complementarity, JHEP 01 (2017) 087 [arXiv: 1611.01514] [INSPIRE].

[89] A. Ilakovac and A. Pilaftsis, Flavor violating charged lepton decays in seesaw-type models, Nucl. Phys. B 437 (1995) 491 [hep-ph/9403398] [INSPIRE].

[90] D. Tommasini, G. Barenboim, J. Bernabeu and C. Jarlskog, Nondecoupling of heavy neutrinos and lepton flavor violation, Nucl. Phys. B 444 (1995) 451 [hep-ph/9503228] [INSPIRE].

[91] D.N. Dinh, A. Ibarra, E. Molinaro and S.T. Petcov, The $\mu-e$ Conversion in Nuclei, $\mu \rightarrow e \gamma, \mu \rightarrow 3 e$ Decays and TeV Scale See-Saw Scenarios of Neutrino Mass Generation, JHEP 08 (2012) 125 [Erratum ibid. 09 (2013) 023] [arXiv: 1205.4671] [INSPIRE].

[92] G. Bambhaniya, P.S. Bhupal Dev, S. Goswami, S. Khan and W. Rodejohann, Naturalness, Vacuum Stability and Leptogenesis in the Minimal Seesaw Model, Phys. Rev. D 95 (2017) 095016 [arXiv: 1611.03827] [INSPIRE].

[93] P. Ghosh, A.K. Saha and A. Sil, Study of Electroweak Vacuum Stability from Extended Higgs Portal of Dark Matter and Neutrinos, Phys. Rev. D 97 (2018) 075034 [arXiv: 1706. 04931] [INSPIRE].

[94] L. Lopez Honorez, E. Nezri, J.F. Oliver and M.H.G. Tytgat, The Inert Doublet Model: An Archetype for Dark Matter, JCAP 02 (2007) 028 [hep-ph/0612275] [INSPIRE]. 
[95] J.M. Alarcon, J. Martin Camalich and J.A. Oller, The chiral representation of the $\pi N$ scattering amplitude and the pion-nucleon sigma term, Phys. Rev. D 85 (2012) 051503 [arXiv:1110.3797] [INSPIRE].

[96] J.M. Alarcon, L.S. Geng, J. Martin Camalich and J.A. Oller, The strangeness content of the nucleon from effective field theory and phenomenology, Phys. Lett. B 730 (2014) 342 [arXiv:1209.2870] [INSPIRE].

[97] J. Herrero-Garcia, A. Scaffidi, M. White and A.G. Williams, On the direct detection of multi-component dark matter: sensitivity studies and parameter estimation, JCAP 11 (2017) 021 [arXiv: 1709.01945] [INSPIRE].

[98] M. Klasen, C.E. Yaguna and J.D. Ruiz-Alvarez, Electroweak corrections to the direct detection cross section of inert Higgs dark matter, Phys. Rev. D 87 (2013) 075025 [arXiv:1302.1657] [INSPIRE].

[99] T. Abe and R. Sato, Quantum corrections to the spin-independent cross section of the inert doublet dark matter, JHEP 03 (2015) 109 [arXiv: 1501.04161] [INSPIRE].

[100] D. Barducci et al., Collider limits on new physics within MicrOMEGAs_4.3, Comput. Phys. Commun. 222 (2018) 327 [arXiv: 1606.03834] [INSPIRE].

[101] A. Semenov, LanHEP: A package for the automatic generation of Feynman rules in field theory. Version 3.0, Comput. Phys. Commun. 180 (2009) 431 [arXiv:0805.0555] [INSPIRE].

[102] E.W. Kolb and M.S. Turner, The Early Universe, Front. Phys. 69 (1990) 1 [InSPIRE].

[103] XENON collaboration, First results on the scalar WIMP-pion coupling, using the XENON1T experiment, Phys. Rev. Lett. 122 (2019) 071301 [arXiv:1811.12482] [INSPIRE].

[104] MAGIC and FERMI-LAT collaborations, Limits to Dark Matter Annihilation Cross-Section from a Combined Analysis of MAGIC and Fermi-LAT Observations of Dwarf Satellite Galaxies, JCAP 02 (2016) 039 [arXiv:1601.06590] [INSPIRE].

[105] M. Gustafsson, E. Lundstrom, L. Bergstrom and J. Edsjo, Significant Gamma Lines from Inert Higgs Dark Matter, Phys. Rev. Lett. 99 (2007) 041301 [astro-ph/0703512] [INSPIRE].

[106] C. Garcia-Cely, M. Gustafsson and A. Ibarra, Probing the Inert Doublet Dark Matter Model with Cherenkov Telescopes, JCAP 02 (2016) 043 [arXiv: 1512.02801] [INSPIRE].

[107] B. Eiteneuer, A. Goudelis and J. Heisig, The inert doublet model in the light of Fermi-LAT gamma-ray data: a global fit analysis, Eur. Phys. J. C 77 (2017) 624 [arXiv:1705.01458] [INSPIRE].

[108] F.S. Queiroz and C.E. Yaguna, The CTA aims at the Inert Doublet Model, JCAP 02 (2016) 038 [arXiv: 1511.05967] [INSPIRE].

[109] C. Garcia-Cely and A. Ibarra, Novel Gamma-ray Spectral Features in the Inert Doublet Model, JCAP 09 (2013) 025 [arXiv: 1306.4681] [INSPIRE].

[110] J.A. Casas and A. Ibarra, Oscillating neutrinos and $\mu \rightarrow e, \gamma$, Nucl. Phys. B 618 (2001) 171 [hep-ph/0103065] [INSPIRE].

[111] G.C. Branco, P.M. Ferreira, L. Lavoura, M.N. Rebelo, M. Sher and J.P. Silva, Theory and phenomenology of two-Higgs-doublet models, Phys. Rept. 516 (2012) 1 [arXiv:1106.0034] [INSPIRE]. 
[112] Yu.F. Pirogov and O.V. Zenin, Two loop renormalization group restrictions on the standard model and the fourth chiral family, Eur. Phys. J. C 10 (1999) 629 [hep-ph/9808396] [INSPIRE].

[113] F. Staub, SARAH 4: A tool for (not only SUSY) model builders, Comput. Phys. Commun. 185 (2014) 1773 [arXiv: 1309.7223] [INSPIRE].

[114] W.-l. Guo, Z.-z. Xing and S. Zhou, Neutrino Masses, Lepton Flavor Mixing and Leptogenesis in the Minimal Seesaw Model, Int. J. Mod. Phys. E 16 (2007) 1 [hep-ph/0612033] [INSPIRE].

[115] N. Khan and S. Rakshit, Constraints on inert dark matter from the metastability of the electroweak vacuum, Phys. Rev. D 92 (2015) 055006 [arXiv: 1503.03085] [INSPIRE].

[116] J. Kalinowski, W. Kotlarski, T. Robens, D. Sokolowska and A.F. Zarnecki, Benchmarking the Inert Doublet Model for $e^{+} e^{-}$colliders, JHEP 12 (2018) 081 [arXiv:1809.07712] [INSPIRE].

[117] A. Alloul, N.D. Christensen, C. Degrande, C. Duhr and B. Fuks, FeynRules 2.0 - A complete toolbox for tree-level phenomenology, Comput. Phys. Commun. 185 (2014) 2250 [arXiv: 1310.1921] [INSPIRE].

[118] J. Alwall, M. Herquet, F. Maltoni, O. Mattelaer and T. Stelzer, MadGraph 5: Going Beyond, JHEP 06 (2011) 128 [arXiv:1106.0522] [INSPIRE].

[119] T. Sjöstrand, S. Mrenna and P.Z. Skands, PYTHIA 6.4 Physics and Manual, JHEP 05 (2006) 026 [hep-ph/0603175] [INSPIRE].

[120] H1And For the ZEUS collaboration, Parton Distribution Functions, in Proceedings, 31st International Conference on Physics in collisions (PIC 2011): Vancouver, Canada, August 28 - September 1, 2011, arXiv:1111.5452 [INSPIRE].

[121] J. Alwall et al., The automated computation of tree-level and next-to-leading order differential cross sections and their matching to parton shower simulations, JHEP $\mathbf{0 7}$ (2014) 079 [arXiv: 1405.0301] [INSPIRE].

[122] B.W. Lee, C. Quigg and H.B. Thacker, Weak Interactions at Very High-Energies: The Role of the Higgs Boson Mass, Phys. Rev. D 16 (1977) 1519 [inSPIRE]. 